\title{
Spectral remote sensing for onshore seepage characterization: A critical overview
}

\author{
Saeid Asadzadeh ${ }^{1^{*}}$ \\ saeid@ige.unicamp.br \\ Carlos Roberto de Souza Filho ${ }^{1}$ \\ beto@ige.unicamp.br \\ ${ }^{1}$ Geosciences Institute, University of Campinas-UNICAMP, PO Box 6152, 13083-970 Campinas, SP, Brazil
}

\begin{abstract}
In this article, we overview the application of spectral remote sensing data collected by multi-, and hyperspectral instruments in the visible-near infrared (VNIR), short-wave infrared (SWIR), and longwave infrared (LWIR) wavelengths for characterization of seepage systems as an exploration indicator of subsurface hydrocarbon (HC) accumulations. Two seepage systems namely macro-, and microseepage are recognized. A macroseepage is defined as visible indications of oil and gas on the surface and in the air detectable directly by a remote sensing approach. A microseepage is defined as invisible traces of light HCS in soils and sediments that are detectable by its secondary footprints in the strata, hence an indirect remote sensing target. Based on these broad categories, firstly, a comprehensive set of well-described and reliable remote sensing case studies available in the literature are thoroughly reviewed and then systematically assessed as regards the methodological shortcomings and scantiness in data gathering, processing, and interpretation. The work subsequently attempts to go through seminal papers published on microseepage concept and interrelated geochemical and geophysical techniques, exhumed HC reservoirs, lab-based spectroscopic analysis of petroleum and other related disciplines from a remote sensing standpoint. The aim is to enrich the discussion and highlight the still unexplored capabilities of this technique in accomplishing exploration objectives using the concept of seepage system. Aspects of seepage phenomenon in environmental pollution and uncertainties associated with their role in global warming are also underlined. This work benefits from illustrative products generated over two study areas located in the Ventura Basin, State of California, USA and the Tucano Basin, State of Bahia, Brazil known to host distinctive macro-, and microseepage systems, respectively. In conclusion, we recommend further research over a diverse range of seepage systems and advocate for a mature conceptual model for microseepage phenomenon.
\end{abstract}

Keywords: seepage; microseepage system; hydrocarbon; gas-plume; onshore; spectroscopy; remote sensing; exploration; environment; methane budget.

\section{Introduction}

A large portion of hydrocarbon ( $\mathrm{HC}$ ) traps is not perfectly sealed and thus, their accumulations leak to the surface over time. When the surface manifestation of oil and gas is clearly visible by naked eye, it is termed as macroseepage, whereas the traces of invisible light HCs in near-surface soils and sedimentary rocks (sediments henceforth), which are only detectable by analytical methods and careful geochemical sampling, is called microseepage (Horvitz, 1985; Tedesco, 1995). Historically, seepage and HC accumulations have been tied together and, as a result, a large number of the world's oil and gas fields have been explored by drilling in the immediate area of a seep (Hunt, 1996; Yergin, 1992). In modern exploration programs, macroseeps are typically regarded as direct clues for the existence of mature source rock(s) and a compelling evidence for the formation of a petroleum system in a given sedimentary basin (Magoon and Beaumont, 
1999; Schumacher, 2010), whereas microseeps, which are argued to occur in a near vertical fashion over an accumulation, are employed as a targeting tool for petroleum exploration.

Recent investigations have also revealed that seeps are a potent source of methane (with ethane and propane) greenhouse gasses to the atmosphere. It has been estimated that in the natural methane budget, seeps are the second most important source of emissions after wetlands. The estimates also reveal that onshore seepages are a more significant emitter of $\mathrm{CH}_{4}$ than their offshore counterparts (Etiope, 2015; Etiope and Ciccioli, 2009; Etiope and Klusman, 2010; Etiope et al., 2008).

Over the years, a diverse range of techniques, including remote sensing, has been employed for seepage detection. The remote sensing approach holds a great promise for this aim because it is a fast and costeffective tool applicable to different operational scales for both direct and indirect seepage mapping. In the marine environment, this technology already provides a variety of sensing methods comprising laser florescence, synthetic aperture radar (SAR), and thermal infrared, to name a few (Leifer et al., 2012a). Terrestrial seepage detection, however, has relied heavily on spectral remote sensing collected by multi-, and hyperspectral instruments in the visible-near infrared (VNIR; 0.4-1.0 $\mu \mathrm{m}$ ), the short-wave infrared (SWIR; 1.0-2.5 $\mu \mathrm{m}$ ), and very occasionally in the longwave infrared (LWIR; 8-14 $\mu \mathrm{m}$ ) wavelengths. This methodology has been employed to detect oil and gas seeps in a direct manner and the footprints of $\mathrm{HC}$ leakage in sedimentary units indirectly.

Whereas direct detection of $\mathrm{HCs}$ is a new research topic conducted mainly by airborne imaging spectroscopy, the indirect HC prospecting commenced with the launch of the first Landsat-MSS multispectral scanner (ERTS) in the 1970s (Simpson, 1978). The advent of the hazy anomaly over some productive/prospective fields was simultaneous to a renewed interest in microseepage concept (Donovan, 1974) and thereupon led to several research studies, including a NASA-Geosat joint project, to evaluate the use of satellite technology for HC prospecting (Abrams et al., 1985). Since then, sporadic case studies have appeared in the literature demonstrating the potentials and premises of this approach for oil and gas exploration (see van Der Meer (2002) and Yang (2000) and references therein) that in comparison to extensively studied offshore cases are almost negligible (Fingas and Brown, 2014; Leifer et al., 2012a).

Despite all the merits of spectral remote sensing, the technique does not yet hold a good place amongst unconventional exploration methods for oil and gas resources nor is acknowledged as a mature exploration tool by explorationists. In this article, we set out to discover the full potential of this state-of-the-art technology for seepage characterization and understand the reasons for which the approach is somehow underutilized by the petroleum industry. To fulfill this aim, we provide a systematic and critical overview of the subject based on well-described and reliable remote sensing case studies reported in the literature and thereupon attempt to address methodological shortcomings and inadequacies in data gathering, processing, and interpretations. In addition, we go through the seminal papers published on microseepage theory and interrelated geochemical and geophysical techniques from a remote sensing standpoint to enrich the discussion and highlight the unexplored capabilities of the technique in accomplishing the objectives of exploration sector. We also attempt to engage the attention of the community to useful case studies conducted over exhumed $\mathrm{HC}$ reservoirs as an analogy to depleted traps. In the case of direct seepage detection, we review the few present case studies and contrast their achievements to the findings of interrelated disciplines to underline a wide range of possibilities from spectral products. Lastly, we accentuate the uncertainties about the role of natural seepage in greenhouse gas emission and the possible ways to resolve it. This paper benefits from illustrative products generated over two case studies located in 
the Ventura Basin, California, USA and the Tucano Basin in Bahia state, Brazil known to host distinctive macro-, and microseepage systems, respectively.

\section{Petroleum seepage}

Surface manifestation of oil and gas can be divided into two broad categories namely macro- and microseepage (Fig. 1). Macroseepage is the surface expression of a leakage pathway, typically related to tectonic discontinuities, along which natural liquid or gaseous HCs is (has been) flowing from a subsurface source (Clarke and Cleverly, 1991; Link, 1952; Macgregor, 1993). Microseepage, in contrast, refers to the slow, invisible, but pervasive migration of light alkanes $\left(C_{1}-C_{5}\right)$ and volatiles from accumulation to the surface (Etiope, 2015; Price, 1986; Schumacher, 1999). A microseepage is not related to faults but it can be enhanced by the presence of faults and large fractures (Richers et al., 1982). Here we emphasize that this division is not certain and in reality, there is a seepage continuum from minute microseeps to visible macroseeps (Etiope, 2015; Schumacher, 1999). Through this paper, 'seeps' and 'seepage' are used as generic terms to refer to both of the seepage systems. Furthermore, 'petroleum' and 'hydrocarbon' terms are interchangeably used to denote oil and gas, and any similar organic (natural/artificial) compounds, however, 'oil' is exclusively used to refer to 'crude oil'.

\subsection{Macroseepage systems}

Macroseeps have been classified according to their geological context, underlying tectonic regime, activity manner, migration pathway, leakage rate (intensity), occurrence environment, and associated alteration patterns (Abrams, 2005; Clarke and Cleverly, 1991; Link, 1952; Macgregor, 1993; Thrasher et al., 1996). However, for the purpose of this paper, we only distinguish them to be either oil or gas seepage, respectively dominated by liquid and gaseous phases. In terms of activity, flowing (active) oil seeps shall be differentiated from paleo-seeps (impregnations) wherein no evidence for their replenishment exists (Macgregor, 1993). Depending on their leaking state, oil seeps can incorporate liquid and solid petroleum, tar, and bitumen.

Some macroseeps have a diffused lateral flow in near-surface fractures thereupon trigger the formation of local alterations around the principle oil/gas seep (Fig. 1). To discriminate such alterations from those associated with a microseepage system (section 2-2), a new term called miniseepage has been introduced in the literature (Etiope, 2015). Here we adopt the same terminology to discern between the two seepagerelated alterations, but since the final mineral assemblages are rather identical, we discuss them under the same section. Some studies have demonstrated that around 30\% of the known macroseeps are associated with surficial anomalies of one type or another (Clarke and Cleverly, 1991), which based on the given definition, can indicate the overall proportion of miniseeps.

\subsection{Microseepage systems}

The long-term leakage of HCs in a microseepage system normally induces an array of diagenetic physiochemical and mineralogical transformations in the chimney column above $\mathrm{HC}$ accumulations (Fig. 1). The activity and by-products of bacteria and other microbes are believed to change the $\mathrm{pH}$-Eh of the overlying stratigraphic column and initiate a series of diagenetic changes including (i) biological (microbial/geobotanical) anomalies; (ii) mineralogical alterations; (iii) electrochemical changes and resistivity abnormalities; (iv) magnetic iron oxides and sulfides; and (v) radiation anomalies (Etiope and Martinelli, 2002; Price, 1986; Saunders et al., 1999; Schumacher, 1996; Tedesco, 1995; Warren, 2012) (Fig. 1). 
The mineralogical changes of the classic microseepage model, which is the focus of this review paper, comprises the following patterns (Fig. 1):

Carbonate precipitation: carbonate cement are quite common in microseepage systems. The diagenetic carbonate species detected so far is very diverse and ranges from calcite and ferroan calcite to dolomite, ankerite, siderite, rhodochrosite, and aragonite; though calcite by far is the dominant secondary carbonates (Al Shaieb et al., 1994; Saunders et al., 1999; Schumacher, 1996). The extent of carbonate cementation is not commonly known, albeit, in Cement Field, it was reported to cover an area of about $3.0 \mathrm{~km}^{2}$ in total (Kirkland et al., 1995). Several studies have indicated that carbon of carbonates can be originated from HC oxidation (Donovan et al., 1974), however, it is not always the case and carbon can originate from other sources like meteoric or Interstitial water (Beitler et al., 2005). The ultimate species of carbonate in diagenetic facies is shown to be related to the concentrations of $\mathrm{Fe}, \mathrm{Mg}$, and $\mathrm{Ca}$ ion in the system (Al Shaieb et al., 1994).

Bleached red-beds: the discoloration, or bleaching, occurs due to the removal of ferric iron oxides (i.e. hematite) coating from red-beds. This phenomenon, which is chiefly controlled by the fabric of the host rock, is responsible for most of the visual color changes in the chimney column (Donovan, 1974; Schumacher, 1996). The iron discoloration can be due to bleaching, which involves chemical reduction and removal of iron from the system, or enrichment, which is the secondary precipitation of additional patchy iron oxide cement in the strata (Nielson et al., 2014) (see also Fig. 6e). The bleached ferric iron can ultimately take the following forms: (i) reduction into ferrous species; (ii) partial/total removal from the system; and (iii) re-precipitation as iron oxide concretions (Parry et al., 2009). Whereas some ferrous irons are likely to be carried away from a system, a significant proportion of it can be introduced into lower strata by descending meteoric water. Subsequent oxidation of these iron-rich horizons can bring about ironstones and ferricrete features (Nielson et al., 2014).

Clay formation: clay formation/transformation is mostly related to slightly acidic conditions in the chimney column. Kaolinite, which by far is the prevalent clay in microseepage-induced alterations, is believed to form after the alteration of feldspars or the conversion of then unstable illitic/smectitic clays (see Fig. 6d). This process can liberate potassium (K) from clays, and thus lead to low gamma-ray radiations above HC deposits (Saunders et al., 1993a). The conductive anomalies noted over some chimney column are attributed to the presence of dioctahedral and trioctahedral clays in the system (Warren, 2012).

Sulfide formation: pyrite constitutes the dominant sulfide mineral, though other sulfides like pyrrhotite, marcasite, galena, greigite, and native sulfur are locally abundant (Al Shaieb et al., 1994; Schumacher, 1996). The abundance of pyritic zone depends on several parameters including the abundance of sulfur (S) in the environment. The sulfur itself has been postulated to be originated either from $\mathrm{H}_{2} \mathrm{~S}$ associated with seeping HCs or from sulfates after bacterial metabolism (Lilburn and Zuhair, 1984). The native sulfur can be the result of hydrogen sulfide oxidation or the reaction of non-methane HCs with anhydrite (Worden et al., 1997). Sulfidization is a rather uncommon process in alteration facies.

The occurrence of pyrrhotite and greigite along with maghemite and magnetite are known to be responsible for anomalous magnetic susceptibility above many chimney columns (Schumacher, 1996). Due to the role of magnetotactic bacteria in forming such magnetic bodies, some have postulated that magnetic anomalies shall approximate the 'fossil' bacterial activities within a system (Foote, 2013; Saunders et al., 1999). 

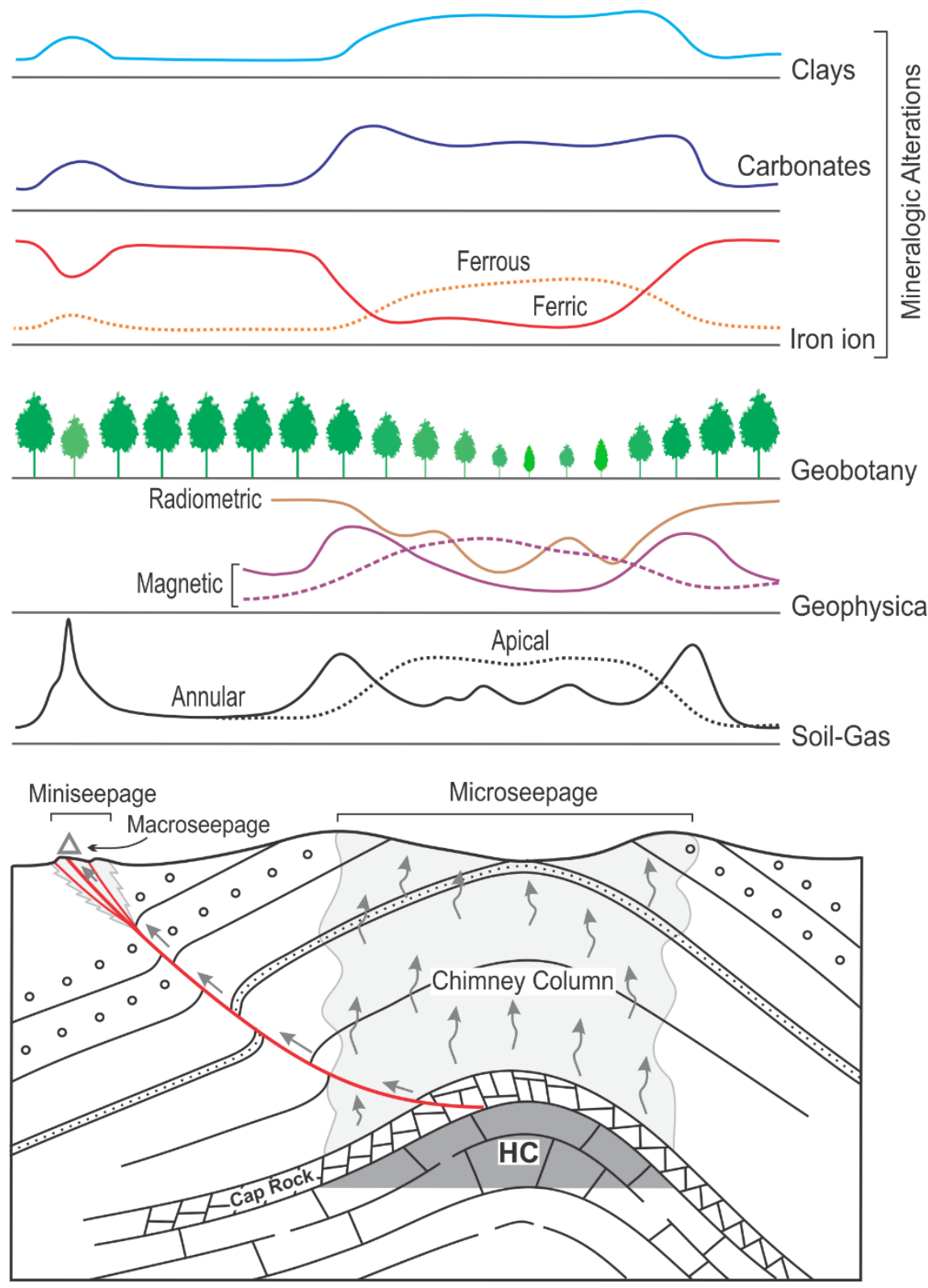

Fig. 1. Schematic representation of microseepage, miniseepage, and macroseepage concepts and associated HC-induced anomalies (adapted and modified partly after (Duchscherer, 1982; Eventov, 2000; Saunders et al., 1993b; Saunders et al., 1999; Schumacher, 1996; van der Werff, 2006)). The HC accumulation and induced alterations are shown by dark-gray and gray shades, respectively. The anomalous signatures encompass geochemical (soil-gas), geophysical, and remote sensing (geobotany and mineralogical alterations) sub-groups.

For decades, the microseepage theory has been controversial among explorationists. However, now there are several (empirical and theoretical) lines of evidence that support this postulation: (i) the $C_{1}$ to $C_{5}$ composition of the migrating gas that is similar to the gas phase of the pool; (ii) the isotopic content of the seeping HCs that matches that of the underlying reservoir; (iii) the dynamic character of the leakage in responding to reservoir depletion or recharge; (iv) the close conformity of the anomalies with the surface projection of HC reservoir; (v) the bloom of HC-degrading bacteria over accumulations; (vi) the direct evidence provided by well-cutting measurements; and (vii) the gas chimneys imaged by high resolution 3D seismic data (Connolly et al., 2013; Donovan, 1974; Duchscherer, 1980; Foote, 2013; Holysh and Toth, 1996; Horvitz, 1980; Horvitz, 1985; Jones and Drozd, 1983; Price, 1986; Tedesco, 1995; Thompson et al., 1994). The concept of chimney cube, which highlights the chaotic behavior of seismic characters due to gas migration (Aminzadeh et al., 2001), not only is providing a new imaging indicator for HC leakage, but also is bridging 
the gap between seismic and geochemical surveys and likely is going to end the skepticism and debate on the concept.

The migration processes of gaseous HCs is not well understood, but some computer simulations have shown that pressure-driven continuous gas-phase flow through fractures or the transition from bubble regimes to continuous phase flow can be the plausible mechanism (Brown, 2000; Etiope and Martinelli, 2002). The proposed mechanism was demonstrated, for example, to successfully explain the rapid emerge of gaseous anomalies over gas storage fields.

\subsection{Macro- versus microseepage}

Macroseeps typically migrate laterally for a few to dozens of kilometers through major tectonic discontinuities (Link, 1952; Macgregor, 1993; Thrasher et al., 1996) and thus occur distal to accumulations, whereas microseeps migrate almost vertically and hence are proximal to the pool. Different aspects of macro-, and microseepage systems are compared in Table 1.

Table 1. A comparison between different aspects of micro- and macroseepage systems.

\begin{tabular}{|l|l|l|}
\hline \multicolumn{1}{|c|}{ Parameter } & \multicolumn{1}{|c|}{ Microseepage } & \multicolumn{1}{c|}{ Macroseepage } \\
\hline $\begin{array}{l}\text { Direct detection } \\
\text { approach }\end{array}$ & Analytical & Visual \\
\hline Agent (HC content) & $\begin{array}{l}\text { Light HCs }\left(\mathrm{C}_{1}-\mathrm{C}_{5} \text { ), }\right. \\
\text { volatiles (aromatics) }\end{array}$ & $\begin{array}{l}\text { Heavy/light crude oil, } \\
\text { volatiles, gaseous HCs }\end{array}$ \\
\hline Migration mechanism & $\begin{array}{l}\text { Continuous gas-phase } \\
\text { flow in micro-fractures }\end{array}$ & $\begin{array}{l}\text { Effusion through } \\
\text { tectonic discontinuities }\end{array}$ \\
\hline $\begin{array}{l}\text { Abundance in } \\
\text { petroliferous areas }\end{array}$ & More than 80\% & Around 20\% \\
\hline Migration fashion & Mostly vertical & Mostly lateral \\
\hline Alteration & $\begin{array}{l}\text { Yes, extensive } \\
\text { (chimney column) }\end{array}$ & $\begin{array}{l}\text { Yes/No, limited } \\
\text { (miniseep) }\end{array}$ \\
\hline Spatial (areal) extent & Pervasive halo & Point targets \\
\hline Associated trap & Structural, stratigraphic & Mainly structural \\
\hline Flux (mg/m ${ }^{2} / \mathrm{d}$ ) & Tens & Hundreds to thousands \\
\hline Exploration significance & Indirect & Direct \\
\hline Types of activity & Active vs fossil (inactive) & $\begin{array}{l}\text { Active vs passive } \\
\text { Flowing vs } \\
\text { impregnations }\end{array}$ \\
\hline Targeting potential & Yes & No \\
\hline $\begin{array}{l}\text { Relationship to } \\
\text { accumulation }\end{array}$ & Simple, proximal & Complex, distal \\
\hline
\end{tabular}

Historically, macroseeps have been the stimulus for early exploration drilling, and statistics reveals that almost all of the world's major oil fields in the beginning of $20^{\text {th }}$ century have been associated with seeps. By 1950s, at least half of the proven reserves were discovered by drilling near macroseeps, whereas by the end of the last century, this proportion plummeted to around 20\% (Clarke and Cleverly, 1991; Hunt, 1996; Link, 1952; Macgregor, 1993). On the other hand, more than $80 \%$ of the $\mathrm{HC}$ reservoirs are associated with microseeps of different manifestations (Schumacher, 2010), and unlike macroseeps that are general basinal scale prospecting indicators (Macgregor, 1993), microseeps have proved to be a targeting tool for subsurface $\mathrm{HC}$ accumulations. For example, with surface geochemical methods, $70-82 \%$ of subsequently commercial discoveries, and about $90 \%$ of the eventually dry holes have been reliably predicted, which are respectively equivalent to $11 \%$ false-negative and $18 \%$ false-positive proportions (Schumacher, 2010). Other statistics has 
revealed that $76 \%$ of the prospects with soil gas anomaly above them have ended up as commercial discoveries (Schumacher, 2000). Further discussion on this topic is provided in section 6-2-8.

Even though many onshore macroseeps have already been accounted for and their attributes are reflected in compiled global databases (Clarke and Cleverly, 1991; Etiope, 2009), the distribution and varieties of onshore microseeps have remained largely unknown. The great number of documented seeps $(>10,000$ entities) indeed implies that there is a staggering potential for microseeps yet to be detected. Fortunately, the spectral remote sensing is quite capable of closing the gap in our knowledge of petroleum seepage systems. Typically, the higher flux of macroseeps (several to hundreds of $\mathrm{kgd}^{-1}$ ) permits remote sensing techniques to 'directly' target them using the diagnostic spectral signatures of escaping HCs (solid/liquid/gas), whereas the existence of microseeps (with fluxes in the order of a few to tens of $\mathrm{mgm}^{-2} \mathrm{~d}^{-1}$ ) are usually inferred 'indirectly' using the alteration mineralogy induced by microseepage systems.

In the literature, there is no account on the sizes of macroseepage as potential targets for remote sensing. However, our experience indicates that the majority of them constitute tiny targets of a few meters (See for instance Fig. 5e-5f). In contrast, microseepage-induced changes are proportional to accumulation extent and thus set larger targets.

\section{Remote sensing of macroseepages}

\subsection{Spectroscopy of petroleum}

A number of structural bonds in petroleum including $\mathrm{CH}, \mathrm{CH}_{2}, \mathrm{CH}_{3}$, and $\mathrm{C}=\mathrm{C}$ give rise to several fundamental absorption bands between 3000-9000 nm wavelengths (Cloutis, 1989; Coates, 2006; Lammoglia and Souza Filho, 2011) (Table 2). Within the VNIR-SWIR wavelengths, petroleum also retains a series of absorption bands due to overtones and combinations of noted stretching fundamentals. The most notable feature in the VNIR-SWIR range includes a triplet between 1700-1750 nm and a doublet between 2290-2360 nm (Fig. 2 and Fig. 5d). Occasionally, subtle features around 1120-1230 and 1350-1550 nm can be present in the spectra of some lighter oils (Fig. 2).

To promote our understanding of the absorbing bands involved in each feature, we measured a light oil sample (API=43.2) with an ASD FieldSpec-4 spectrometer between 0.35-2.5 $\mu \mathrm{m}$ (the details of the sample and measurement protocol is provided in Asadzadeh (2016a)). The resulting spectrum was then analyzed by means of the Modified Gaussian Model (MGM) deconvolution technique (Sunshine et al., 1990). Results indicated that around 20 individual absorption bands are resolvable (Fig. 2) with each of the noted features being decomposed into multiple overlapping bands. A case in point is the prominent features at 2300 and $1700 \mathrm{~nm}$ that are respectively comprised of seven and five overlapping bands of varying width and intensities (Fig. 2).

Based on the fundamental absorption features reported in Table 2 and the physical basis of spectroscopy, we attempted to calculate the position of resolved absorption bands by considering the overtones and combinations of the fundamentals bands along with the combination of overtones, the combination of overtones and fundamentals, and the overtone of combinations. The findings are reported in Table 3 and illustrated in Fig. 2. Seemingly, there is a close match between the observed bands and the results of mathematical calculations. Moreover, each individual band usually corresponds to a collection of theoretical possibilities (Table 3), indicating the complexity of the spectra and the difficulty of such interpretations. In a highly complicated compound like petroleum, a sheer number of absorbing bands can 
develop in the SWIR range, albeit the majority of them are averaged out due to overlapping and only the more pronounced bands or the contributors with constructive bands are left out as absorption features in the spectrum (Clark et al., 2009; Cloutis, 1989). This process is responsible for an intense backdrop absorption and a low overall reflectance $(<20 \%)$ of petroleum. Based on band assignment outcomes, we roughly divided the SWIR wavelength into three sub-ranges: (i) between 1050-1350 nm in which first/second overtones are dominant; (ii) between 1350-2100 in which first overtones and combinations are dominant; and (iii) beyond $2100 \mathrm{~nm}$ in which the combinations are the dominant absorption mechanism (Fig. 2).

Table 2. The main fundamental absorption bands of organic compounds and HCs (Modified after (Cloutis, 1989; Coates, 2006)).

\begin{tabular}{|c|l|l|l|}
\hline \multirow{2}{*}{ Band } & \multicolumn{2}{|c|}{$\begin{array}{c}\text { Fundamental } \\
\text { Wavelength/wavenumber }\end{array}$} & \multirow{2}{*}{ Fundamental group/assignment } \\
\cline { 2 - 3 } & \multicolumn{1}{|c|}{$\boldsymbol{\mu m}$} & \multicolumn{1}{|c|}{$\mathbf{c m}^{-1}$} & \\
\hline a & 2.81 & 3550 & Hydroxyl O-H stretch \\
\hline b & $3.28-3.30$ & $3030-3050$ & Alkene, aromatic (aryl) C-H stretch \\
\hline c & 3.38 & 2960 & Asymmetric $\mathrm{CH}_{3}$ (methyl) stretch \\
\hline d & 3.42 & 2925 & Asymmetric $\mathrm{CH}_{2}$ (methylene) stretch \\
\hline e & 3.48 & 2870 & Symmetric $\mathrm{CH}_{3}$ stretch \\
\hline f & 3.50 & 2855 & Symmetric $\mathrm{CH}_{2}$ stretch \\
\hline g & $5.78-5.88$ & $1700-1730$ & Carbonyl-carboxyl C=O stretch \\
\hline h & $6.06-6.25$ & $1600-1650$ & Aromatic, alkenyl carbon $(\mathrm{C}=\mathrm{C})$ stretch \\
\hline i & $6.82-6.90$ & $1450-1465$ & Asymmetric $\mathrm{CH}_{3}, \mathrm{CH}_{2}$ bend \\
\hline j & 7.27 & 1375 & Symmetric $\mathrm{CH}_{3}$ bend \\
\hline k & 9.71 & 1030 & Aromatic $\mathrm{C}-\mathrm{H}_{\text {bend }}$ \\
\hline
\end{tabular}

The pronounced absorption feature occurring in the VNIR was modeled by a single Gaussian band and ascribed to the pi $(\pi$ ) electronic bonding between carbon atoms (Fig. 2). Typically, the minimum of this broad absorption shifts towards longer wavelength as the average number of aromatic rings per molecule (i.e. the aromatization type and degree) and the complexity of HC molecules increases (Cloutis, 1989; Cloutis, 1990). In other words, as oil becomes heavier in terms of API gravity, the absorption tends to become broader while its minimum moves towards longer wavelengths (see Fig. 3 in (Asadzadeh and Souza Filho, 2016a)). As a result, the stretching wing of this broad absorption that reaches up to $1500 \mathrm{~nm}$ and beyond can potentially mask the subtle (and rather weaker) features occurring at 1200 and $1400 \mathrm{~nm}$. We should emphasize that except the feature centered at $1400 \mathrm{~nm}$ that coincides with atmospheric water vapor, other features can be potentially used for remote sensing $\mathrm{HC}$ detection.

Table 3. Calculated wavelength positions of HCs arising from overtones and combinations of the fundamental absorption bands listed in Table 2. The numbers "2", "3", and "+" symbols denote the 1st and $2^{\text {nd }}$ overtones and combinations of relevant bands, respectively.

\begin{tabular}{|c|c|c|c|c|c|}
\hline $\begin{array}{c}\text { Overtone/combination } \\
\text { band }\end{array}$ & $\begin{array}{c}\text { Wavelength } \\
(\mu \mathrm{m})\end{array}$ & $\begin{array}{c}\text { Observed } \\
(\mu \mathrm{m})\end{array}$ & $\begin{array}{c}\text { Overtone/combination } \\
\text { band }\end{array}$ & $\begin{array}{c}\text { Wavelength } \\
(\mu \mathrm{m})\end{array}$ & $\begin{array}{c}\text { Observed } \\
(\mu \mathrm{m})\end{array}$ \\
\hline $3 \mathrm{e}$ & 1.16 & 1.16 & $2 f$ & 1.75 & \multirow{2}{*}{1.76} \\
\hline $3 f, 2(f+j)$ & 1.17 & 1.19 & $2 j+d$ & 1.76 & \\
\hline $2 k+2 b$ & 1.22 & 1.21 & $2 g+2 k$ & 1.81 & 1.81 \\
\hline $2 d+i$ & 1.37 & \multirow{2}{*}{1.39} & $3 g$ & 1.93 & 1.94 \\
\hline $2 d+j$ & 1.38 & & $d+h$ & 2.18 & \multirow{2}{*}{2.19} \\
\hline $2 a, 2 b+k$ & 1.40 & 1.41 & $c+h, f+g$ & 2.19 & \\
\hline $2 d+k$ & 1.45 & 1.45 & $e+h, f+h$ & 2.23 & 2.23 \\
\hline$a+c$ & 1.68 & \multirow{2}{*}{1.70} & $c+i$ & 2.27 & 2.28 \\
\hline $2 c, b+e$ & 1.69 & & $c+j, e+i$ & 2.31 & 2.31 \\
\hline
\end{tabular}




\begin{tabular}{|c|c|c|c|c|c|}
\hline$b+f, c+d$ & 1.70 & & $d+j, 2 i+j$ & 2.32 & \\
\hline $2 d, c+e$ & 1.71 & & $e+j$ & 2.35 & \multirow{2}{*}{2.35} \\
\hline$c+f, 2 i+d$ & 1.72 & \multirow{3}{*}{1.73} & $f+j$ & 2.36 & \\
\hline$d+f$ & 1.73 & & $2 j+i$ & 2.38 & 2.39 \\
\hline $2 \mathrm{e}$ & 1.74 & & $b+k$ & 2.46 & 2.47 \\
\hline
\end{tabular}

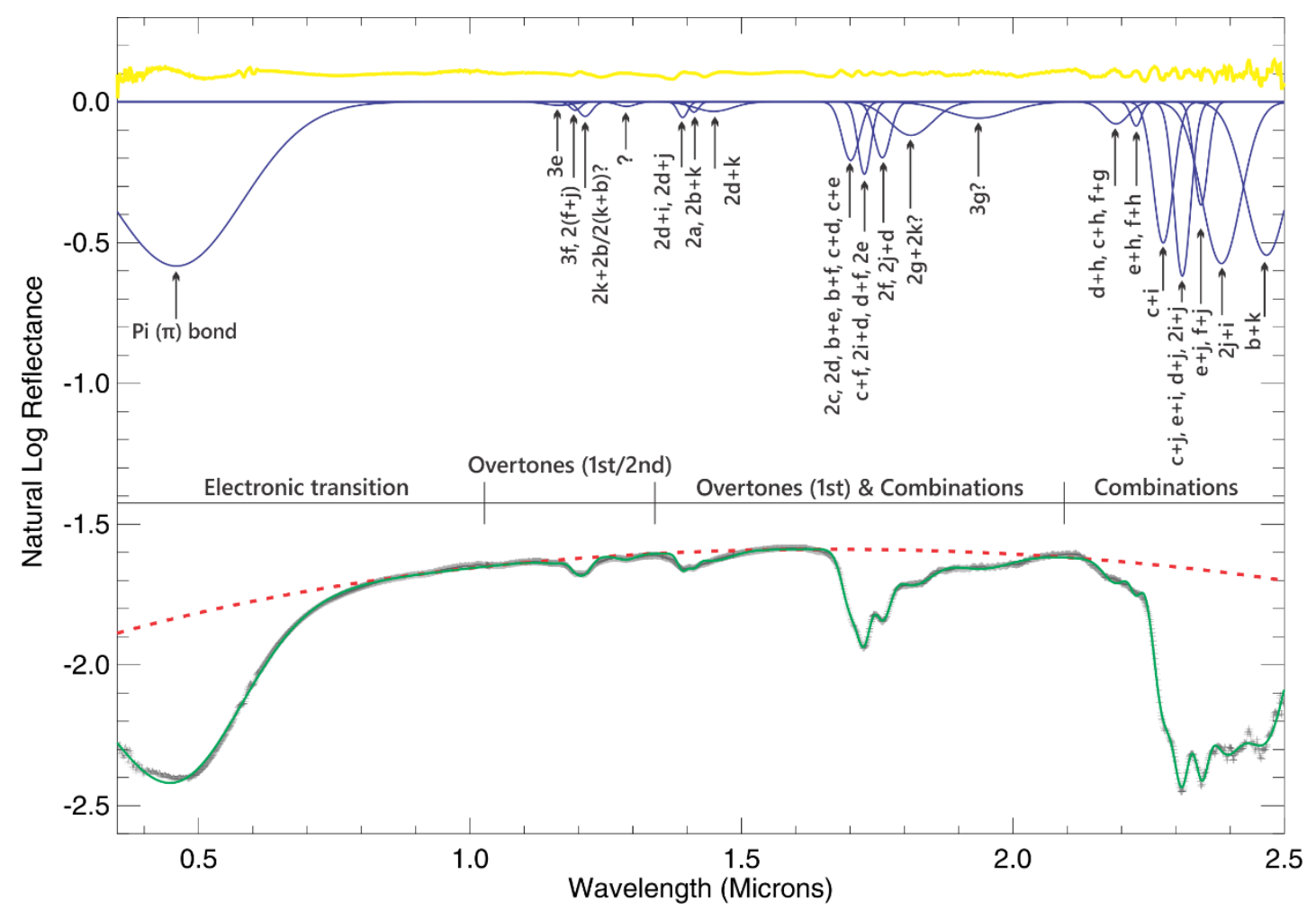

Fig. 2. Spectral deconvolution of a light $\mathrm{HC}$ spectrum using the MGM deconvolution technique. Plausible absorption mechanisms are provided for each of the resolved bands in the figure. The details of the band assignment are indicated in Table 3. The dashed red line represents the continuum modeled as a second-order polynomial. The solid yellow line illustrates the fitting error. The solid blue lines are the individual Gaussian bands. The original spectrum in gray is superimposed by the modeled spectrum (the combination of resolved bands) and shown in green.

Due to similar processes, methane and other lightweight alkane molecules $\left(C_{1}-C_{10+}\right)$ show distinctive absorption features in the SWIR as well as in the mid-infrared (MIR) and LWIR (e.g. between 7-8 $\mu \mathrm{m}$ ) ranges. Reflectance spectroscopy of alkane series has been comprehensively covered in (Clark et al., 2009) (see also section 3-3).

\subsection{An overview of the case studies}

Despite the merit of spectral remote sensing in macroseepage detection, only a handful of case studies are available in the literature on the subject that are summarized in Table 4. The first study of this kind was facilitated by the Geosat Committee in 1998, wherein a Probe-1 hyperspectral scanner was flown over some well-known seeps in the Ventura basin, California. The study demonstrated that the seeping oil is spectrally mappable by a far-range system provided that the sensor has enough spatial and spectral resolution (Ellis et al., 2001). Although this study merely focused on the $2300 \mathrm{~nm}$ features, later investigations employed the feature centered at $1700 \mathrm{~nm}$ for $\mathrm{HC}$ detection using some simulated oil contaminations (Table 4). On the basis of that experiment, a 'hydrocarbon index' was proposed for $\mathrm{HC}$ targeting using the mere radiance dataset (Hörig et al., 2001; Kühn et al., 2004). In 2013, the oil seeps at the Ventura basin were surveyed once 
more using a bundle of hyperspectral imaging systems in the VNIR-SWIR-LWIR ranges. The conducted experiment with the SWIR subset indicated that a systematic remote sensing survey in a mature basin not only can map the extent of already known seeps accurately but also can lead to new seepage discoveries (Prelat et al., 2013). Correspondingly, in the case study reported in Fig. 5, the authors were unaware of the existence of Seep-4 beforehand and it was recognized just after spectral analysis (see Fig. 5f).

Table 4. Summary of the case studies directed towards the detection of petroleum macroseeps (and onshore oil contaminations) using spectral remote sensing techniques. The detection limit is defined as the smallest dimension of a target detectable spectrally. The acronyms used in the table are BR: Band ratioing, RBD: relative absorption band-depth, DT: decision tree, SAM: spectral angle mapper, MF: matched filtering, MTMF: mixture-tuned matched filtering, GSD: ground sampling distance, HSI: Hyperspectral imaging.

\begin{tabular}{|c|c|c|c|c|c|c|c|c|c|}
\hline \multirow[b]{2}{*}{ Location } & \multirow[b]{2}{*}{ target } & \multirow[b]{2}{*}{ Background } & \multicolumn{2}{|c|}{ Remote sensing tools } & \multirow{2}{*}{$\begin{array}{l}\text { Employed } \\
\text { Features } \\
(\mathrm{nm})\end{array}$} & \multirow[b]{2}{*}{$\begin{array}{l}\text { Processing } \\
\text { methodology }\end{array}$} & \multirow[b]{2}{*}{$\begin{array}{l}\text { Detection } \\
\text { limit (\%) }\end{array}$} & \multirow[b]{2}{*}{ Results } & \multirow[b]{2}{*}{ Reference } \\
\hline & & & $\begin{array}{l}\text { Distal } \\
\text { (GSD) }\end{array}$ & \begin{tabular}{|c|} 
Proximal \\
(Spectroscopy)
\end{tabular} & & & & & \\
\hline $\begin{array}{l}\text { Ventura, Santa } \\
\text { Barbara, USA }\end{array}$ & Natural oil seeps & $\begin{array}{c}\text { Monterey, Saugus, } \\
\text { and Fernando } \\
\text { Formations }\end{array}$ & $\begin{array}{l}\text { Probe-1 } \\
(5 \mathrm{~m})\end{array}$ & $\begin{array}{c}\text { Field } \\
\text { spectroscopy }\end{array}$ & 2300 & $\begin{array}{l}\text { Spectral } \\
\text { unmixing }\end{array}$ & 25 & Excellent & $\begin{array}{l}\text { (Ellis et al., } \\
\text { 2001) }\end{array}$ \\
\hline $\begin{array}{l}\text { Spandau, } \\
\text { Germany }\end{array}$ & $\begin{array}{l}\text { Simulated oil } \\
\text { contamination }\end{array}$ & Sand & $\begin{array}{l}\text { HyMap } \\
(4 \mathrm{~m})\end{array}$ & $\begin{array}{c}\text { In situ } \\
\text { spectroscopy }\end{array}$ & 1700 & $\begin{array}{c}\text { Color } \\
\text { composite, BR }\end{array}$ & 2.5 & Promising & $\begin{array}{c}\text { (Hörig et al., } \\
\text { 2001; Kühn et } \\
\text { al., 2004) }\end{array}$ \\
\hline $\begin{array}{l}\text { Ventura basin, } \\
\text { USA }\end{array}$ & Natural oil seeps & $\begin{array}{l}\text { Saugus and } \\
\text { Fernando } \\
\text { Formations }\end{array}$ & $\begin{array}{c}\text { HSI (not } \\
\text { specified) } \\
(3 \mathrm{~m})\end{array}$ & $\begin{array}{c}\text { Field } \\
\text { spectroscopy }\end{array}$ & 2300 & $S A M+D T$ & Unknown & Excellent & $\begin{array}{c}\text { (Prelat et al., } \\
\text { 2013) }\end{array}$ \\
\hline $\begin{array}{l}\text { Barataria Bay, } \\
\text { USA }\end{array}$ & Oil-impacted sites & coastline & $\begin{array}{c}\text { AVIRIS } \\
(3.5-7.6 \mathrm{~m})\end{array}$ & $\begin{array}{c}\text { Field } \\
\text { spectroscopy }\end{array}$ & $2300 \& 1700$ & $\begin{array}{c}\text { Feature fitting } \\
\text { via MICA }\end{array}$ & 16 & Excellent & $\begin{array}{c}\text { (Kokaly et al., } \\
\text { 2013) }\end{array}$ \\
\hline $\begin{array}{l}\text { North } \\
\text { Charleston, } \\
\text { USA }\end{array}$ & $\begin{array}{c}\text { Environmental } \\
\text { pollution (diesel, } \\
\text { crude oil, motor oil) }\end{array}$ & $\begin{array}{l}\text { Estuarine area } \\
\text { (dominated by } \\
\text { clay minerals) }\end{array}$ & $\begin{array}{l}\text { AVIRIS } \\
(20 \mathrm{~m})\end{array}$ & $\begin{array}{c}\text { Field/Lab } \\
\text { spectroscopy }\end{array}$ & $1700 \& 2300$ & $\begin{array}{c}\text { Mixture } \\
\text { simulation and } \\
\text { spectral } \\
\text { matching via } \\
\text { SAM/MF ratio }\end{array}$ & 10 & Promising & $\begin{array}{c}\text { (Smailbegovic } \\
\text { et al., 2009) }\end{array}$ \\
\hline $\begin{array}{l}\text { Doberitzer } \\
\text { Heide, } \\
\text { Germany }\end{array}$ & $\begin{array}{c}\text { Contaminations \& } \\
\text { plastics }\end{array}$ & Different soils & $\begin{array}{l}\text { HyMap } \\
(5-6 \mathrm{~m})\end{array}$ & $\begin{array}{l}\text { Lab simulation } \\
\text { \& in situ } \\
\text { spectroscopy }\end{array}$ & $\begin{array}{l}2300,1700 \\
\quad \& 1200\end{array}$ & $\mathrm{BR}$ & Unknown & Promising & $\begin{array}{c}\text { (Winkelmann, } \\
\text { 2005) }\end{array}$ \\
\hline \multirow{2}{*}{$\begin{array}{l}\text { Casper, } \\
\text { Wyoming, USA }\end{array}$} & \multirow[t]{2}{*}{ Simulated oil-show } & \multirow{2}{*}{$\begin{array}{c}\text { Sandy, clayey, and } \\
\text { dolomitic soils }\end{array}$} & $\begin{array}{c}\text { Simulated } \\
\text { WV-3 } \\
(4-7.5 \mathrm{~m})\end{array}$ & \multirow{2}{*}{$\begin{array}{c}\text { In situ } \\
\text { spectroscopy }\end{array}$} & 1700 & $\begin{array}{l}\mathrm{BR}, \mathrm{RBD}, \\
\mathrm{MF}\end{array}$ & $\sim 35$ & Promising & $\begin{array}{c}\text { Asadzadeh } \\
\text { and de Souza } \\
\text { Filho, 2016a) }\end{array}$ \\
\hline & & & \begin{tabular}{|c|}
$\begin{array}{c}\text { ProspecTIR } \\
(0.6 \mathrm{~m})\end{array}$ \\
\end{tabular} & & $2300 \& 1700$ & MTMF & 5 & Excellent & $\begin{array}{c}\text { (Scafutto, } \\
2015)\end{array}$ \\
\hline
\end{tabular}

More recently, case histories over contaminated sites in Barataria Bay marshes affected by the Deepwater Horizon spill indicated the applicability of imaging spectrometer data to assess oiled sites onshore ${ }^{1}$. Spectral feature analysis incorporated both the HC features centered at 2300 and $1700 \mathrm{~nm}$ to compare the AVIRIS (Airborne Visible/InfraRed Imaging Spectrometer) data to the reference spectra collected in the field (Table 4). The maps derived from AVIRIS datasets with different dates and spatial resolutions were demonstrated to be $\sim 90 \%$ accurate in predicting the oiled shorelines. The smallest oily target detected at $7.6 \mathrm{~m}$ Ground Sampling Distance (GSD) was shown to be $1.2 \mathrm{~m}$, which is equivalent to $16 \%$ detection limit for the sensor (Kokaly et al., 2013).

In another environmentally oriented study, a suite of clean soil samples was impregnated by $10 \%, 30 \%$, and $50 \%$ volume of four different HCs namely crude oil, motor oil, diesel, and kerosene and then measured spectrally. The compiled spectral library was then used to detect polluted sites in an estuarine area using AVIRIS data acquired at a GSD of $20 \mathrm{~m}$ (Table 4). The data successfully detected muddy and/or sandy estuarine sediments contaminated by around $10 \%$ HC contents. The HC features, however, were more apparent in the sandy samples rather than darker loamy mud (Smailbegovic et al., 2009). The study was incapable of distinguishing between the varieties of $\mathrm{HC}$ contaminations in the sediments.

1- Such case histories are noted here because of their direct relevance to oil-seep detection. 
So far, HC detection has been confined to airborne hyperspectral sensors, but some simulation experiments have demonstrated that the newly launched WorldView-3 (WV-3) satellite system can potentially be employed for direct $\mathrm{HC}$ detection using the bands coinciding with HC's $1700 \mathrm{~nm}$ feature (Table 4) (Asadzadeh and Souza Filho, 2016a). If further case studies with real datasets support this finding, the WV-3 satellite data then will be the first spaceborne instrument capable of detecting onshore oil directly and unambiguously.

\subsection{Gas-plume sensing}

Contrary to the marine environment in which gas seeps are easily noticeable, terrestrial seepage detection has been biased towards more visually evident oil seeps and, thereof, many tiny gas leakages have gone unnoticed. Generally, where the emission of such seeps occur as concentrated point sources, the escaping methane can form discernible gas-plume in the atmosphere and thus constitute detectable target. Current orbital gas remote sensing instruments like the Scanning Imaging Absorption Spectrometer for Atmospheric Chartography (SCIAMACHY) and the Greenhouse Gas Observing Satellite (GOSAT) have the capability to measure methane and other trace gasses but only in continental scales (Watanabe et al., 2015). The delineation of point source plumes at local-scale, however, is a niche that is filled with high spatial $(G S D<20 \mathrm{~m})$ and spectral resolution hyperspectral imaging instruments.

The detection and mapping of methane plumes from natural and anthropogenic sources relies on its diagnostic absorption features in the SWIR $(\sim 2.3 \mu \mathrm{m})$ and/or the LWIR $(\sim 7.6 \mu \mathrm{m})$ wavelengths. In the SWIR, AVIRIS and AVIRIS-NG are the highly explored systems for this aim. For example, high-glint AVIRIS imagery was successful in detecting the concentrated sources of $\mathrm{CH}_{4}$ in the Coal Oil Point (COP) seeps in the Santa Barbara Channel, California. In this test site, radiance data were processed using band ratioing and MODTRAN-based simulated radiance spectra to generate a 'methane index' over some known marine gas seeps (Bradley et al., 2011; Roberts et al., 2010) (Table 5). In the case of terrestrial methane mapping where emissions occur over non-uniform and heterogeneous background albedo, encouraging results were achieved by more advanced processing algorithms such as the Cluster-Tuned Matched Filter (CTMF) and the Iterative Maximum a Posteriori Differential Optical Absorption Spectroscopy (IMAP-DOAS) (Frankenberg et al., 2016; Thorpe et al., 2013) (Table 5). This approach was not only effective in detecting a plume but also was capable of quantifying the flux of $\mathrm{CH}_{4}$ in the atmosphere (see section 6-1-1).

Some parallel studies using the LWIR wavelength has already given rise to encouraging results for methane detection. For instance, data from the airborne Hyperspectral Thermal Emission Spectrometer (HyTES) at $\sim 2 \mathrm{~m}$ GSD were successful to map several individual methane plumes over oil fields in the San Joaquin Valley, California (Table 5).

Table 5. Summary of the case studies directed towards gas-plume (methane) sensing over natural seepage systems.

\begin{tabular}{|c|c|c|c|c|c|c|}
\hline Location & target & $\begin{array}{l}\text { Instrument } \\
\text { (GSD) }\end{array}$ & $\begin{array}{c}\text { Employed } \\
\text { Feature }\end{array}$ & $\begin{array}{l}\text { Processing } \\
\text { methodology }\end{array}$ & Results & Reference \\
\hline $\begin{array}{l}\text { Coal Oil Point, } \\
\text { California, USA }\end{array}$ & $\begin{array}{l}\text { Natural } \\
\text { seep } \\
\text { (offshore) }\end{array}$ & $\begin{array}{l}\text { AVIRIS } \\
(7.5 \mathrm{~m})\end{array}$ & \multirow{2}{*}{$\begin{array}{c}2.298 \mu \mathrm{m} \\
\text { (SWIR) }\end{array}$} & \begin{tabular}{|c} 
Band ratioing \\
Spectral \\
residuals
\end{tabular} & $\begin{array}{c}\text { Promisin } \\
\mathrm{g}\end{array}$ & $\begin{array}{c}\text { (Bradley et } \\
\text { al., 2011; } \\
\text { Roberts et al., } \\
\text { 2010) }\end{array}$ \\
\hline $\begin{array}{l}\text { Inglewood Oil } \\
\text { Field, Los } \\
\text { Angeles, USA }\end{array}$ & $\begin{array}{c}\text { Natural } \\
\text { seep } \\
\text { (onshore) }\end{array}$ & $\begin{array}{l}\text { AVIRIS } \\
(\sim 3 \mathrm{~m})\end{array}$ & & CTMF & $\begin{array}{c}\text { Promisin } \\
\mathrm{g}\end{array}$ & $\begin{array}{c}\text { (Thorpe et al., } \\
\text { 2013) }\end{array}$ \\
\hline
\end{tabular}




\begin{tabular}{|l|c|c|c|c|c|c|}
\hline $\begin{array}{l}\text { San Juan Basin, } \\
\text { USA }\end{array}$ & $\begin{array}{c}\text { Natural } \\
\text { seep } \\
\text { (onshore) }\end{array}$ & $\begin{array}{c}\text { AVIRIS-NG } \\
(1-3 \mathrm{~m})\end{array}$ & IMAP-DOAS & $\begin{array}{c}\text { Promisin } \\
\mathrm{g}\end{array}$ & $\begin{array}{c}\text { (Frankenberg } \\
\text { et al., 2016) }\end{array}$ \\
\hline San Joaquin & $\begin{array}{c}\text { Natural } \\
\text { Valley, }\end{array}$ & HyTES \\
California, USA & $\begin{array}{c}\text { seep? } \\
\text { (onshore) }\end{array}$ & $\begin{array}{c}7.66 \mu \mathrm{m}) \\
(\text { LWIR) }\end{array}$ & $\begin{array}{c}\text { Clutter } \\
\text { Matched } \\
\text { Filter (CMF) }\end{array}$ & $\begin{array}{c}\text { Promisin } \\
\mathrm{g}\end{array}$ & $\begin{array}{c}\text { (Hulley et al., } \\
\text { 2016) }\end{array}$ \\
\hline
\end{tabular}

Aside from the emerging airborne technology, ground-based sensing instruments have been utilized for gas seepage detection. For a comprehensive review of active ground-based systems, including laser and Lidar sensors, the reader is referred to Chapter 4 of Etiope's book (Etiope, 2015) and references therein. In the lab, reflectance spectroscopy has been further attempted to facilitate the detection of adsorbed gas (and probably aromatics/petroleum?) within clay particles. The trial with soil samples collected over three oil and gas fields indicated that meaningful spectral trends coexist over petroliferous areas (McCoy et al., 2001).

It is worth mentioning that X-band radar was indicated to be capable of detecting atmospheric seepage anomaly (ASA) above terrestrial accumulations. The scientific concept along with some case studies were discussed in a series of papers by T. Bailey (Bailey, 1996; Bailey and Grubb, 2006; Bailey and Skolnik, 1996).

\section{Microseepage remote sensing}

Since the majority of the mineralogical assemblages in microseepage systems (section 2-2) show diagnostic spectral features within VNIR-SWIR wavelengths (Hunt, 1977), they have been the focus of several remote sensing investigations. This section firstly attempts to overview the case studies available on the subject and then summarize the closely related surveys on exhumed $\mathrm{HC}$ reservoirs.

\subsection{An overview of the case-studies}

Near-surface diagenetic changes have been known for a long time (e.g. Davidson (2004) and references therein); however, the first systematic and comprehensive study on the topic was conducted only in the 1970s by Donovan (1974), which was simultaneous to the concept of 'hazy anomaly' from ERTS data. A term that was brought about to explain the peculiar subtle tonal areas observed in enhanced imagery over some productive/prospective onshore fields ${ }^{2}$ (Simpson, 1978). Thereafter, different generations of remote sensing data have been employed for this aim, each revealing further details of the diagenetic mineralogy within microseepage systems.

In this paper, we define three levels for remote sensing-derived anomalies including (i) tonal (hazy) anomaly, (ii) spectral anomaly, and (iii) mineral map (Table 6). The tonal anomaly is interpreted visually, thus its exact mineralogical attribute is not clear. The spectral anomaly is routinely derived from multispectral imagery following simple spectral analysis. In this level, altered zones are discriminated in a broad sense without attempting to discriminate mineral species (e.g. OH-bearing minerals derived from Landsat data). In latter, however, the majority of the minerals appearing in a microseepage system are either distinguished (e.g. Al-OH) or identified individually (e.g. kaolinite).

Key case studies demonstrating the application of remote sensing methods for microseepage delineation are summarized in Table 6 . We devised a variety of attributes comprising field characteristics, geologic/mineralogic features (e.g. exposed stratigraphic units), remote sensing approach (i.e. distal

2- According to our experiment, a hazy anomaly arises likely due to the dissolution/re-deposition of authigenic minerals and subsequent disappearance of the texture and fabric of the original facies. 
airborne/spaceborne and proximal sensing tools), specifications of the yielded target, ground-truthing strategy along with complementary analytical techniques, and then tabulated the case studies on their basis. In this table, we distinguished active microseepage systems with ongoing leakage to the surface from fossil (inactive) systems wherein the HC migration has halted. In addition, for clarification purposes, case studies addressing miniseeps (See section 2-1 and Fig. 1) were segregated from microseepage systems. More importantly, the potential targets revealed by fieldwork were contrasted against mapping results achieved through spectral analysis in order to emphasize the weakest link(s) on the processing chain. We made a subjective judgment about the shape of the resulting anomaly and its correspondence with the surface projection of the accumulation and the overall outcome of every case study. Subsequently, we assigned the following scores to each of the attributes: very-high>high>medium>low>slim for the former and excellent>promising>average>poor for the latter (Table 6).

Due to the significance of ground-truthing in demonstrating the capability of remote sensing, in this table, we also reported the number of collected samples, the likely spectroscopy of the sample suite, the sampling scheme (traverse vs. selective), and the locality of sampling sites 'on' and 'off' the petroliferous (altered) zones considering the same geologic unit. Ultimately, because many case studies were supplemented by geochemical and/or geophysical techniques of one type or another, we accounted for them under a category named analytical approach (Table 6). To better reflect the trends in the compiled case histories (28 individual cases), we generalized the attributes of Table 6 to construct a series of bar charts illustrated in Figs. 3-4.

Apparently, the case studies are not uniformly distributed, for the majority of them are conducted in the United States or China and paramount petroliferous regions such as the Middle East are not adequately investigated (Fig. 3a). Although the cases have evenly covered oil and gas accumulations (Fig. 3b), they are biased heavily towards the structural traps and the important stratigraphic entities are not satisfactorily incorporated (Fig. 3c). 
Table 6. Summary of the remote sensing case studies directed towards the detection and characterization of alterations induced by onshore mini-, and microseepage systems (the miniseep cases are marked in gray).

\begin{tabular}{|c|c|c|c|c|c|c|c|c|c|}
\hline \multirow[b]{2}{*}{ Location } & \multicolumn{3}{|c|}{ Field characteristics } & \multicolumn{3}{|c|}{ Geological/mineralogical features } & \multicolumn{3}{|c|}{ Remote sensing approach } \\
\hline & HC charge & Trap & $\begin{array}{l}\text { Seepage } \\
\text { activity \& } \\
\text { type }\end{array}$ & Outcrop lithology & Age & Exploration signatures & Distal tool & $\begin{array}{l}\text { Proximal } \\
\text { tool }\end{array}$ & $\begin{array}{l}\text { Processing } \\
\text { method }\end{array}$ \\
\hline \multirow{3}{*}{$\begin{array}{l}\text { Lisbon Valley, Utah, } \\
\text { USA }\end{array}$} & \multirow{3}{*}{ Oil \& gas } & \multirow{3}{*}{$\begin{array}{c}\text { Structural } \\
\text { (faulted trap) }\end{array}$} & \multirow{3}{*}{ Active } & \multirow{3}{*}{$\begin{array}{l}\text { Wingate } \\
\text { sandstone }\end{array}$} & \multirow{3}{*}{$\begin{array}{c}\text { Early } \\
\text { Jurassic }\end{array}$} & \multirow{3}{*}{$\begin{array}{l}\text { - Bleaching of red beds } \\
\text { - High clay content } \\
\text { - High precipitation of limonite and carbonate } \\
\text { - Anomalous weathering patterns }\end{array}$} & $\begin{array}{c}\text { MSS } \\
\text { TMS, TM }\end{array}$ & Spectroscopy & $\mathrm{BR}$ \\
\hline & & & & & & & $\begin{array}{l}\text { ASTER } \\
\text { JERS }\end{array}$ & Spectroscopy & ML, PCA \\
\hline & & & & & & & HyMap & $\begin{array}{c}\text { ASD } \\
\text { spectroscopy }\end{array}$ & SAM, SFF \\
\hline $\begin{array}{l}\text { Navajo, Utah, } \\
\text { USA }\end{array}$ & Oil/gas & - & Ancient & $\begin{array}{l}\text { Navajo } \\
\text { Sandstone }\end{array}$ & Jurassic & $\begin{array}{l}\text { - Iron Oxide beaching \& goethite } \\
\text { - Clays } \\
\text { - Carbonate (calcite) }\end{array}$ & HyMap & $\begin{array}{l}\text { ASD \& FTIR } \\
\text { spectroscopy }\end{array}$ & MF \\
\hline $\begin{array}{l}\text { Patrick Draw } \\
\text { Wyoming, USA }\end{array}$ & Oil & Stratigraphic & Active & $\begin{array}{l}\text { Calcareous/silty } \\
\text { sandstone }\end{array}$ & Eocene & $\begin{array}{l}\text { - Clays (illite, kaolinite/smectite) } \\
\text { - Iron oxides (ferrihydrite, goethite) }\end{array}$ & Hyperion & $\begin{array}{c}\text { ASD } \\
\text { spectroscopy }\end{array}$ & SAM \\
\hline $\begin{array}{l}\text { Santa Barbara } \\
\text { County, USA }\end{array}$ & Heavy oil & Structural & $\begin{array}{c}\text { Active } \\
\text { (Miniseep) }\end{array}$ & $\begin{array}{l}\text { Saugus and Fernando } \\
\text { Fm? }\end{array}$ & Pliocene & - Jarosite, siderite, calcite & AVIRIS & . & SAM, SFF \\
\hline $\begin{array}{l}\text { Ventura, Santa } \\
\text { Barbara, USA }\end{array}$ & Oil & Structural & $\begin{array}{c}\text { Active } \\
\text { (Miniseep) }\end{array}$ & Fernando Fm. & Pliocene & $\begin{array}{l}\text { - Halos around macroseeps characterized by } \\
\text { goethite and calcite }\end{array}$ & $\begin{array}{l}\text { Probe-1 } \\
\text { (HyMap) }\end{array}$ & - & SAM, CCSM \\
\hline $\begin{array}{l}\text { Dutton Basin, } \\
\text { Wyoming, USA }\end{array}$ & Oil & Anticline & $\begin{array}{l}\text { Ancient/ } \\
\text { inactive } \\
\text { (Miniseep) }\end{array}$ & $\begin{array}{l}\text { Nugget/Chugwater Fm. } \\
\text { (siltstone, Sandstone) }\end{array}$ & Triassic & $\begin{array}{l}\text { - Fe hydroxides (goethite) } \\
\text { - Calcite veins }\end{array}$ & AVIRIS & Spectrometer & $\mathrm{BR}$ \\
\hline $\begin{array}{l}\text { Paradfurdo, Matra } \\
\text { Mount. Hungary }\end{array}$ & Gas & $?$ & $\begin{array}{l}\text { Active? } \\
\text { (Miniseep) }\end{array}$ & $?$ & $?$ & $\begin{array}{l}\text { - Botanical anomaly } \\
\text { - Mineralogical alterations }\end{array}$ & DAIS & Spectrometer & $\begin{array}{c}\text { Unmixing } \\
\text { Knowledge-based } \\
\text { method }\end{array}$ \\
\hline $\begin{array}{l}\text { Sheep Mountain, } \\
\text { Bighorn basin, } \\
\text { Wyoming, USA }\end{array}$ & Oil & Anticline & Active & $\begin{array}{l}\text { Cleverly Fm. } \\
\text { (sandstone, shale) }\end{array}$ & Triassic & $\begin{array}{l}\text { - Absence of kaolinite in shale } \\
\text { - Red bed bleaching }\end{array}$ & TM & Spectrometer & $\mathrm{BR}$ \\
\hline \multirow{2}{*}{$\begin{array}{l}\text { North Tucano Basin, } \\
\text { Brazil }\end{array}$} & \multirow{2}{*}{ Gas } & \multirow{2}{*}{ Anticline } & \multirow{2}{*}{ Active } & \multirow{2}{*}{$\begin{array}{l}\text { Marizal Fm. \& Tona } \\
\text { Sequence } \\
\text { (Sandstone, siltstone) }\end{array}$} & \multirow{2}{*}{ Tertiary } & \multirow{2}{*}{$\begin{array}{l}\text { - Bleaching and/or limonitic yellow soils } \\
\text { - Development of clay minerals } \\
\text { - Silicification \& kaolinite depletion } \\
\text { - Geobotanical anomalies }\end{array}$} & $\begin{array}{l}\text { ASTER } \\
\text { ETM+ }\end{array}$ & $\begin{array}{c}\text { ASD } \\
\text { spectroscopy }\end{array}$ & $\begin{array}{c}\text { BR, PCA } \\
\text { SAM, MTMF, NN }\end{array}$ \\
\hline & & & & & & & TM & $\begin{array}{c}\text { ASD } \\
\text { spectroscopy }\end{array}$ & $\begin{array}{c}\text { BR } \\
(2 / 3,4 / 3)\end{array}$ \\
\hline $\begin{array}{l}\text { Remanso do Fongo, } \\
\text { Brazil }\end{array}$ & Gas & $?$ & $\begin{array}{c}\text { Active } \\
\text { (Miniseep) }\end{array}$ & Alluvial sediments & Holocene & $\begin{array}{l}\text { - Ferric iron and kaolinite } \\
\text { - Geobotanical anomalies }\end{array}$ & $\begin{array}{l}\text { ASTER } \\
\text { TM }\end{array}$ & - & $\mathrm{BR}, \mathrm{PCA}$ \\
\hline $\begin{array}{l}\text { Table Rock, } \\
\text { Wyoming, USA }\end{array}$ & Gas & Anticline & Active? & $\begin{array}{l}\text { Green River \& Wasatch } \\
\text { Fm. (sandstone, } \\
\text { mudstone, etc.) }\end{array}$ & Eocene & $\begin{array}{l}\text { - Mixed-layer illite-smectite, kaolinite, chlorite } \\
\text { - Hematite, pyrite, jarosite, gypsum } \\
\text { - Carbonate cement }\end{array}$ & TMS & $\begin{array}{c}\text { Airborne } \\
\text { spectroradiome } \\
\text { ter }\end{array}$ & $\begin{array}{l}\text { - Visual } \\
\text { interpretation } \\
\text { - BR } \\
\end{array}$ \\
\hline
\end{tabular}


Table 6 (Continue).

\begin{tabular}{|c|c|c|c|c|c|c|c|c|c|c|c|c|}
\hline \multirow{3}{*}{ Location } & \multicolumn{4}{|c|}{ Target specification } & \multicolumn{5}{|c|}{ Fieldwork and sampling } & \multirow{3}{*}{ Analytical approach } & \multirow{3}{*}{ Results } & \multirow{3}{*}{ Reference } \\
\hline & \multirow{2}{*}{ Anomaly type } & \multirow{2}{*}{ Anomaly shape } & \multirow{2}{*}{ Mapped target(s) } & \multirow{2}{*}{\begin{tabular}{|c|} 
Correspon \\
dence
\end{tabular}} & \multicolumn{2}{|c|}{ Locality } & \multicolumn{2}{|c|}{ Strategy } & \multirow{2}{*}{ No \# } & & & \\
\hline & & & & & On & Off & Selective & Traverse & & & & \\
\hline \multirow{3}{*}{$\begin{array}{l}\text { Lisbon Valley, } \\
\text { USA }\end{array}$} & Spectral anomaly & \multirow{3}{*}{$\begin{array}{l}\text { Elongated patches at } \\
\text { the hinge points of } \\
\text { the fold }\end{array}$} & $\begin{array}{l}\text { Rocks poor in limonite } \\
\text { with high clays }\end{array}$ & Medium & $x$ & $\mathrm{x}$ & $\mathrm{x}$ & 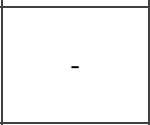 & 15 & \begin{tabular}{|l|} 
XRD \\
- Petrography \\
- Whole-rock geochemistry
\end{tabular} & Promising & $\begin{array}{c}\text { (Segal and Merin, } \\
\text { 1989; Segal et al., } \\
1986)\end{array}$ \\
\hline & Mineral map & & Bleached facies & Medium & $x$ & - & $\mathrm{x}$ & - & 7 & \begin{tabular}{|l|} 
- ICP-AES \\
- Petrography
\end{tabular} & Promising & $\begin{array}{c}\text { (Petrovic et al., } \\
\text { 2008) }\end{array}$ \\
\hline & Mineral map & & $\begin{array}{l}\text { Kaolinite, calcite, } \\
\text { hematite }\end{array}$ & Medium & $x$ & $\mathrm{x}$ & $\mathrm{x}$ & - & 37 & \begin{tabular}{|l} 
- Petrography \\
- ICP-MS/AES \\
- Isotope Study: O, C
\end{tabular} & Promising & $\begin{array}{l}\text { (Petrovic et al., } \\
\text { 2012) }\end{array}$ \\
\hline Navajo; USA & Mineral map & $\begin{array}{c}\text { Elongated patches of } \\
\text { minerals }\end{array}$ & $\begin{array}{c}\text { Goethite, hematite, } \\
\text { carbonate }\end{array}$ & Medium & $x$ & $x$ & $x$ & - & 170 & $\begin{array}{l}\text { - Petrography } \\
\text { - XRD \& ICP-MS } \\
\end{array}$ & Excellent & $\begin{array}{c}\text { (Bowen et al., } \\
\text { 2007) }\end{array}$ \\
\hline $\begin{array}{l}\text { Patrick Draw; } \\
\text { USA }\end{array}$ & Mineral map & Scattered patches & Bleaching? & Low & $x$ & - & $x$ & - & 40 & \begin{tabular}{|l|} 
- ICP \\
- XRD \\
- Isotope Study: C \\
\end{tabular} & Average & $\begin{array}{c}\text { (Khan and } \\
\text { Jacobson, 2008) }\end{array}$ \\
\hline $\begin{array}{l}\text { Santa Barbara; } \\
\text { USA }\end{array}$ & Mineral map & Scattered patches & $?$ & Not verified & - & - & - & - & - & - & Poor & (Freeman, 2003) \\
\hline Ventura; USA & Mineral map & Small patches & Goethite & Slim & - & - & - & - & - & - & Average & $\begin{array}{l}\text { (van Der Meer et } \\
\text { al., 2002) }\end{array}$ \\
\hline $\begin{array}{l}\text { Dutton Basin, } \\
\text { Wyoming, } \\
\text { USA }\end{array}$ & Mineral map & Pointy & Goethite & Medium & $x$ & - & $x$ & - & 3 & $-X R D$ & Average & $\begin{array}{c}\text { (Jengo and } \\
\text { Vincent, 1999) }\end{array}$ \\
\hline $\begin{array}{l}\text { Paradfurdo, } \\
\text { Hungary }\end{array}$ & Spectral anomaly & Scattered patches & Clays? & Medium & $x$ & - & $x$ & - & 56 & - & Promising & $\begin{array}{l}\text { (van der Werff, } \\
\text { 2006) }\end{array}$ \\
\hline $\begin{array}{l}\text { Sheep Mount. } \\
\text { USA }\end{array}$ & Tonal anomaly & $?$ & Bleaching & $?$ & $x$ & $x$ & $x$ & - & Several & $\begin{array}{l}\text { - XRD } \\
\text { - Ph measurement }\end{array}$ & Average & $\begin{array}{c}\text { (Malhotra et al., } \\
\text { 1989) }\end{array}$ \\
\hline & Spectral anomaly & $\begin{array}{l}\text { Scattered over the } \\
\text { field }\end{array}$ & Clays, iron oxides & Medium & $\mathrm{x}$ & - & $x$ & - & 7 & - Gasometry & Promising & $\begin{array}{c}\text { (Lammoglia et al., } \\
\text { 2008) }\end{array}$ \\
\hline $\begin{array}{l}\text { North Iucano } \\
\text { Basin, Brazil }\end{array}$ & Spectral anomaly & $\begin{array}{l}\text { Integrated over } \\
\text { gaseous anomaly }\end{array}$ & Iron oxides & High & $\mathrm{x}$ & - & - & $\mathrm{x}$ & 25 & $\begin{array}{l}\text { - Gasometry } \\
\text { - Radiometry } \\
\text { - Petrography }\end{array}$ & Promising & $\begin{array}{l}\text { (Almeida-Filho et } \\
\text { al., 2002; Almeida- } \\
\text { Filho et al., 1999) }\end{array}$ \\
\hline $\begin{array}{l}\text { Remanso do } \\
\text { Fongo, Brazil }\end{array}$ & Spectral anomaly & Integrated & $\begin{array}{l}\text { Kaolinite } \\
\text { iron oxides }\end{array}$ & Medium & - & - & - & - & - & $\begin{array}{l}\text { - Radiometry } \\
\text { - Magnetic survey }\end{array}$ & Average & $\begin{array}{c}\text { (Curto et al., 2011; } \\
\text { Souza Filho et al., } \\
\text { 2008) }\end{array}$ \\
\hline $\begin{array}{l}\text { Table Rock, } \\
\text { USA }\end{array}$ & $\begin{array}{l}\text { Tonal Anomaly \& } \\
\text { Mineral map }\end{array}$ & $\begin{array}{l}\text { Integrated along the } \\
\text { crest of anticline }\end{array}$ & $?$ & High & $x$ & - & - & $x$ & $10 \mathrm{~s}$ & $\begin{array}{l}\text { - XRD } \\
\text { - Petrography } \\
\text { - Ph measurement }\end{array}$ & Average & $\begin{array}{l}\text { (Marrs and } \\
\text { Paylor., 1987) }\end{array}$ \\
\hline
\end{tabular}


Table 6 (Continue).

\begin{tabular}{|c|c|c|c|c|c|c|c|c|c|}
\hline \multirow[b]{2}{*}{ Location } & \multicolumn{3}{|c|}{ Field characteristics } & \multicolumn{3}{|c|}{ Geological/mineralogical features } & \multicolumn{3}{|c|}{ Remote sensing approach } \\
\hline & HC charge & Trap & $\begin{array}{c}\text { Seepage } \\
\text { activity \& } \\
\text { type }\end{array}$ & Outcrop lithology & Age & Exploration signatures & Distal tool & $\begin{array}{l}\text { Proximal } \\
\text { tool }\end{array}$ & $\begin{array}{l}\text { Processing } \\
\text { method }\end{array}$ \\
\hline \multirow{2}{*}{$\begin{array}{l}\text { Palm Valley, } \\
\text { Amadeus basin, } \\
\text { Australia }\end{array}$} & \multirow{2}{*}{ Gas } & \multirow{2}{*}{ Anticline } & \multirow{2}{*}{ Active } & \multirow{2}{*}{$\begin{array}{l}\text { Hermannsburg } \\
\text { sandstone }\end{array}$} & \multirow{2}{*}{ Devonian } & \multirow{2}{*}{$\begin{array}{l}\text { - Fe anomaly (magnetite high and hematite low) } \\
\text { - Calcrete (dolomite, calcite) } \\
\text { - Clays (montmorillonite and kaolinite) } \\
\text { - Surface weathering/crust }\end{array}$} & $\begin{array}{l}\text { TM } \\
\text { TMS }\end{array}$ & $\begin{array}{c}\text { IRIS } \\
\text { spectrometer }\end{array}$ & $\mathrm{BR}$ \\
\hline & & & & & & & $\begin{array}{l}\text { GEOSCAN } \\
\text { AMSS }\end{array}$ & $\begin{array}{c}\text { IRIS } \\
\text { spectrometer }\end{array}$ & SFF \\
\hline $\begin{array}{l}\text { Los Chihuidos High, } \\
\text { Argentina }\end{array}$ & Gas \& oil & \multicolumn{2}{|c|}{ Exhumed reservoir } & $\begin{array}{l}\text { Huincul Fm. } \\
\text { (sandstone) }\end{array}$ & $\begin{array}{c}\text { Upper } \\
\text { Cretaceous }\end{array}$ & - Bleaching of redbeds attributed to $\mathrm{HC}$ migrations & ETM+ & 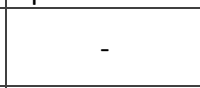 & $\begin{array}{c}\text { Visual } \\
\text { Interpretation }\end{array}$ \\
\hline $\begin{array}{l}\text { Baku Region, } \\
\text { Azerbaijan }\end{array}$ & Oil & Structural & $\begin{array}{c}\text { Active } \\
\text { (miniseep) }\end{array}$ & $\begin{array}{l}\text { Alluvial (Clay and } \\
\text { sand) }\end{array}$ & Pliocene & $\begin{array}{l}\text { - Clays (Montmorillonite, chlorite, illite, kaolinite) } \\
\text { - Bleached/discolored red sandstones } \\
\text { - Ferrous carbonate }\end{array}$ & $\begin{array}{l}\text { ASTER } \\
\text { InSAR }\end{array}$ & $\begin{array}{c}\text { FieldSpec FR } \\
\text { spectrometer }\end{array}$ & VMESMA \\
\hline Ramhormoz, Iran & Oil \& gas & Anticline & $\begin{array}{c}\text { Active } \\
\text { (miniseep) }\end{array}$ & $\begin{array}{l}\text { Gachsaran \& Mishan } \\
\text { Fm. (Marl) }\end{array}$ & Miocene & $\begin{array}{l}\text { - The formation of gypsum and S in marly } \\
\text { limestone. } \\
\text { - Removal of Fe oxides and calcite }\end{array}$ & $\begin{array}{c}\text { ASTER } \\
\text { WorldView-2 }\end{array}$ & \begin{tabular}{|l} 
ASD \\
spectroscopy \\
(In the lab)
\end{tabular} & $\begin{array}{l}\text { BR, PCA } \\
\text { BRT }\end{array}$ \\
\hline $\begin{array}{l}\text { Masjed Soleiman, } \\
\text { Iran }\end{array}$ & Oil & Anticline & $\begin{array}{c}\text { Active } \\
\text { (miniseep) }\end{array}$ & $\begin{array}{l}\text { Gachsaran Fm. } \\
\text { (gypsum) }\end{array}$ & Miocene & $\begin{array}{l}\text { - Decrease in gypsum/anhydrite (Gach-e-Turush) } \\
\text { - Increase in calcite, dolomite, aragonite, Sulfur (S) } \\
\text { - Jarosite, Na-alunite, illite, montmorillonite }\end{array}$ & - & - & - \\
\hline $\begin{array}{l}\text { Kor Mor Field, } \\
\text { Kurdistan, Iraq }\end{array}$ & Oil & Anticline & Active & $\begin{array}{l}\text { Upper \& lower Fars } \\
\text { Fm. (sandstone, } \\
\text { siltstone, marl) }\end{array}$ & Miocene & $\begin{array}{l}\text { - Calcite, dolomite } \\
\text { - Illite, chlorite } \\
\text { - Iron oxides }+ \text { jarosite }\end{array}$ & $\begin{array}{l}\text { ETM+ } \\
\text { ASTER }\end{array}$ & $\begin{array}{l}\text { ASD } \\
\text { spectroscopy } \\
\text { (In the lab) } \\
\end{array}$ & $\begin{array}{l}\text { BR, PCA } \\
\text { SFF, SAM }\end{array}$ \\
\hline $\begin{array}{l}\text { Qiulitage Anticline, } \\
\text { China }\end{array}$ & Oil & Thrust-and-fold & Active & $\begin{array}{l}\text { Red beds (sandstone } \\
\text { \& gypsum) }\end{array}$ & Pliocene & $\begin{array}{l}\text { - Bleaching } \\
\text { - Carbonates \& gypsum } \\
\text { - Clay \& OH-bearing minerals }\end{array}$ & ASTER & $\begin{array}{l}\text { ASD } \\
\text { spectroscopy } \\
\text { (In the lab) }\end{array}$ & $\begin{array}{c}\text { BR } \\
(2 / 1,4 / 9)\end{array}$ \\
\hline $\begin{array}{l}\text { Fula'erji, Songliao } \\
\text { Basin, China }\end{array}$ & Heavy oil & Stratigraphic & Inactive & Alluvial flood-plain & Holocene & - Bleaching and clay anomaly? & ETM+ & $x_{1}$ & BR, PCA \\
\hline $\begin{array}{l}\text { Dushanzi Anticline, } \\
\text { China }\end{array}$ & Oil & Structural & Active & $\begin{array}{l}\text { Red beds interbedded } \\
\text { with conglomerate }\end{array}$ & Pliocene & $\begin{array}{l}\text { - Bleaching (hem- }-\mathrm{Fe}^{3+} \text { ) to (para- }-\mathrm{Fe}^{3+} \text { ) } \\
\text { - Carbonates (calcite, siderite) } \\
\text { - Chlorite }\end{array}$ & ASTER & $\begin{array}{c}\text { ASD } \\
\text { spectroscopy } \\
\text { (In the lab) }\end{array}$ & $\begin{array}{c}\text { BR } \\
(2 / 1,4 / 6,4 / 8)\end{array}$ \\
\hline $\begin{array}{l}\text { Karamay Field, } \\
\text { China }\end{array}$ & Oil \& gas & Structural & Active & $\begin{array}{l}\text { Sandy breccia, } \\
\text { coarse sandstone }\end{array}$ & Triassic & $\begin{array}{l}\text { - Clays (Chlorite, montmorillonite) } \\
\text { - Carbonate (siderite) }\end{array}$ & Hyperion & $\begin{array}{c}\text { ASD } \\
\text { spectroscopy }\end{array}$ & MTMF, SAM \\
\hline $\begin{array}{l}\text { Lake Albert Basin, } \\
\text { Uganda }\end{array}$ & Oil \& gas & Structural & Active & Alluvial Sediments & $\begin{array}{l}\text { Pleistocene- } \\
\text { Holocene }\end{array}$ & $?$ & $\begin{array}{l}\text { ASTER/ETM } \\
\text { QuickBird }\end{array}$ & - & $?$ \\
\hline Ordos Basin, China & Oil & Structural? & Active & $\begin{array}{l}\text { Yanchang Fm. } \\
\text { (Sandstone, siltstone) }\end{array}$ & \begin{tabular}{|c|} 
Upper \\
Triassic \\
\end{tabular} & $\begin{array}{l}\text { - Clays (Illite, kaolinite) } \\
\text { - Carbonates (Siderite, calcite) }\end{array}$ & CASI/SASI & \begin{tabular}{|c|} 
ASD \\
spectroscopy
\end{tabular} & SFF \\
\hline $\begin{array}{l}\text { East-Sichuan Fold } \\
\text { Belt, China }\end{array}$ & gas & Structural & Active & $?$ & Triassic? & $\begin{array}{l}\text { - Clays (montmorillonite, illite, kaolinite, chlorite) } \\
\text { - Carbonates (calcite and dolomite) } \\
\text { - Ferric \& ferrous iron }\end{array}$ & TM & $\begin{array}{c}\text { Hitachi } \\
\text { VIS-NIR } \\
\text { spectrometer }\end{array}$ & $\mathrm{BR}$ \\
\hline
\end{tabular}


Table 6 (Continue).

\begin{tabular}{|c|c|c|c|c|c|c|c|c|c|c|c|c|}
\hline \multirow{3}{*}{ Location } & \multicolumn{4}{|c|}{ Target specification } & \multicolumn{5}{|c|}{ Fieldwork and sampling } & \multirow{3}{*}{$\begin{array}{l}\text { Analytical } \\
\text { approach }\end{array}$} & \multirow{3}{*}{ Results } & \multirow{3}{*}{ Reference } \\
\hline & \multirow{2}{*}{ Anomaly type } & \multirow{2}{*}{ Anomaly shape } & \multirow{2}{*}{ Mapped target(s) } & \multirow{2}{*}{$\begin{array}{c}\text { Correspon } \\
\text { dence }\end{array}$} & \multicolumn{2}{|c|}{ Locality } & \multicolumn{2}{|c|}{ Strategy } & \multirow{2}{*}{ No \# } & & & \\
\hline & & & & & On & Off & Selective & Traverse & & & & \\
\hline \multirow[t]{2}{*}{$\begin{array}{l}\text { Palm Valley, } \\
\text { Australia }\end{array}$} & Tonal anomaly & Integrated & Probably iron oxides & Medium & $\mathrm{x}$ & $?$ & 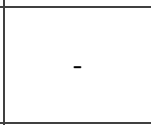 & $\mathrm{x}$ & $\begin{array}{c}60 \\
\text { spectra }\end{array}$ & $\begin{array}{l}\text { - Gasometry } \\
\text { - Isotope studies } \\
\text { - Ph measurement }\end{array}$ & Average & $\begin{array}{l}\text { (Simpson et al., } \\
\text { 1991) }\end{array}$ \\
\hline & Mineral Map & Integrated & Calcite, silica & Medium & $x$ & - & $x$ & - & several & - & Average & (Agar, 1999) \\
\hline $\begin{array}{l}\text { Los Chihuidos } \\
\text { High, } \\
\text { Argentina } \\
\end{array}$ & spectral anomaly & Pervasive halo & Bleached beds & High & $x$ & $x$ & - & $\left|\begin{array}{c}x \\
\text { (7 profiles) }\end{array}\right|$ & 40 & $\begin{array}{l}\text { - Petrography } \\
\text { - ICP-AES/MS; XRD } \\
\text { - FTIR spectroscopy }\end{array}$ & Promising & $\begin{array}{l}\text { (Rainoldi et al., } \\
\text { 2014) }\end{array}$ \\
\hline $\begin{array}{l}\text { Baku Region, } \\
\text { Azerbaijan }\end{array}$ & Mineral Map & $\begin{array}{c}\text { Patchy above mud } \\
\text { volcanos }\end{array}$ & $\begin{array}{l}\text { Smectite, } \\
\text { kaolinite }\end{array}$ & - & $x$ & - & $\mathrm{x}$ & - & several & 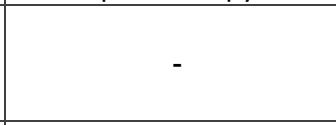 & Promising & $\begin{array}{c}\text { (Scholte, 2005; } \\
\text { Scholte et al., } \\
\text { 2003) } \\
\end{array}$ \\
\hline $\begin{array}{l}\text { Ramhormoz, } \\
\text { Iran }\end{array}$ & Spectral anomaly & Pointy & Bleaching? & Slim & $x$ & - & $x$ & - & 88 & $\begin{array}{l}\text { - ICP-OES; XRD } \\
\text { - Stable isotopes: C,O,S }\end{array}$ & Poor & $\begin{array}{l}\text { (Salati et al., } \\
\text { 2014a; Salati et } \\
\text { al., 2014b) }\end{array}$ \\
\hline \begin{tabular}{|l} 
Masjed \\
Soleiman, \\
Iran \\
\end{tabular} & - & - & - & - & $x$ & - & $x$ & - & 18 & $\begin{array}{l}\text { - ICP-AES; XRD } \\
\text { - Isotope Study: C } \\
\text { - Ph measurement }\end{array}$ & Promising & $\begin{array}{c}\text { (Tangestani and } \\
\text { Validabadi, 2014) }\end{array}$ \\
\hline $\begin{array}{l}\text { Kor Mor } \\
\text { Field; Iraq }\end{array}$ & Mineral Map & Pervasive apical & Bleaching, calcite & Very high & $\mathrm{x}$ & - & $\mathrm{x}$ & - & $>30$ & ( & Promising & $\begin{array}{c}\text { (Perry and Kruse, } \\
2010)\end{array}$ \\
\hline $\begin{array}{l}\text { Qiulitage Ant. } \\
\text { China }\end{array}$ & Spectral anomaly & Pervasive apical & Bleached beds & High & $x$ & $x$ & $x$ & - & 10 & $\begin{array}{l}\text { - Petrography } \\
\text { - SEM; XRD }\end{array}$ & Excellent & (Shi et al., 2012) \\
\hline $\begin{array}{l}\text { Fula'erji, } \\
\text { China }\end{array}$ & Tonal anomaly & $?$ & $?$ & $?$ & - & - & - & - & 19 & $\begin{array}{l}\text { - Magnetic susceptibility } \\
\text { - Delta carbonate }\end{array}$ & Poor & $\begin{array}{c}\text { (Zhang et al., } \\
\text { 2009) }\end{array}$ \\
\hline $\begin{array}{l}\text { Dushanzi Ant. } \\
\text { China }\end{array}$ & Tonal anomaly & Pervasive apical & $?$ & Very high & $x$ & - & $x$ & - & 8 & $\begin{array}{l}\text { - XRD; XRF } \\
\text { - Mössbaur spectroscopy } \\
\text { - XANES }\end{array}$ & Excellent & $\begin{array}{l}\text { (Fu et al., 2007; } \\
\text { Zheng et al., 2010) }\end{array}$ \\
\hline $\begin{array}{l}\text { Karamay } \\
\text { Field, China }\end{array}$ & Mineral Map & Narrow linear patches & Siderite, clays & Slim & $x$ & - & - & $x ?$ & $?$ & - & Poor & (Qin et al., 2016) \\
\hline $\begin{array}{l}\text { Lake Albert, } \\
\text { Uganda }\end{array}$ & $\begin{array}{l}\text { Microseepage } \\
\text { anomaly? }\end{array}$ & Pervasive apical & Altered units? & Very high & - & - & - & - & - & - & Excellent & $\begin{array}{c}\text { (Frassy et al., } \\
2015)\end{array}$ \\
\hline \begin{tabular}{|l|} 
Ordos Basin, \\
China
\end{tabular} & Mineral map & $\begin{array}{l}\text { Scattered along the } \\
\text { field }\end{array}$ & $\begin{array}{l}\text { Siderite, calcite, illite, } \\
\text { kaolinite }\end{array}$ & Medium & $\mathrm{x}$ & - & $x$ & - & 12 & \begin{tabular}{|l|} 
XRD \\
- AAS \\
\end{tabular} & Average & (Chen et al., 2016) \\
\hline $\begin{array}{l}\text { East-Sichuan, } \\
\text { China }\end{array}$ & Spectral anomaly & Scattered \& patchy & Clays, bleaching facies & Low & $x$ & $x$ & - & $x$ & 23 & $\begin{array}{l}\text { - XRD } \\
\text { - Chemical analysis }\end{array}$ & Poor & $\begin{array}{l}\text { (Wang and Ding, } \\
\text { 2000) }\end{array}$ \\
\hline
\end{tabular}

The acronyms used in the table are the following: TM: Landsat Thematic Mapper, ETM+: Landsat Enhanced Thematic Mapper plus, TMS: Thematic Mapper Simulator, ASTER: The Advanced Spaceborne Thermal Emission and Reflection Radiometer, ASD: Analytical Spectral Devices, BR: Band Ratioing, PCA: Principal Component Analysis, ML: Maximum Likelihood, SAM: Spectral Angle Mapper, SFF: Spectral Feature Fitting, MF: Matched Filtering, MTMF: Mixture Tuned Matched Filtering, cCSM: Cross-Correlogram Spectral Match, NN: Neural Network, VMESMA: Variable Multiple Endmember Spectral Mixture Analysis, BRT: Boosted Regression Tree, XANES: X-ray Absorption Near-edge Structure analysis, XRD: X-Ray Diffraction, SEM: Scanning Electron Microscope, XRF: X-Ray Fluorescence, ICP: Inductively Coupled Plasma, AAS: Atomic Absorptio

Spectroscopy. 


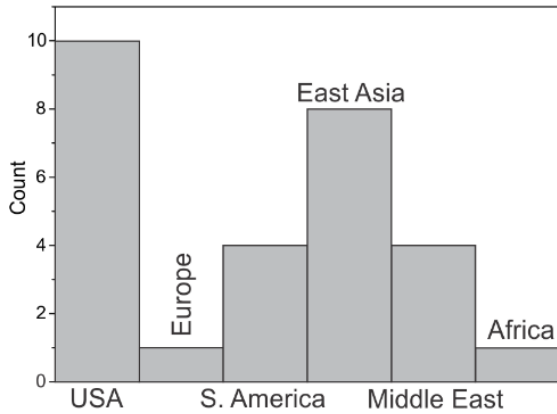

a) The Case Studies by Location

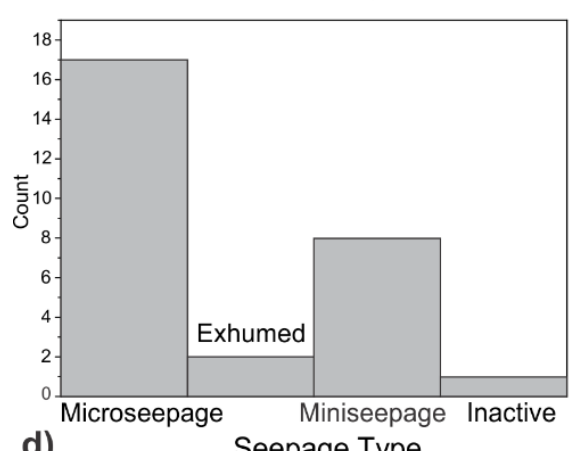

d)

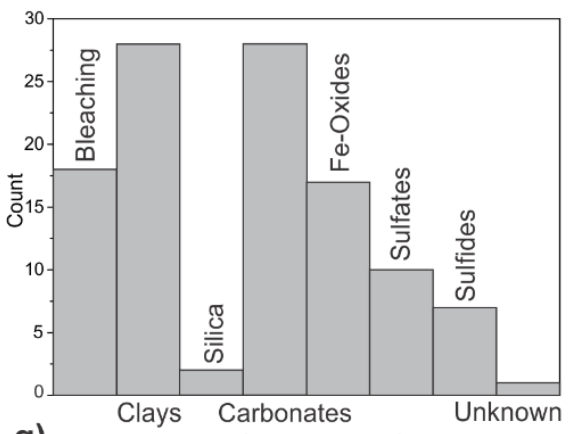

g) Exploration (Mineralogic) Targets

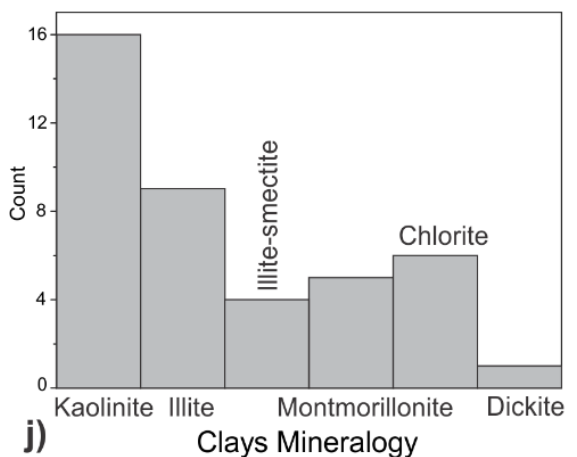

j) Clays Mineralogy

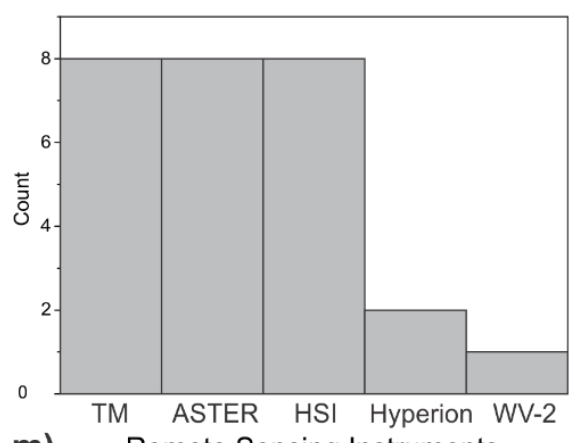

m) Remote Sensing Instruments

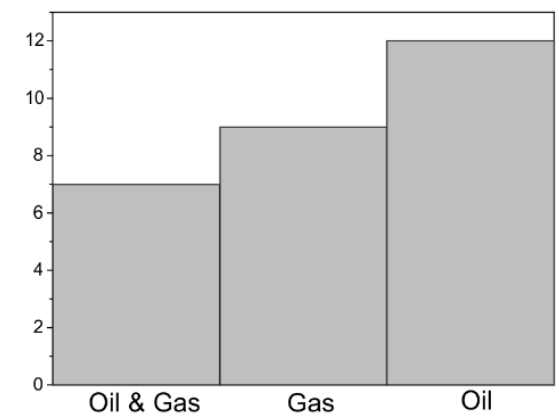

b)

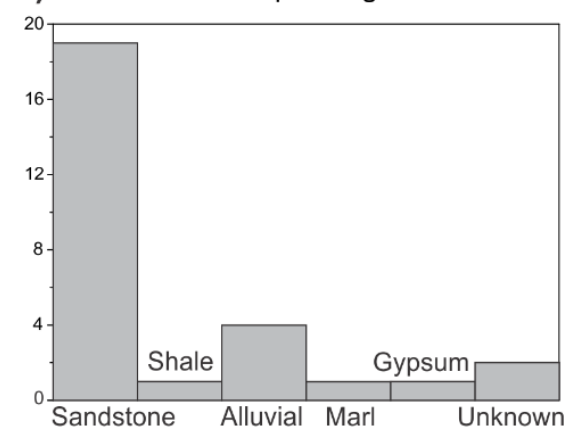

e) Outcrop Lithology (Host Rock)

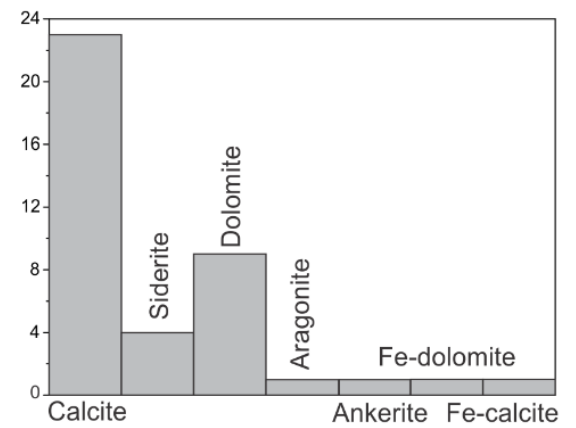

h)

Carbonates Mineralogy

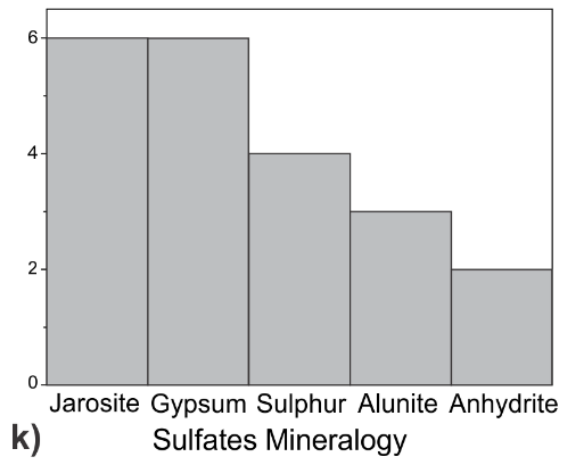

k) Sulfates Mineralogy

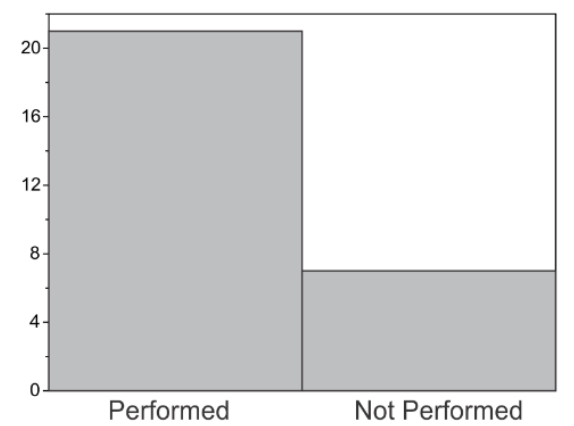

n)

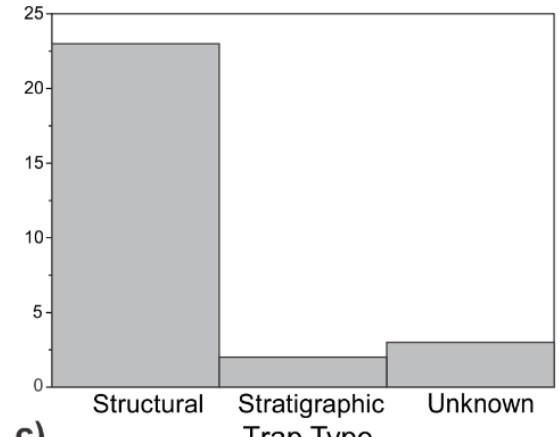

c)

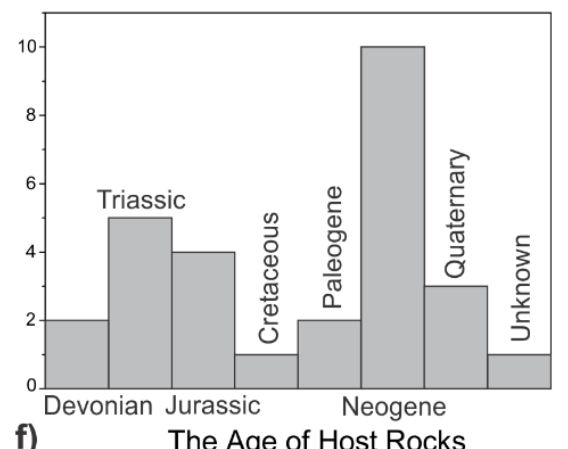

f) The Age of Host Rocks

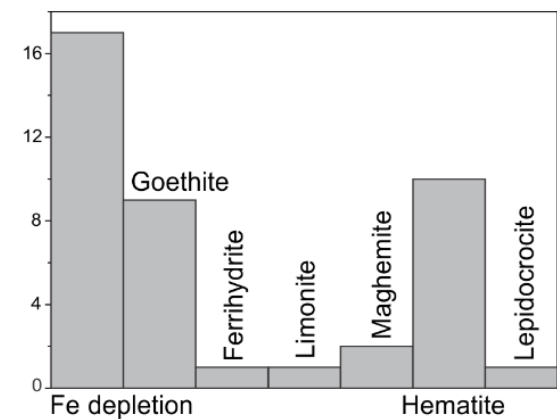

I) Iron Oxides Mineralogy

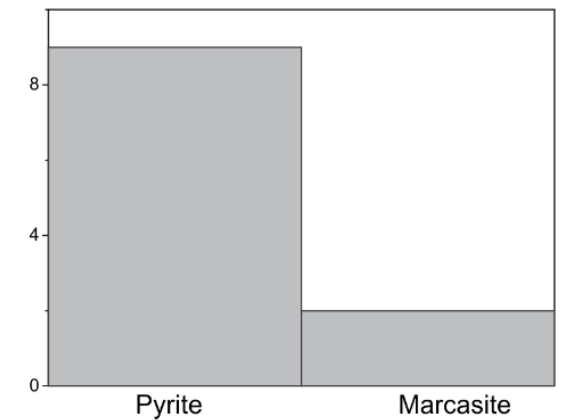

L) Sulfides Mineralogy

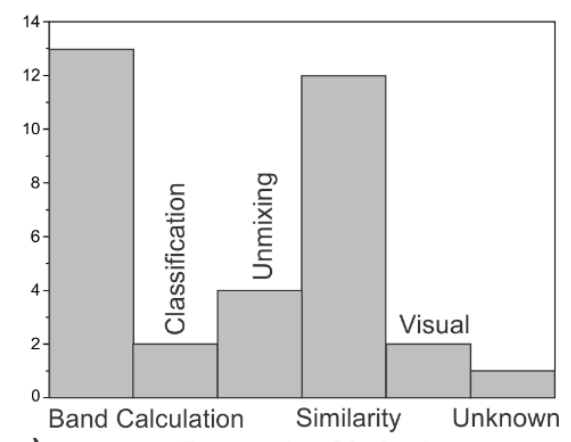

o) Processing Methods 
Fig 3. Bar chart representation of different attributes of the case studies summarized in Table 6. a) distribution of case studies around the world. b) charge of the reservoir/accumulation c) type of trap. d) seepage type (microseep vs miniseep) and activity. e) exposed lithologic units affected by microseepage system (here sandstone represents sandstone, siltstone, and conglomerate). f) generalized age of the host rocks (Quaternary encompasses Holocene and Pliocene sediments). g) described mineralogical signature of the alteration array (here the bleaching is distinguished from the excess of iron oxides). $\mathbf{h}$ ) reported mineralogy of carbonates. i) detailed mineralogy of iron oxides. j) diversity of clay species. $\mathbf{k} \&$ I) mineralogy of sulfates and sulfides. $\mathbf{m}$ ) deployed remote sensing instruments (all the Landsat series are reported as TM and airborne hyperspectral imaging systems as $\mathrm{HSI}$ ). $n$ ) number of cases performing field/lab spectroscopy. o) processing methods employed in the analysis chain (band calculation indicate PCA and band ratio techniques). For more explanation about the processing methods, the reader is referred to (Asadzadeh and Souza Filho, 2016b). Fig.3g-3k were prepared by incorporating the data from Table 7.

In terms of seepage type, several case studies have focused on miniseeps (Fig. 3d), which according to the definition provided in section 2-1, constitute smaller point targets typically off the accumulations. The chiefly accounted host rocks over microseepage systems are sandstones and other relevant clastic sediments (Fig. 3e) that extend in time from Devonian to Holocene (Fig. 3f). This bias is likely because the footprints left by migrating $\mathrm{HC}$ in these units are vast and easily recognizable. Concerning the type of alteration mineralogy, iron oxides (i.e. bleaching and/or enrichment) sounds to be the dominant entity followed closely by clays and carbonates (Fig. 3g). Within each of these assemblages, a variety of minerals were observed, albeit some individuals came up to be more important. For example, while calcite is the dominant recorded carbonate (Fig. 3h) dolomite and siderite can be potentially important targets. In the literature, great emphasis has been given to kaolinite as the most prevalent clay alteration; however, our graph (Fig. 3j) clearly shows that other clay minerals such as illite, smectites, and chlorites are equally significant and indicative of diagenetic facies. Likewise, iron oxides and sulfates (Fig. $3 \mathrm{i}$ and $3 \mathrm{k}$ ) assemblages reveal remarkably rich varieties of spectrally active minerals.

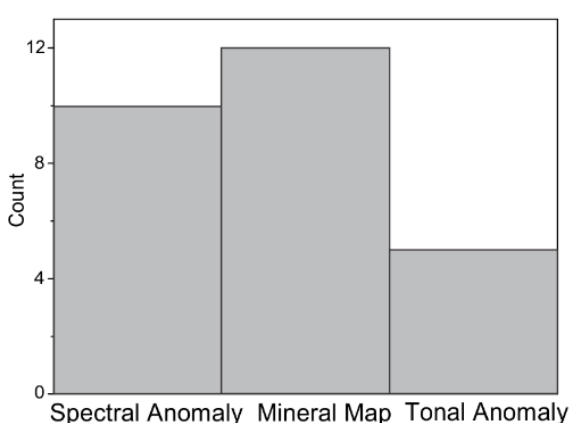

a)

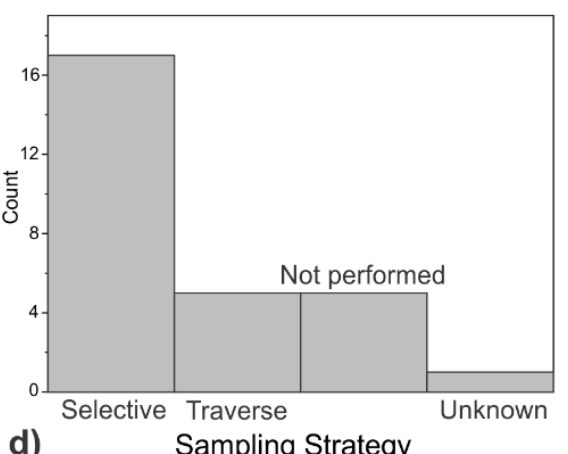

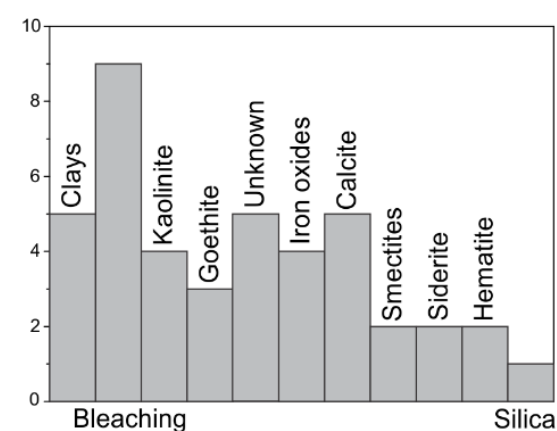

b)

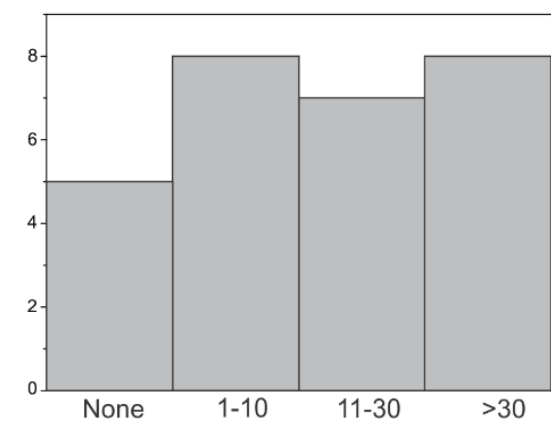

e)

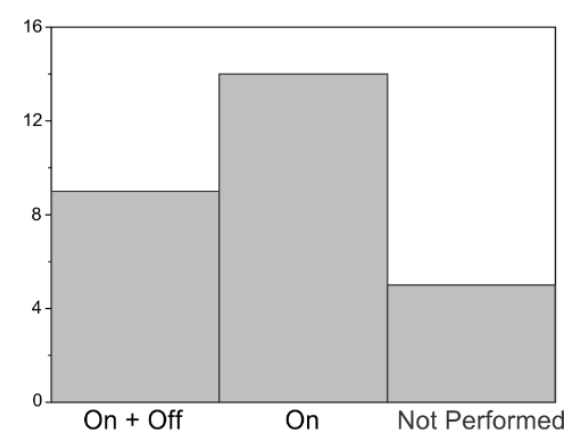

c)

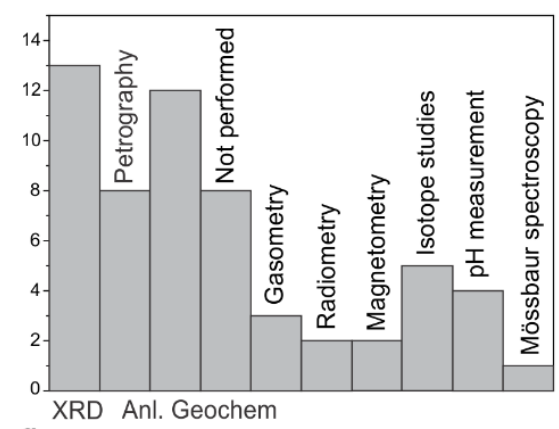

f) Supplementary Exploration Methods

Fig 4. Bar chart representation of different attributes of the case studies summarized in Table 6. a) different types of anomalies yielded from remote sensing imageries. b) mapped targeted minerals. c) locality of the samples collected in outcrop studies defined to be 'on' or 'off' the affected zones. d) strategy in sample collection. e) number of samples collected in each case study. f) supplementary exploration methods. The analytical geochemistry (Anl. Geochem) column depicts XRF, ICP-MS/AES, and other analytical methods commonly used to analyze major/minor/trace elements. 
In the outlined case studies, different generations of remote sensing instrument have been employed (Fig. $3 \mathrm{~m}$ ) to map the anomalous zones and field/lab spectroscopy has been regularly used to verify the results (Fig. 3n). Because the processing of spectral data has relied largely on simple techniques such as band ratios (Fig. 3o), many of the yielded anomalies reside in the category of spectral anomaly (Fig. 4a). As a result, the variation/pattern in the mineralogy of microseepage systems is ill-understood (Fig. 4b). According to the adopted sampling strategies, a large proportion of the cases have collected <30 samples from localities 'on' the affected zones in a selective manner (Figs. 4c-4e). Ultimately, the studies have been supplemented by a variety of analytical techniques, including petrography and XRD analysis (Fig. $4 \mathrm{f}$ ).

\subsection{Exhumed HC reservoirs}

There is a constellation of well-explored case studies on exhumed HC reservoirs/fairways in southwestern US basins (Table 7) within which the documented mineralogical suite (in microscopic, outcrop, and occasionally regional scales) remarkably resemble the microseepage-induced alterations discussed earlier (Eichhubl et al., 2004; Garden et al., 2001; Rainoldi et al., 2014). We speculate that such cases, which are characterized by intense alteration and chemical bleaching (e.g. Beitler (2005)), exemplify an endmember of the spectrum of alterations induced by HC migration/entrapment. Therefore, a deeper understanding of such changes could be an asset to the understanding of microseepage phenomenon and the characters it retains in different geologic settings.

Table 7. Summary of case studies directed towards exhumed $\mathrm{HC}$ reservoirs and paleo-fluid fairways in permeable sedimentary facies. The summarized mineralogical contents of this table were incorporated into bar charts of Fig. 3.

\begin{tabular}{|c|c|c|c|c|c|c|}
\hline Location & Target & $\begin{array}{l}\text { Outcrop } \\
\text { Lithology }\end{array}$ & Alteration signatures & $\begin{array}{l}\text { Outcrop } \\
\text { study }\end{array}$ & $\begin{array}{l}\text { Mapping } \\
\text { tool(s) }\end{array}$ & $\begin{array}{c}\text { Referenc } \\
\text { e }\end{array}$ \\
\hline $\begin{array}{l}\text { Valley of Fire; } \\
\text { NV, USA }\end{array}$ & $\begin{array}{l}\text { Exhumed } \\
\text { reservoir }\end{array}$ & $\begin{array}{c}\text { Aztec } \\
\text { sandstone }\end{array}$ & $\begin{array}{l}\text { - Clay minerals (kaolinite, illite/smectite, dickite) } \\
\text { - Goethite \& hematite banding (without pyrite) } \\
\text { - Quartz overgrowth \& carbonate cement } \\
\text { - Sulfates (alunite \& jarosite) }\end{array}$ & $x$ & $\begin{array}{l}\text { Color aerial } \\
\text { photograph }\end{array}$ & $\begin{array}{l}\text { (Eichhubl } \\
\text { et al., } \\
2004)\end{array}$ \\
\hline $\begin{array}{l}\text { Moab } \\
\text { anticline, } \\
\text { Utah, USA }\end{array}$ & $\begin{array}{l}\text { Exhumed } \\
\text { reservoir }\end{array}$ & $\begin{array}{c}\text { Entrada } \\
\text { sandstone }\end{array}$ & $\begin{array}{l}\text { - Iron oxides } \\
\text { - Calcite cementation } \\
\text { - Pyrite, ankerite, kaolinite } \\
\end{array}$ & $x$ & $\begin{array}{l}\text { Color aerial } \\
\text { photograph }\end{array}$ & $\begin{array}{c}\text { (Garden et } \\
\text { al., 2001) }\end{array}$ \\
\hline $\begin{array}{l}\text { Southeast } \\
\text { Utah, USA }\end{array}$ & $\begin{array}{l}\text { Paleo-fluid } \\
\text { migration } \\
\text { footprints }\end{array}$ & $\begin{array}{c}\text { Navajo } \\
\text { sandstone }\end{array}$ & $\begin{array}{l}\text { - Iron cementation } \\
\text { - Iron bleaching }\end{array}$ & $x$ & $\begin{array}{l}\text { Color aerial } \\
\text { photograph }\end{array}$ & $\left(\begin{array}{c}\text { (Nielson et } \\
\text { al., 2014) }\end{array}\right.$ \\
\hline $\begin{array}{l}\text { Zion NP; } \\
\text { Utah, USA }\end{array}$ & $\begin{array}{l}\text { Remnants of } \\
\text { reducing fluid }\end{array}$ & $\begin{array}{c}\text { Navajo } \\
\text { sandstone }\end{array}$ & $\begin{array}{l}\text { - Iron bleaching } \\
\text { - Iron enrichment }\end{array}$ & $x$ & & $\begin{array}{l}\text { (Nielsen et } \\
\text { al., 2009) }\end{array}$ \\
\hline $\begin{array}{l}\text { Southern } \\
\text { Utah, USA }\end{array}$ & $\begin{array}{l}\text { Exhumed } \\
\text { reservoir }\end{array}$ & $\begin{array}{c}\text { Navajo } \\
\text { sandstone }\end{array}$ & $\begin{array}{l}\text { - Secondary iron oxides } \\
\text { - Late calcite cement (+ dolomite) } \\
\text { - kaolinite \& illite }\end{array}$ & $x$ & Landsat ETM & $\begin{array}{l}\text { (Beitler et } \\
\text { al., 2005) }\end{array}$ \\
\hline $\begin{array}{l}\text { Kaibab, Utah, } \\
\text { USA }\end{array}$ & $\begin{array}{l}\text { Exhumed } \\
\text { reservoir }\end{array}$ & $\begin{array}{c}\text { Navajo } \\
\text { sandstone }\end{array}$ & $\begin{array}{l}\text { - Secondary hematite cement } \\
\text { - Calcite } \\
\text { - Illite and kaolinite }\end{array}$ & $x$ & - & $\begin{array}{l}\text { (Parry et } \\
\text { al., 2004) }\end{array}$ \\
\hline $\begin{array}{l}\text { Elaterite } \\
\text { Basin, Utah, } \\
\text { USA }\end{array}$ & $\begin{array}{l}\text { Exhumed } \\
\text { reservoir }\end{array}$ & $\begin{array}{l}\text { Permian } \\
\text { White Rim } \\
\text { sandstone }\end{array}$ & $\begin{array}{l}\text { - Bleaching of red beds } \\
\text { - Secondary diffused or concretionary iron } \\
\text { - Pseudomorphs of pyrite } \\
\text { - Calcite precipitation } \\
\text { - Illite and kaolinite }\end{array}$ & $x$ & $\begin{array}{l}\text { GPS, geologic } \\
\text { map }\end{array}$ & $\begin{array}{c}\text { (Gorenc } \\
\text { and Chan, } \\
2015)\end{array}$ \\
\hline $\begin{array}{l}\text { Colorado } \\
\text { Plateau, USA }\end{array}$ & $\begin{array}{l}\text { Exhumed } \\
\text { reservoir }\end{array}$ & $\begin{array}{c}\text { Glen } \\
\text { Canyon } \\
\text { sandstones }\end{array}$ & $\begin{array}{l}\text { - Bleaching of iron oxides } \\
\text { - Clays }\end{array}$ & $x$ & Landsat ETM & $\begin{array}{l}\text { (Beitler et } \\
\text { al., 2003) }\end{array}$ \\
\hline $\begin{array}{l}\text { Onshore } \\
\text { wells, }\end{array}$ & Unknown & $\begin{array}{c}\text { Skagerrak } \\
\text { Fm. }\end{array}$ & $\begin{array}{l}\text { - Dolomite, anhydrite } \\
\text { - Mixed-layer illite/smectite, kaolinite }\end{array}$ & - & - & $\begin{array}{l}\text { (Weibel, } \\
\text { 1998) }\end{array}$ \\
\hline
\end{tabular}




\begin{tabular}{|c|c|c|c|c|c|c|}
\hline Denmark & & $\begin{array}{l}\text { (Arkosic red } \\
\text { sandstone) }\end{array}$ & $\begin{array}{l}\text { - Chlorite (Mg-rich in red and Fe-rich in whitish parts) } \\
\text { - Pyrite }\end{array}$ & & & \\
\hline $\begin{array}{l}\text { O'Neill } \\
\text { forebay } \\
\text { CA, USA }\end{array}$ & $\begin{array}{c}\text { Gas } \\
\text { microseepage } \\
?\end{array}$ & $\begin{array}{l}\text { Pleistocene } \\
\text { Corcoran } \\
\text { clay }\end{array}$ & $\begin{array}{l}\text { - Alunite, jarosite, and gypsum } \\
\text { - pyrite and iron sulfates } \\
\text { - Native sulfur and Acidic } \mathrm{pH}\end{array}$ & - & - & $\begin{array}{c}\text { (Prokopovi } \\
\text { ch et al., } \\
1971)\end{array}$ \\
\hline $\begin{array}{l}\text { San Rafael } \\
\text { Swell, UT, } \\
\text { USA }\end{array}$ & $\begin{array}{l}\text { Roll front of a } \\
\mathrm{CO}_{2} \text { reservoir }\end{array}$ & $\begin{array}{l}\text { Navajo } \\
\text { sandstone }\end{array}$ & $\begin{array}{l}\text { - (Oxyhdr) oxides (lepidocrocite/goethite/hematite) } \\
\text { - Clays } \\
\text { - Carbonate cementation (dolomite) }\end{array}$ & $x$ & - & $\begin{array}{l}\text { (Potter- } \\
\text { Mclntyre } \\
\text { et al., } \\
\text { 2013) }\end{array}$ \\
\hline $\begin{array}{l}\text { Green River, } \\
\text { Utah, USA }\end{array}$ & $\begin{array}{l}\text { Exhumed } \\
\text { natural } \mathrm{CO}_{2}\end{array}$ & $\begin{array}{l}\text { Entrada } \\
\text { sandstone }\end{array}$ & $\begin{array}{l}\text { - Bleaching } \\
\text { - Pore-filling calcite, dolomite, ferroan dolomite } \\
\text { - Gypsum veins } \\
\text { - Illite-smectite }\end{array}$ & $x$ & - & $\begin{array}{l}\text { (Wigley et } \\
\text { al., 2012) }\end{array}$ \\
\hline
\end{tabular}

Aside from exhumed $\mathrm{HC}$ reservoirs, the array of secondary mineralogy occurring within currently active oil and gas reservoirs and oil-sand deposits indeed indicate similar trends in diagenetic alteration (Cloutis et al., 1995; Matthews, 1986; Parry et al., 2009). A case in point is the Athabasca's oil-sands in which the bitumen is accompanied by clays (i.e. kaolinite, illite, montmorillonite, and chlorite), Fe-carbonates (siderite), and pyrite (Cloutis et al., 1995). This trend, however, does not imply that all the alteration/bleaching patterns observed in sedimentary environments arise from thermogenic $\mathrm{HCs}$; because the circulation of other reducing agents such as biogenic methane, organic acids, $\mathrm{CO}_{2}$, and $\mathrm{H}_{2} \mathrm{~S}$ can give rise to similar alteration arrays (Chan et al., 2000; Nielsen et al., 2009). To denote this analogy, we included two case histories on $\mathrm{CO}_{2}$-induced transformations in Table 7. This underlines the significance for novel mineralogical indicators to distinguish the two phenomena apart. The subject is discussed in sections 6-2-2 and 6-2-6.

\section{Demonstration datasets}

Within this article, examples of remote sensing data over two study areas with distinctive macro-, and microseepage systems are presented as illustrative products. The first area located in the Ventura Basin (CA, USA) hosts a typical oil seepage system (e.g. (Ellis et al., 2001; Prelat et al., 2013; van Der Meer et al., 2002)) among outcrops of sandstone, conglomerate, mudstone, and shale units belonging to Pico, Sisquoc, and Monterey Formations (Fig. 5b). The data covering this test site were collected on August 26, 2014, during the HyspIRI Preparatory Campaign by AVIRIS instrument at an altitude of $20 \mathrm{~km}$ and a GSD of $15.9 \mathrm{~m}$ (Fig. 5a). The georectified reflectance dataset was acquired from the AVIRIS webpage and then was spectrally processed by a combination of matched filtering (MF) and logical operators designed to track the diagnostic absorption features of petroleum at 2300 and $1700 \mathrm{~nm}$. The resulting anomaly map is presented in Fig. 5c. Selected anomalies namely Seep-1, Seep-4, Roof-1, and Plastic Paint were verified spectrally (Fig. 5d) and visually (Fig. 5e-5h) using respectively the spectral content of the imagery and high-resolution satellite imagery.

The second study area is located in Northern Tucano basin in Bahia, Brazil. The area is ruled by a semiarid climate and consequently, the bedrock is fully exposed to satellite sensors (Fig. 6c). The main rock units in the area are conglomerate, sandstone, shale, and silicified limestone that collectively belong to Tona Sequence and Marizal Formation (Fig. 6a). Possible HC accumulation in a structural trap in this area is accompanied by marginal soil-gas anomalies (Almeida-Filho et al., 2002; Lammoglia et al., 2008). The soil-gas data were employed to assess the remotely-sensed spectral anomalies. For this aim, ethane to pentane readings were firstly normalized by dividing them to their maximums and then summed up to represent the total $\mathrm{HC}$ content of gasses heavier than methane $\left(\sum C_{2}-C_{5}\right)$. The data were subsequently interpolated using 
the Empirical Bayesian Kriging technique available in ArcMap 10.3 software (www.esri.com) and illustrated in color-coded raster format (Fig. 6b). The datasets from Landsat-OLI (Fig. 6c) and ASTER multispectral instruments acquired respectively on 2016/10/06 and 2006/11/04 were used to map the intrinsic alterations within this microseepage system. In the spectral processing stage, we mapped the distribution of clays, ferrous iron (e.g. Fe-carbonates, chlorite, etc.), and ferric iron oxides/oxyhydroxides (e.g. goethite, hematite \pm ferrihydrite) by employing a partial unmixing technique and proper endmembers derived manually from ASTER-SWIR and Landsat imageries. The resultant maps are shown in Fig. 6d-6f.
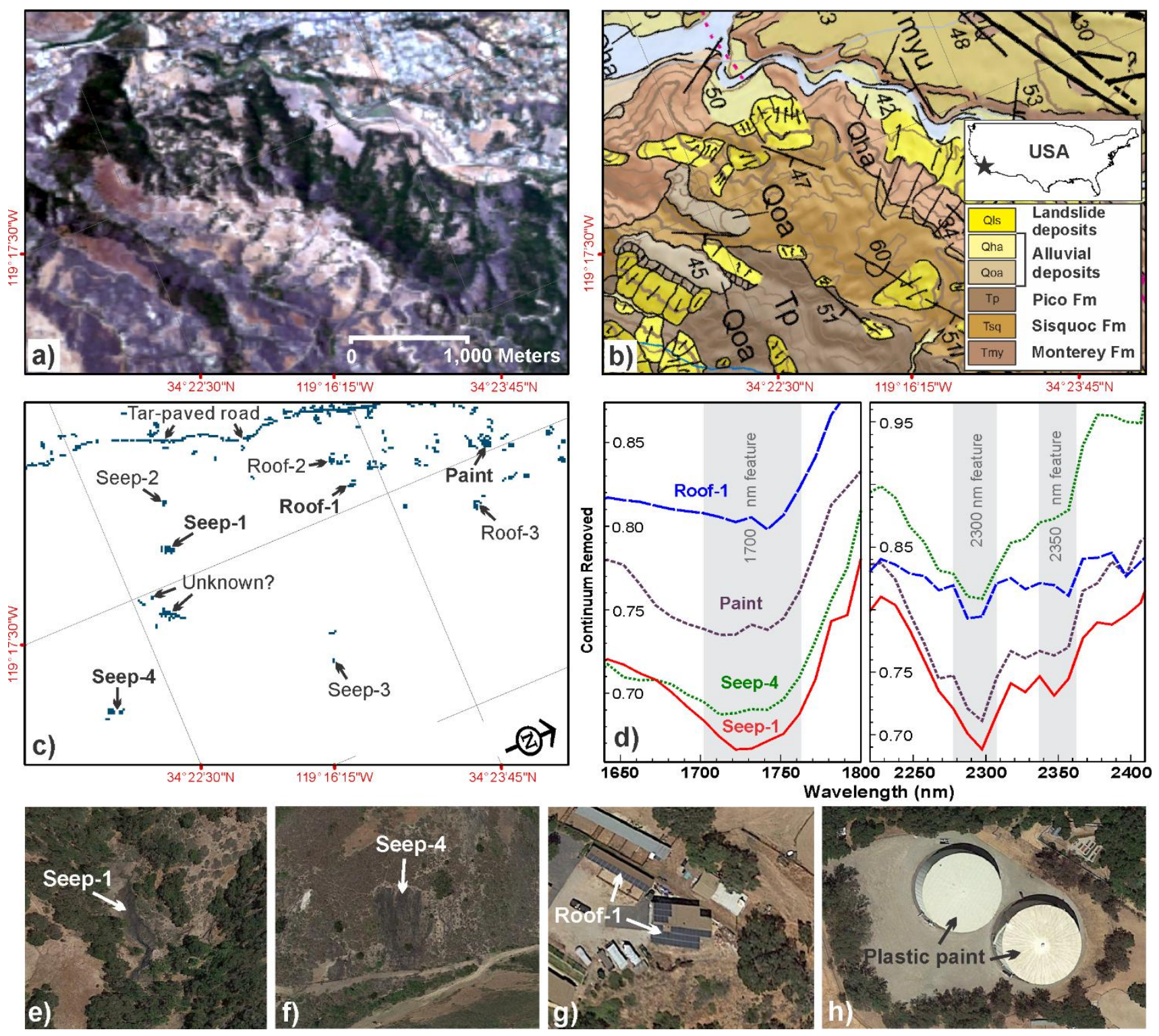

Fig 5. Macroseepage demonstration case study located in the Ventura Basin, California, USA. a) natural color composite of the AVIRIS data. b) geologic units of the area adapted from 1:100,000-scale geologic map of the Santa Barbara $30^{\prime} \times 60^{\prime}$ quadrangle compiled by Gutierrez (2008). The location of the study area is shown in the inset map. c) anomaly map extracted from hyperspectral data following spectral analysis. Arrows indicate the location of the oil seeps and other spectrally similar petroleum-bearing compounds. d) continuum-removed reflectance spectra of four typical anomalies mapped in (c) achieved by averaging the relevant pixels in the hypercube data. The two major absorption features of petroleum centered at $1700 \mathrm{~nm}$ and $2300 \mathrm{~nm}$ are shown as gray columns in the left and right panels, respectively. e-h) high-resolution satellite imagery (GSD of $\sim 1 \mathrm{~m}$ ) of selected anomalies shown in (c) and (d), including oil seep-1 (e) located at coordinates $34^{\circ} 22^{\prime} 43.7^{\prime \prime} \mathrm{N}$ and $119^{\circ} 17^{\prime} 40.5^{\prime \prime} \mathrm{W}$; oil seep-4 (f) located at $34^{\circ} 22^{\prime} 13.2^{\prime \prime} \mathrm{N}$ and $119^{\circ} 16^{\prime} 59.5^{\prime \prime} \mathrm{W}$, roof-1 (g), and the plastic paint (?) of twin tanks (h). Source: Google Earth, imagery acquired in 1st May 1, 2015. 

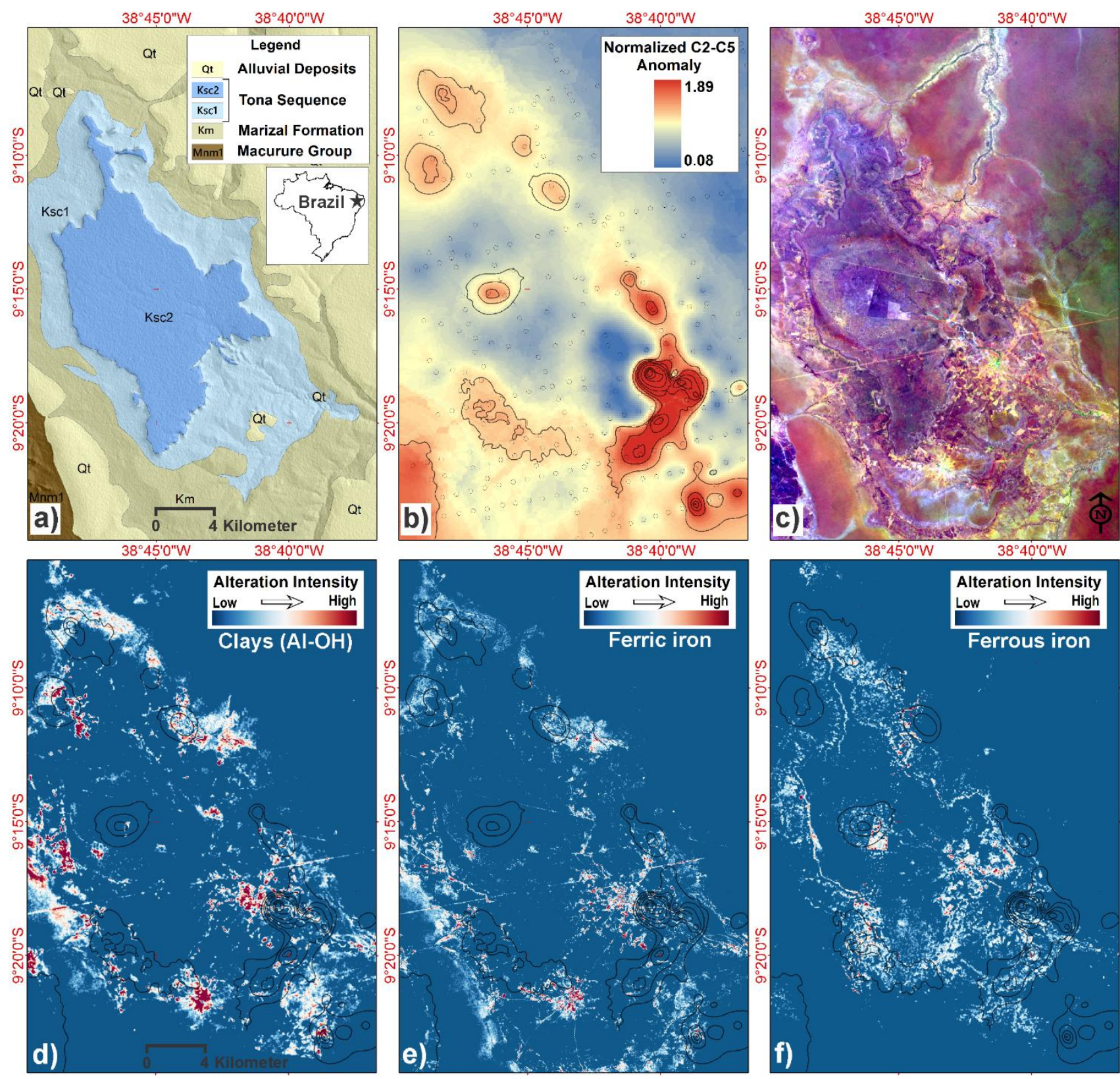

Fig 6. Microseepage demonstration case study located in the Northern Tucano basin, Bahia, Brazil. a). generalized geologic map of the area. The location of the study area is shown in the inset map. b) Interpolated soil-gas anomaly map calculated from summing the normalized $\mathrm{C}_{2}-\mathrm{C}_{5}$ readings. The sampling sites are shown by open circles. c) false color composite $(R=b 7(2201), G=$ $b 5(864), B=b 2(482 \mathrm{~nm}))$ imagery of the Landsat-OLI data. d) relative abundance of clay alteration (including kaolinite, illite, and/or smectites) mapped using ASTER data. e) relative abundance of ferric iron minerals (e.g. goethite and hematite) extracted from Landsat data. f) relative abundance of ferrous iron minerals (e.g. Fe-carbonate, chlorite, and so forth) extracted from ASTER data. The overlaid contour lines in (d) to (f) were achieved by setting a threshold of 0.33 for the map shown in (b). The mineral abundances were calculated relative to image-derived endmembers.

\section{Discussion}

\subsection{Direct sensing methods}

The capability of remote sensing to detect and map oil and gas seeps can be used to: (i) screen frontier basins for any seepage manifestations, (ii) record the size, type, and possibly the likely replenishment of a 
leakage; (iii) update/map the seepage activity in mature/productive basins; (iv) assess the instant and longterm flux of the known seeps; and (v) compile global thematic databases for natural HC seeps.

Gas-plume sensing capability depends heavily on the seepage flux, wind speed, the specification of the deployed sensor (i.e. the spectral resolution and signal-to-noise ratio; SNR), and background cover, with spectrally uniform images being more advantageous relative to spectrally and thermally heterogeneous scenes (Frankenberg et al., 2016; Leifer et al., 2012b; Thorpe et al., 2014). Unlike the SWIR range, which is dependent on surface albedo, sensors in the LWIR range rely merely on the thermal emission and thermal contrast between ground and target gas. Hence, gas sensing in the LWIR could be more robust over a wider variety of land covers (Hulley et al., 2016). Indeed, simultaneous SWIR-LWIR data acquisition is required to investigate this notion. In both ranges, however, high spectral resolution data are required to distinguish the signatures of trace gas from interfering components (Hulley et al., 2016; Leifer et al., 2012b; Thorpe et al., 2016). Gas-plume sensing case studies thus far have been confined to methane detection that is highly significant for partitioning the sources of greenhouse gasses; albeit for oil and gas exploration, ethane $\left(\mathrm{C}_{2+}\right)$ constitutes a better exploration indicator (Jones and Drozd, 1983). Because methane emissions from geologic sources can incorporate $2-6 \%$ ethane on average (Etiope and Ciccioli, 2009), the recorded signal over natural plumes can be the overlap of methane and ethane signatures. The possibility of tracing ethane across geologic plumes is yet to be explored.

On the other hand, oil seepage detection is a function of seepage areal coverage, flowing rate, petroleum type, and geologic context, to name a few. Typically, petroleum sensing is bound to diagnostic absorption features centered at 1700 and $2300 \mathrm{~nm}$ (Fig. 5d), for the features centered at 1200 and $1400 \mathrm{~nm}$ are respectively uncommon and interfered by atmospheric water vapor. Within a seepage indicator, crude oil tends to mix physically with its background; thus, both of the absorption features are consistently modified by the mixed material(s). The $1700 \mathrm{~nm}$ feature is only overlapped by an absorption feature of sulfates (i.e. gypsum and alunite) centered at $\sim 1750 \mathrm{~nm}$ (Fig. 7). However, in vegetated areas, the feature could be confused with non-photosynthetic vegetation. Furthermore, due to its proximity to water vapor band at $1900 \mathrm{~nm}$, the long-wave side of the feature is prone to residual atmospheric contamination (Kokaly et al., 2013).

The feature at $2300 \mathrm{~nm}$ is particularly noticeable and persistent, albeit it is susceptible to be overlapped by clays and carbonates (Scafutto and Souza Filho, 2016) (Fig. 7). Although the feature centered at $1200 \mathrm{~nm}$ has been infrequently used for HC delineation (Clark et al., 2010), investigations have demonstrated its usefulness in differentiating false anomalies arising from plastic contaminations (Winkelmann, 2005). In the case study shown in Fig. 5, the limitations imposed by SNR of the data (likely due to poor atmospheric correction) hampered the efforts to accomplish this goal. However, marked differences in the $1700 \mathrm{~nm}$ feature of oil seeps and artificial compounds were noticed (left panel in Fig. $5 \mathrm{~d}$ ).

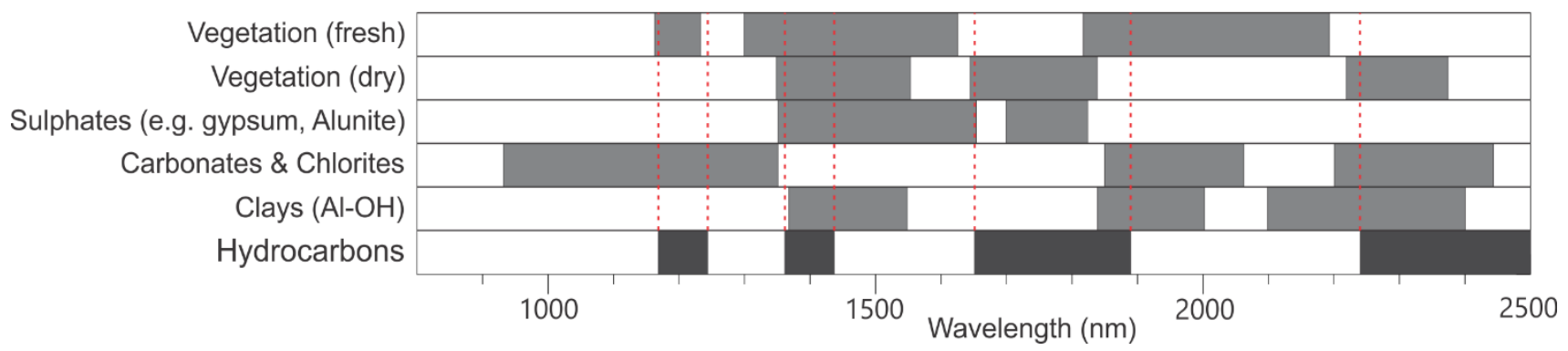


Fig 7. Spectral signature diagram of typical oil (obtained from Fig. 2) contrasted to absorption features of common sedimentary minerals as well as dry/fresh vegetation. The width of black bars indicate the relative widths of relevant absorption features.

To detect oil seeps effectively, a sensor should be equipped with proper spectral bands to resolve the features at 2300 and/or $1700 \mathrm{~nm}$ wavelengths (Fig. 5). For this reason, published case studies are confined to airborne systems (Table 4). Alternative spaceborne platform for this aim is due to be attained; albeit trials with WV-3 data has indicated the potentials of the sensor for this aim (Asadzadeh and Souza Filho, 2016a).

In terms of processing methodology, techniques like anomaly detection can be inadequate for $\mathrm{HC}$ mapping (Winkelmann, 2005). Instead, knowledge-based approaches (Asadzadeh and Souza Filho, 2016b) or hybrid methodologies that incorporate spectral-based decision-making system with feature tracking have been proven to yield superior results (Kokaly et al., 2013; Prelat et al., 2013) (see also the anomaly map in Fig. 5c).

The small size of oil seeps implies that high spatial resolution data (sub-decameter; Table 4) would be required to characterize them. The inclusion of alteration halos associated with a good portion of the seeps (i.e. miniseeps described in Table 6), however, can be used to facilitate their detection. A basic algorithm of this kind is developed by van der Werff (2006).

We should emphasize that petroleum detection includes but is not limited to the SWIR range. Crude oil is demonstrated to retain distinctive absorption features in the LWIR indeed (Lammoglia and Souza Filho, 2011). Nevertheless, due to the lack of a typical spectral library for crude oil diversity (and similar organic and petrochemical compounds) between 2.5-15 $\mu \mathrm{m}$ and also limited number of hyperspectral sensors operating in this range, the potential applications of the LWIR for oil seepage detection has remained largely unknown.

\subsubsection{The detection limit of $\mathrm{HCs}$}

The detection limit in remote sensing is defined as the smallest areal extent of a target (here a seep) within a pixel detectable spectrally. The detection limit for the case studies in Table 4 was reported to vary between 2.5 to $25 \%$ of a pixel. However, due to limited studies, this finding cannot be conclusive. In soil sciences, by comparison, the detection limit for the Total Petroleum Hydrocarbon (TPH) yielded from infrared spectroscopy varies from a few percent to a few hundredths of a percent (Chakraborty et al., 2015; Chakraborty et al., 2010; Correa Pabón and Souza Filho, 2016; Okparanma et al., 2014; Schwartz et al., 2013; Stallard et al., 1996). Correspondingly, the Total Bitumen Content (TBC) of oil-sand ores at around $1 \%$ wt. is routinely estimated via close-range spectroscopy (Lyder et al., 2010; Shaw and Kratochvil, 1990).

Some examinations have demonstrated that oil detection in the environment has a dependency upon physical and chemical properties of the soil matrix. For example, oil in siliciclastic or coarse-grained soils was easier to detect (at lower concentration levels) than in calcareous or fine-grained soils. The detection limit was also observed to be a function of API gravity, with heavier oils setting easier targets for spectral detection. The latter was likely due to the fact that heavier oils impregnated only the surface of the grains without being absorbed by porous media (Scafutto et al., 2016).

Overall, close-range investigations indicate that oil in the environment could be detected spectrally in very low quantities, albeit this finding cannot be directly linked to seepage reconnaissance. Apart from the fact that many studies were carried out by HCs other than crude oil, the experimental settings incorporated 
instant physical mixing of oil with some predetermined soils in volumetric fashion, whereas seeps and their pollution counterparts appear as areally (or combined areally and volumetrically) mixed targets in the imagery. This could complicate the underlying principles of mixture analysis and affect the smallest extent of oiled surfaces detectable spectrally. Further research is required to appreciate the possibility of upscaling the lab findings to remote sensing imagery and figure out the practical detection limit of far-range systems.

Concerning the methane detection limit, in controlled release experiments, thermal sensing technology was shown to detect methane fluxes as small as $4-5 \mathrm{~kg} / \mathrm{h}$ (Hulley et al., 2016; Tratt et al., 2014), whereas in the SWIR range, this level, depending on wind speed, was varied between $2-5 \mathrm{~kg} / \mathrm{h}$ (Frankenberg et al., 2016; Thorpe et al., 2016). Based on field measurements, the leakage flux for high-level microseepage is $>50$ $\mathrm{mg} \mathrm{m}^{-2} \mathrm{~d}^{-1}$ that increases to an average of $2 \times 10^{4} \mathrm{gd}^{-1}(\sim 1 \mathrm{~kg} / \mathrm{h})$ for macroseeps (Etiope, 2015). It means that current technology can detect macroseeps that are emitting average to high levels of gaseous HCs, albeit it is not appropriate to detect microseepage-level fluxes. Above all, available technology can only afford to detect distinct point sources. For areal (diffused) sources, which are most common in petroliferous areas, a sensor with higher sensitivity (lower detection limit) would be required.

\subsubsection{Petroleum quantification}

In the seepage context, spectral techniques can be employed to quantify the dimension of a seep and possibly the amount of HCs in the environment. In close-range experiments, the TPH is assessed by a predictive model initially derived from a suite of training samples using the Partial Least Squares Regression (PLSR) technique (e.g. (Okparanma et al., 2014)). This approach, however, is not appealing for image data mostly because it is case dependent and requires auxiliary data. An alternative approach is to use the continuum band-depth of HC's diagnostic absorption features for abundance quantification in relative or absolute terms (Asadzadeh and Souza Filho, 2016b).

The few studies on the use of 1700 and $2300 \mathrm{~nm}$ features have indicated the merits of spectral parameters, especially the 'depth' and 'area' of the absorption, for HC quantification (Correa Pabón and Souza Filho, 2016; Scafutto et al., 2016). Nonetheless, the significance of each feature for this aim has been a matter of debate. For instance, in an analysis based on the correlation coefficients of different wavelet scales with TBC, it was demonstrated that the feature at $2300 \mathrm{~nm}$ appears correlated in several scales (from 1-7), whereas the $1700 \mathrm{~nm}$ feature shows correlation with only two (4 and 5) scales (Lyder et al., 2010). Based on this persistency, it was concluded that the $2300 \mathrm{~nm}$ feature is more successful in delineating the $\mathrm{HC}$ content of oil-sand samples. In the demonstration case study (Fig. 5a), the depth of these features was calculated after continuum removal, but due to the low spatial resolution of the imagery $(\sim 16 \mathrm{~m})$, it was difficult to identify a meaningful trend and thus was discarded.

Even though the depth of the feature at $1700 \mathrm{~nm}$ was shown to be linearly correlated to specific levels of HCs (Correa Pabón and Souza Filho, 2016), in general, the correlation between HC content and the noted spectral parameters tends to exhibit nonlinear behaviors. Experiments with the incremental level of oil also showed that in a certain level, the soil sample becomes saturated, thereby breaking the correlative relationship. Identical to the detection limit, the saturation point was proved to be a function of oil type and intrinsic soil characters (Scafutto et al., 2016). By comparison, the feature at $1700 \mathrm{~nm}$ reaches to saturation at higher $\mathrm{HC}$ levels than the $2300 \mathrm{~nm}$ feature. A related point to consider is that the saturation level was observed in volumetric experiments and it is not known as if it would be the case for areal mixture. 
In conclusion, such observations imply that potential (nonlinear) predictive models for HC quantification from spectral data would be valid between the 'detection limit' and 'saturation point'. Further research is required to better understand the behavior of noted spectral parameters against different levels of petroleum in a sample/pixel.

\subsubsection{Petroleum characterization}

Infrared spectroscopy is unlikely to be able to identify petroleum types (e.g. kerosene vs. diesel), particularly where it is mixed with soil particles. Instead, it was proved to be effective in characterizing oil types based on API gravity (a measure of how heavy liquid HC is, compared to water) and SARA (Saturate, Aromatic, Resin, and Asphalten) fractionation index (Aske et al., 2001; Lammoglia and Souza Filho, 2011). The approach incorporates a mathematical model experimentally constructed using a sort of correlation algorithm (e.g. PLSR) to predict the noted chemical properties. Although the methodologies have been successful in characterizing the API and SARA index of given oils in the lab, so far, only the API has been remotely assessed (mapped) in the marine environment using multispectral ASTER data (Lammoglia and Souza Filho, 2012) and similar onshore demonstration is yet to be provided. The latter, on the other hand, has remained a laboratory experiment and not attempted via image data. Although the spectroscopic-based API model was developed using a specific collection of crude oils ( ${ }^{\circ} \mathrm{API}$ between 14-40), the underlying principle can be extrapolated to other compounds like fuel HCs.

\subsection{Indirect RS methods}

In oil and gas exploration, remote sensing could be utilized to screen a frontier basin for signs of a petroleum system, generate new exploration prospects, and evaluate the existence of microseepage anomaly over previously defined exploration leads (van Der Meer et al., 2002). Alike other surveying methods relying on microseepage theory, this approach neither can indicate the depth of an accumulation nor predict the economic success of wildcat drilling. In contrast to the soil-gas method that can directly verify the presence of HC, this technique should be applied to 'infer' the HC presence in a trap (Jones and Drozd, 1983; Schumacher, 1999). This technique shall be effective in dealing with bedrocks covered by sparse vegetation (i.e. $<30 \%$ ). Above this threshold, the use of mineralogical signature becomes limited and one should consider other realms of remote sensing including geobotany for microseepage detection. Obviously, spectral technique merely measure the reflected/emitted energy from topmost parts of the surface and is unable to penetrate into it.

A close inspection of the documented case studies in Table 6 reveals a large gap between the true potentials of remote sensing and the achieved results. Here, we highlight several of the shortcomings and subsequently give some guidelines for further case studies in the future.

\subsubsection{The shortcomings of the case studies}

Unfortunately, the number of well-documented publications on the subject, as summarized in this paper, are not considerable ( $<30$ cases), while a large proportion of them was dedicated to miniseeps that are indications of macroseeps rather than microseepage systems. A typical remote sensing study in local/regional scale should be augmented by detailed fieldwork and lab analysis. Nevertheless, a look at Table 6 indicates that the number of collected samples in each case study is inadequate and the sampling schemes are mostly disordered (Figs. 4d-4e), resulting in poor relationships between sampling sites and 
remote sensing anomalies. The benefits of detailed outcrop studies as a missing link in such investigations is best exemplified by the outlined studies in Table 7 .

Because target minerals of a microseepage system are already common in sedimentary basins, it is important to draw the comparison in a relative term. In other words, a microseepage-affected zone should be compared to a 'reference area' inside the intact (unaffected) extent of the same lithologic unit. This effective strategy, which is commonly used to determine the background in geophysical and geochemical surveys (Abrams, 2005; Saunders et al., 1991), has been adopted by very few investigations (Fig. 4c). In addition, due to basic processing algorithms (Fig. 3o), the approach was only able to separate $\mathrm{HC}$ affected zones (anomaly) from their surroundings (background), without delineating individual minerals of the assemblage (Fig. 4a). To accomplish this objective and complement the results, spectroscopy (in situ or in the lab) could be used, but in the reviewed cases, this capacity has been rather underutilized. Spectroscopy not only can facilitate the identification of minerals, but can also be utilized to characterize slight compositional changes present in clays, carbonates, iron oxides/oxyhydroxides, and sulfates with reasonable accuracy.

A couple of studies have already shown that microseepage-induced alterations could be associated with spatial zoning. For example, some comprehensive fieldworks denoted that an extensively bleached area in red-beds surrounds an intense carbonate cementation zone along with pyrite patches occurring over productive limits of the reservoir (Al Shaieb et al., 1994). Despite the capability of image data to reveal possible spatial patterns of alterations, due to the scarcity of similarly detailed accounts, the zoning within microseepage systems has remained largely unknown. Furthermore, the spatial relationships between surface mineralogy relative to the 3D architecture of a reservoir are poorly understood. The only clue in this regard provided by measurements of magnetic susceptibility of exploration drill cuttings demonstrated that authigenic magnetic minerals (i.e. maghemite) are mostly distributed in depth range of $60-600 \mathrm{~m}$ above $\mathrm{HC}$ reservoirs (Foote, 1992; Foote, 1996; LeSchack and Van Alstine, 2002). Comparative studies between remote sensing anomalies and seismic data (e.g. (Shi et al., 2012)) or drill cuttings can be an asset for this aim.

All the outlined case studies investigate charged (productive/prospective) plays and none is focused on failure cases or unproven/dry prospects to have a fair unbiased estimate for false-positive and false-negative anomalies (see the next section). From a mineralogical point of view, it is entirely unclear if secondary anomalies are present where a trap retains no accumulation, or if the features are unique to already charged traps. Based on exhumed reservoir case studies, however, it seems that several (but not all) of the classic characteristics (including bleaching) are shared between fossil (paleo) and active microseepage systems. Additionally, the characters of underlying traps including underpressured vs. overpressured reservoirs (given that pressure is the main drive for $\mathrm{HC}$ migration), stratigraphic vs. structural traps, and the sealing are not yet properly addressed.

The effect of microseepage on sediment covers (e.g., transported floodplain alluvium and loess) is not extensively evaluated in the literature. Although some have reported that transported covers (i.e. glacial drifts) can obscure the microseepage-induced radiometric signals (Price, 1996; Saunders and Terry, 1985), there are numerous other studies that denote it is not the case (e.g. (LeSchack, 1997)). The surficial nature of radiometric survey ( $<25 \mathrm{~cm}$ penetration depth) implies that secondary alterations can extend into a cover (provided a physically stable cover), and thus remote sensing approach should be able to detect the effects of microseepage in a similar manner. Some observations on the pace of mineralogical transformations can support such notion (see section 6-2-5). 
So far, the outlined case studies deal with nearly mono-lithologic sandstone units (Fig. 3e). Although clastic rocks and oxidized continental facies (red-beds) form at the latest depositional cycle of a sedimentary basin, they cannot represent the whole variety of the bedrocks present above $\mathrm{HC}$ accumulations. Sandstones at best account for around $25 \%$ of the sedimentary rocks (Boggs, 2009) and most likely cover the sedimentary basins with the same proportion. Nonetheless, a ubiquitous proportion of HC traps are either overlaid by sediments other than sandstones, are multi-lithologic in essence, or are concealed by recent sediment covers. To have a complete picture of the variations, studies shall be diverted towards more diverse lithologies exposed over HC accumulations. The sandstones themselves retain large within-group variations that are neither considered in remote sensing studies nor in the classic microseepage model (see also section 6-2-4).

\subsubsection{False-positive/negative anomalies}

While there is statistics for positive and negative geochemical anomalies above exploration targets (section 2-3), such data have not been compiled for mineralogical-based remote sensing studies (see also section 6-2-8). In a sedimentary basin, any false-positive anomaly should be regarded as important as true anomalies, because a cross-comparison between these two groups can provide insights about how to distinguish them spectrally. As discussed by Brown (2000), present (and past) surface geochemical anomalies and associated alterations may result from uneconomic petroleum accumulations or migration pathways. Whereas remote sensing cannot distinguish between active microseepage systems arising from uneconomic and economic accumulations, it can be potentially used to exclude some other sources of false-positive anomalies. Generally, the following sources of false-positive anomalies in sedimentary basins should be recognized: (i) anomalies arising from processes unrelated to $\mathrm{HC}$ accumulations; (ii) anomalies occurring above depleted traps that once retained an active microseepage system; and (iii) anomalies intrinsic to sedimentary units.

In the first group, a number of already known geological processes give rise to roughly similar alteration assemblages. For example, the calcite cement in several soil profiles may be 'caliche', which is an amorphous soil salt originating from near-surface processes (Price, 1996). Pedogenic processes linked to climate conditions can initiate redox zones and precipitate maghemite, thereby yielding characters that may bear a resemblance to microseepage effects (Klusman, 2002; Schumacher, 1996). Similar alterations can also arise from shallow gasses of biogenic rather than thermogenic sources (Schumacher, 1999). Moreover, the circulation of abnormal amounts of $\mathrm{H}_{2} \mathrm{~S}, \mathrm{CO}_{2}$, and organic acids in permeable sediments may develop signatures similar to microseepage-induced alterations (Parry et al., 2004; Schumacher, 1996) (see also Table 7).

As petroleum system implies, HCs are preserved in a trap if the sum of leakage or destruction is less than the petroleum charge; otherwise, a trap would be devoid of HCs (Hunt, 1996; Magoon and Beaumont, 1999). Therefore, paleo-microseepage systems over depleted traps would possibly leave mineralogical footprints that are similar to active systems. The best analogy where this phenomenon could be investigated is exhumed HC reservoirs cropping out throughout the Colorado Plateau (Table 7). This phenomenon substantiates that when the intensity of alteration is severe, the induced changes can survive through geologic time and emerge as false-positive anomalies in present-day surveys. Parallel outcrop studies also demonstrated that several episodes of fluid flow (meteoric and interstitial water and migrating HCs) could coincide to shape the final alteration facies (see Table 7). Finally, because the seeking diagenetic minerals (e.g. clays and carbonates) are intrinsically abundant in the background strata, occasionally, they could become a source of false-positive anomalies. Such anomalies are subject to inter-, and intra-unit variations. 
All of the abovementioned instances create ambiguity in remote sensing data interpretations. Whereas part of the ambiguity is intrinsic to the approach and unavoidable, we believe the other part could be avoided by applying efficient methodology, enhanced imaging tools, and above all, by promoting our understanding of the diagenetic changes. A case in point is the problem of alike background mineralogy that could be circumvented by setting a reference area off the affected zone for cross-comparison. The gained experience in mineral exploration implies that spectroscopic products including abundance, composition, and association of minerals derived from hyperspectral data may offer potentials for segregating real anomalies from false ones (see also section 6-2-6). In this regard, the authors believe that comparative studies between active microseepage systems and exhumed $\mathrm{HC}$ reservoirs could facilitate the development of mineralogical indicators to eliminate false-positive anomalies over fossil systems. Altogether, true anomalies typically are expected to cover vast areas and conform to regular spatial patterns (Fig. 6; see also section 6-2-7).

In the literature, there is no unbiased evaluation of remotely-sensed false-negative anomalies. To assess this parameter, the outcomes of this technique should be compared to geochemical data or evaluated by wildcat drilling indeed.

\subsubsection{Sensor obstacles}

Part of the reasons for the limited success of remote sensing in microseepage delineation is due to limitations imposed by multispectral sensors. In contrast to technological refinement in geochemical and geophysical analytical methods, the operational sensing capability of this approach has not witnessed dramatic advancements in the last decades. In Earth resource exploration, in particular, the only promotion in three decades has been the launch of ASTER multispectral instrument and more powerful hyperspectral technology has been pursued solely from airborne platforms. Newer satellite systems with better performances such as Sentinel-2 (2015) and WV-3 (2014), which could be potentially used for enhanced spectral mapping, were launched only recently. A leap that is due to revolutionize this discipline, however, is the arrival of spaceborne hyperspectral imaging systems like the EnMap satellite (http://www.enmap.org/). Because microseepage phenomena are bound to yield large targets, they would be fully resolved with moderate spatial resolution (i.e. $30 \mathrm{~m}$ ) of such instruments. In the case study shown in Fig. 6, the $30 \mathrm{~m}$ resolution of ASTER/Landsat data were enough to resolve the targets, however, due to a limited number of bands, the discrimination of mineral species was not straightforward.

Thus far, the majority of published studies for microseepage characterization are limited to the VNIRSWIR window and the literature is deficient in case studies using thermal infrared (LWIR) data. A suite of diagenetic changes in the chimney column such as silica enrichment (Almeida-Filho et al., 2002; Thompson et al., 1994) or feldspars depletion (alteration) are solely mappable via this dataset. The LWIR wavelength or an integrated multi-wavelength spectral analysis can also promote the mapping of aforementioned alterations with greater accuracy.

\subsubsection{The inadequacy of the microseepage model}

In remote sensing data analysis, the microseepage model is used to define target minerals and subsequently to attribute the mapped minerals to microseepage effects. We believe the inadequacy of the present model is causing considerable uncertainties in the interpretation of resultant maps because it fails to fully delineate the quantity, quality, and diversity of mineralogical components present in a system. The existing theory, which evolved during the 1970s and 80s principally to explain anomalous gas levels over HC 
accumulations, was ultimately formulated in 1990s to account for alteration facies in a general term (Saunders et al., 1999; Schumacher, 1996; Thompson et al., 1994). Unfortunately, this model simply assumes global identical features for the phenomenon without taking into consideration the effects of local environmental variables (i.e. climate, hydrogeology, erosion, and soil-forming processes) and geologic setting (i.e. lithology and stratigraphy of the near-surface units) in which the microseepage occurs.

Microseepage and hydrothermal processes are somehow comparable phenomena. In a hydrothermal system, magma, as a source of energy, triggers fluid circulations and oxidation-reduction reactions in the host rock, leading to wall-rock alterations and ore deposition (Pirajno, 2009). In both systems, fractures provide the conduits for fluid transportation and subsequent mineral deposition, with macroscopic fractures playing a critical role in the overall mass transfer. Using this analogy, one can expect a similar trend in the diversity of alteration products within a chimney column, of which the collection described by the present model representing only a fraction. We postulate that upon providing a bigger picture of the mineral diversity, they could be categorized into a series of descriptive models, each tailored for specific geological settings. Such a multiplicity would then facilitate spectral processing and likely would contribute towards more efficient $\mathrm{HC}$ exploration. Below we attempt to outline some aspects of the mineralogy not reflected in the existing model.

The accepted notion about diagenetic clay minerals is based on kaolinite enrichment, but as we discussed earlier (e.g. Fig. 3j), a suite of indicative clays consisting of illite, smectites, and chlorites can be expected above $\mathrm{HC}$ accumulations. For instance, in Fig. 6f, the ferrous mineral map is expected to incorporate chlorite as well. Moreover, sulfates constitute a key mineralogical signature that has been overlooked altogether in the classic microseepage model. Due to a steady influx of $\mathrm{O}_{2}$, the vadose zone above the water table is strongly oxidized, thus many of the reduced minerals, including pyrite and Fecarbonates, become unstable and eventually weather (partially or fully) to other more stable forms. The oxidation of pyrite, in particular, can trigger the formation of several metastable $\mathrm{pH}$-sensitive iron sulfates such as jarosite, copiapite, melanterite, and schwertmannite in the system. Subsequent oxidation of this array gives rise to a series of ferric iron oxyhydroxides in the diagenetic facies, including ferrihydrite, maghemite, lepidocrocite, goethite, and occasionally secondary hematite (Elwood Madden et al., 2004). Unlike pyrite, all the subsequent sulfates and oxides are spectrally active in the VNIR-SWIR windows, thus are potentially detectable remotely (Crowley et al., 2003). A case in point is the suite of Fe-sulfate and oxide minerals successfully mapped using AVIRIS data over the Dutton Basin Anticline (Staskowski et al., 2004). In a very arid environment, however, jarosite has reported to endure weathering and constitute a potential target over several microseepage-related facies (Bell et al., 2010; Elwood Madden et al., 2004; Everett et al., 2002; Perry and Kruse, 2010). Ultimately, secondary hematite cement formed due to severe and prolonged oxidation of initial ferrous minerals have been observed in several oil and gas fields (Donovan et al., 19791982; Kirkland et al., 1995; Segal et al., 1986). The weathering, in a similar manner, can be responsible for the absence of magnetic bodies (e.g. maghemite) in near-surface ( $<60 \mathrm{~m}$ on average) section of the strata as recorded by well cuttings measurements (Foote, 1992; Foote, 1996).

Although many have emphasized the combined role of $\mathrm{pH} / \mathrm{Eh}$ in diagenetic changes, the $\mathrm{pH}$ of the soils and sediments is not commonly reported (Fig. 4f) and its contribution in shaping the mineralogical facies is not fully addressed. This parameter is highly significant for it controls the mineralogy of clays and regulates the species of iron oxyhydroxides in the strata (Marrs and Paylor., 1987).

\subsubsection{Microseepage within time}


Microseepage anomaly, as scrutinized by soil-gas and microbial techniques, is a dynamic and rapid phenomenon that can appear/disappear within several months (Rice et al., 2002; Schumacher, 2000; Tedesco, 1999). By contrast, the pace of mineralogical changes over chimney column has not yet been realized, mainly because of the lack of systematic investigations and different transformation timescales. Some sporadic observations, however, have demonstrated that the pace could be very quick in geologic time. For instance, a study conducted over a gas-storage area revealed that anomalous magnetic susceptibility in soil appears in less than 24 yrs. (Saunders et al., 1991). Observations in sanitary landfills also have revealed that mineralogical transformation above a seeping methane source, which supposedly bears some resemblance to microseepage system, is rapid and happens in less than a decade or two (Ellwood and Burkart, 1996). In a quite exceptional case, more rapid transformation, in the order of several months, has been witnessed in a dam construction area (Prokopovich et al., 1971). Such rapid rate of changes conforms to our observations of soil bleaching by the gas flow in a controlled environment.

According to Saunders (1999), microseepage is a dynamic phenomenon and gradually moves from the center of the accumulation to the outer edges. When the gas anomaly merely shifts or becomes extinct altogether, the evidence of the leakage, including anomalous secondary minerals, are preserved and accumulated in the sediments as 'fossil' geochemical anomalies (Saunders et al., 1991). Due to the accumulative nature of such fossilized footprints within time, they typically constitute major targets for remote sensing, even though it can bring about false-positive anomalies over depleted traps. Subsequent weathering/erosion is likely to fade the footprints out, albeit profound authigenic changes, much like exhumed reservoirs (Table 7), would survive into the present time. As an example, the spectral anomaly yielded from satellite data over an oil field in Tennessee has been observed to diminish over 25 years of production (Perry, 2006). As stated earlier, novel mineralogical indicators should be quested to tell the active and fossil systems apart. A relevant point to consider is that the secondary cementation of the strata and the accumulative character of diagenetic changes are the likely reasons behind some disagreements between soil-gas and mineralogical anomalies (Klusman and Saeed, 1996). In the case study shown in Fig. 6, however, the soil-gas and alteration signatures maintain a close spatial relationship (e.g. Fig. 6d).

\subsubsection{The necessity for quantitative mineral maps}

In the literature on microseepage topic, it is uncommon to account quantitatively for mineralogical changes. A typical example is the 'bleaching' term regularly used to indicate the loss of ferric iron without attempting to quantify the original and final levels. Based on few reports available, anomalous terrains were recognized by an increase in clay content between two to five times; sulfide content between two to three times; and 45\% more total carbonates (Marrs and Paylor., 1987; Schumacher, 1996; Segal and Merin, 1989). In absolute term, such anomalies were characterized by a reduction in iron content from $2.9 \%$ in the periphery to just $1.0 \%$ in the center of the alteration; a concentration of up to $2.0 \%$ wt. kaolinite; the occurrence of $1.6-5.7 \%$ sulfides; and $40-50 \%$ added carbonate cement (Donovan et al., 1975; Kirkland et al., 1995; Schumacher, 1996).

In order to increase the efficiency of remote sensing for microseepage delineation, we recommend adopting a quantitative approach for spectral processing using methodologies embedded in either knowledge-based or data-driven approaches (Asadzadeh and Souza Filho, 2016b). For example, it is possible to achieve a semi-quantitative map for hematite, kaolinite, and calcite by calculating the depth of the diagnostic absorption features centered at $\sim 850, \sim 2200$, and $2340 \mathrm{~nm}$, respectively (e.g. Fig. $6 \mathrm{~d}-6 \mathrm{f}$ ). To 
eliminate the effect of background mineralogy and intra-unit variations, however, the interpretation shall be based upon the concept of 'reference area' introduced earlier.

The lack of quantitative maps has also hampered the efforts to establish relationships between alteration intensity/zoning and seeping activity over HC pools. To unravel such likely relationships, quantitative remote sensing results should be interpreted in the context of geochemical anomalies or wildcat drilling (see section 6-2-9).

Apart from abundance, spectral data can be used to map the variations in composition and crystallinity of selected minerals. A case in point is the capability of hyperspectral data to track the shift in the wavelength of absorption minimum at $\sim 900 \mathrm{~nm}$ to discriminate between different iron oxide/oxyhydroxide species, namely maghemite, goethite, hematite, and ferrihydrite. This unexplored capability has important implications for exploration, because maghemite, for instance, is known to be responsible for the bulk of micromagnetic anomalies detected by geophysical surveys (Foote, 1996; Foote, 2013; Holysh and Toth, 1996; Saunders et al., 1991). Moreover, due to the sensitivity of Fe-bearing minerals to the $\mathrm{pH}$ of the environment (Swayze et al., 2000), a map like the one noted could be used to predict the pH over microseepage systems. Similar spectral products could be developed to differentiate carbonate species (dolomite, siderite, ankerite, calcite, and rhodochrosite) using the wavelength of the absorption feature between $2320-2350 \mathrm{~nm}$ (Gaffey, 1987). Possible variations in the physicochemistry of clays comprising illitesmectites, chlorites, and kaolinite (see (Asadzadeh and Souza Filho, 2016b) for the details) has not been deeply investigated; albeit in the case of chlorite, some well data analysis has shown dominant occurrence of Fe-rich chlorite in bleached zones (Weibel, 1998). More research is required to uncover the full potential of such spectral products for microseepage exploration and indeed false-positive elimination.

\subsubsection{The shape of anomalies}

A proper understanding of the shape of the microseepage-induced anomalies and their compliance with underlying accumulation is critical to oil and gas exploration (Jones and Drozd, 1983; Xuejing, 1992). Because the overall shape of anomalies largely depends upon the employed prospecting tool, here we first overview the known shapes yielded from other exploration methods and then discuss the expected and observed anomalies from image data.

Soil-gas anomalies can have various shapes that in the order of importance are: (i) halo (annular/ring) pattern, in which anomalous readings are aligned over the edges of the underlying accumulation (Figs. 8a and Fig. 6b); (ii) apical (bell-shaped), in which an area of high values is surrounded by a low background (Fig. 8b); (iii) crescent type, which is found over accumulations trapped against a fault; and (iv) linear, which is observed over surface traces of faults, likely due to the effusion of HCs along fault planes (Duchscherer, 1980; Duchscherer, 1982; Horvitz, 1980; Price, 1986; Price, 1996; Xuejing, 1992).

The noted patterns may be either continuous or discontinuous (patchy/broken), consisting of several unconnected patches (Fig. 8 and Fig. 6b) over an accumulation (Xuejing, 1992). Typically, due to offsets between the location of an anomaly and the underlying reservoir, geochemical anomaly is unlikely to closely resemble the shape of the underlying reservoir (Brown, 2000; Holysh and Toth, 1996; Jones and Drozd, 1983; Richers et al., 1986; Saunders et al., 1999; Thrasher et al., 1996).

Likewise, (micro)magnetic anomalies over HC accumulations can have four different types: (i) double hump anomaly, corresponding to more intensive values on the edges; (ii) ripples, which is composed of high frequency signals superimposed on higher amplitude regional trend; (iii) positive anomaly that is composed 
of increased magnetism over the entire field; and (iv) negative anomaly due to pyrite precipitation (Eventov, 2000). Radiometric anomalies, on the other hand, are reported to occur either as halo or saddle-shaped (apical) forms over reservoirs (Fig. 1). Whereas geophysical anomalies are not highly accurate in outlining accumulations (Saunders et al., 1993b; Saunders et al., 1999), microbial prospecting is known to yield apical anomaly and coincide exactly with the extent of the field (Price, 1986; Price, 1996).

Overall, the 'halo anomaly' is acknowledged to be the most common form among exploration techniques (see Fig. 6). Several reasons have been given to explain the occurrence of this pattern including: (i) higher density of fractures over the edges of a structure, (ii) lower bacterial activity over the edges (relative to apical zone), (iii) conformity to gas-water and oil-water contact, and (iv) caprock clogging (Eventov, 2000; Horvitz, 1980; Price, 1986; Saunders et al., 1999). Based on numerical modeling, Brown (2000) concluded that the halo anomaly and irregular distribution of apical anomalies are mainly due to fracture distribution. The locality of such anomalies is believed to overlap with the areas of maximum stress in a structure (Eventov, 2000).

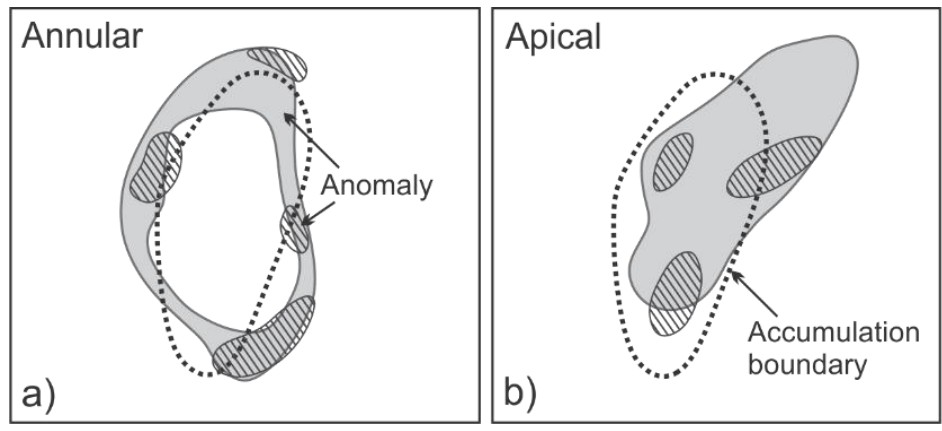

Fig. 8. Common surface geochemical anomalies over HC accumulations. a) Annular (halo). b) Apical. Solid and hatched fills indicate continuous and broken anomalies, respectively (Modified after (Xuejing, 1992)).

Based on field observations, a number of patterns for microseepage-induced mineralogy are already proposed as is illustrated in Fig. 1. However, in the absence of quantitative methodology, several of the illustrated forms have not been replicated via spectral data. Although some have pointed that the alteration anomalies over HC accumulations would exhibit a halo pattern (Klusman, 2002) (see also the case study in Fig. 6), in reality, the clogging of escaping routes (Saunders et al., 1999) along with the cumulative effect of alterations imply that the patterns should incorporate both apical and halo shapes. In other words, it could resemble an extended apical anomaly that depending on the mineralogy type will show positive or negative configuration. On the other hand, according to the descriptions provided in Table 6, it seems the 'disconnected apical' is the most likely pattern for mineralogical manifestations.

Saunders (1993a) has stated that "radiometric anomalies may be found only over portions of fields and thus it cannot be used to determine the production boundary accurately". In a similar way, it is rather unlikely that mineralogical indicators accurately conform to the shape of the underlying reservoir, albeit it is still a useful tool to locate microseepage systems arising from $\mathrm{HC}$ accumulations.

\subsubsection{The overall efficiency of remote sensing approach}


Owing to the fact that failure cases are absent in the reviewed case studies, an overall assessment of the efficiency of remote sensing in oil and gas exploration, much like geochemical method (e.g. (Schumacher, 2010)), is not feasible. Therefore in this section, we only overview the accounted success rates appeared in the literature.

In the early days of satellite data, it was reported that among the 57 hazy anomalies extracted from ERTS imagery in the Anadarko basin, 42 coincide with producing oil fields, corresponding to $73 \%$ coincidence (Short, 1977). In the era of Landsat, $75 \%$ of all mapped tonal anomalies (equivalent to 59 individual anomalies) were shown to be associated with economic HC plays (Feder, 1985). After drilling through 1177 geomorphic/tonal anomalies achieved from Landsat data in the Rocky Mountain, the average efficiency was assessed to be 54\% (Land, 1996), whereas in search of stratigraphic traps by an integrated exploration approach, success rates were reported to vary between $29-53 \%$ (Saunders et al., 1999). In an objective assessment of unconventional exploration methods, the performance of Landsat data in predicting the outcome of wildcat drilling over conventionally generated prospects was evaluated to be better than $75 \%$, which placed it among the best prospecting techniques evaluated (Calhoun, 1991).

In the era of 'spectral anomaly' extracted from ASTER data, $85 \%$ coincidence (18 out of 21 ) between spectral signatures and follow up fieldwork and geochemical sampling was recorded (Everett et al., 2002). In a recent study conducted in the Lake Albert basin in the East Africa Rift system, mineralogical anomalies due to $\mathrm{HC}$ migration were mapped using multisensory and multi-temporal satellite data (Table 6). Remarkably, spectral anomalies were detected in 18 out of 19 discovered (18 oil + 1 gas) fields, corresponding to 95\% success rate (Frassy et al., 2015). This study, however, neither provided the characteristics of the anomalies nor the results of probable field verifications.

Conceptually, the performance of spectral techniques could be compared to radiometric and magnetic methods, as both aim to detect microseepage-induced mineralogical indicators in a direct way. As an example, after investigating the radiometric data collected over 706 oil and gas fields in the US, it was discovered that $72.7 \%$ of them are associated with typical K and U anomalies (Saunders et al., 1993a). Based on other accounts, the radiometric and magnetic techniques were reported to correctly predict a production hole, respectively, at $59-85 \%$ and $58-75 \%$ of the times (Land, 1996; Potter II et al., 1996). With improvements in analytical instrumentations and processing methodology, higher success rates were witnessed by the noted techniques. A case in point is the statistics yielded from micromagnetic surveys indicating that over $80 \%$ of producing petroleum fields are associated with anomalous magnetic susceptibilities (Foote, 1992; Saunders et al., 1991; Wolleben and Greenlee, 2002). By using modern sensing technologies and processing methods, we can expect similar incremental trends in the performance of spectral remote sensing.

Microseepage-based prospecting tools are reportedly successful in predicting dry holes. For instance, 29 out of $30(96 \%)$ or 15 out of $19(79 \%)$ negative geochemical anomalies drilled in the field were ended up to be dry holes (Davidson, 2004). The geophysical methods, on the other hand, have been $70-90 \%$ successful, on average, in predicting a dry hole (Foote, 1992; Potter II et al., 1996). Similar calculation is yet to be carried out for remote sensing studies.

\subsubsection{Guidelines for future studies}

The study of microseepage phenomenon shall incorporate multi-scale data from fieldwork, lab analysis, and regional surveys. Outcrop investigation in this sequence, which includes but is not limited to sampling, maintains the essential link between regional and microscopic studies. To our knowledge, sampling along 
profiles is the best approach to intersect the bulk of variations induced by HC microseepage. We recommend establishing at least one reference area in unaffected (intact) part of the target unit(s) for cross-comparison of the induced changes. Certainly, this approach would require a priori knowledge about the stratigraphy of the units provided by a detailed geologic map. The overall number of samples collected from on/off zones should be large enough (typically $>30$ ) to reflect the concurrent effects of intra-unit and induced variations within a given area. Instead of simply differentiating anomaly from the background, it is more appropriate to characterize and quantify the mineralogy of anomalous zones (e.g. Fig. 6) by employing a proper spectral technique (Asadzadeh and Souza Filho, 2016b). This approach would help determine the shape of anomaly and reveal the spatial relationships between the mapped anomaly and possible subsurface pool.

The best supplementary analytical methods to corroborate remote sensing studies are those that provide fresh insights into the particularity of minerals in a system. Hence, beside the indispensable reflectance (and emittance) spectroscopy, the investigation should be rather complemented by XRD, optical microscopy, and Mössbaur spectroscopy. The latter has superiority in identifying the species of ferric/ferrous minerals in given samples. A deeper understanding of fluid-rock interactions, however, would be achieved when the studies are accompanied by stable isotope geochemistry.

Eventually, remote sensing data shall not be used in isolation. The integration of resultant maps into other non-seismic prospecting techniques not only can provide new insights into microseepage phenomenon, but can also add more value to the existing exploration data and improve the success of subsequent drilling (Prelat et al., 2013; Rice et al., 2016; Saunders et al., 1999).

\section{Seepages and the environment}

Although seeps have been a topic of concern to explorationists for a long time, until recently, there was little understanding of their role in carbon emission to the atmosphere. Based on recent assessments, macro-, and microseeps in total constitute the second most important sources of natural methane (and also ethane/propane) emissions to the air (Etiope and Ciccioli, 2009; Etiope and Klusman, 2010; Etiope et al., 2008). It has been estimated that between $21-36 \%$ of the geologic methane budget is emitted by seeps, of which microseepage-prone areas and macroseeps emit 10-25\% and $11 \%$, respectively (Etiope, 2015). The revised global budget via isotope data, however, suggested that methane emission from geologic sources is 60-110\% greater than current estimates (Schwietzke et al., 2016). The estimation of macroseepage flux has been relatively straightforward as a large portion of them are already known and monitored in the field. However, the global emission of microseepage is still uncertain because the calculations are based on averaging field contributions from identifiable homogeneous areas. The current flux is based on a database of 563 measurements in dry soils (Etiope and Klusman, 2010).

The potential microseepage-prone areas in the globe are estimated to be in the order of 3.5-4.2 million $\mathrm{Km}^{2}$ distributed within 937 petroliferous provinces or basins in 112 countries (Etiope and Klusman, 2010) that are approximately equivalent to $7 \%$ of the global dryland areas. Positive fluxes are typically a few to tens of $\mathrm{mg} \mathrm{m}^{-2} \mathrm{~d}^{-1}$ that can reach hundreds of $\mathrm{mg} \mathrm{m}^{-2} \mathrm{~d}^{-1}$ over widely tectonized zones (Etiope, 2015). Etiope and Klusman (2010) admitted that their calculation should be considered as a first spatial disaggregation of emission factors. They stated, "...the uncertainties in global emission estimates are mainly due to a poor knowledge of the dryland area of invisible microseepage". It is evident that all microseepage terrains occur within petroliferous provinces, but so far the actual microseepage areas have remained unknown (Etiope and Klusman, 2010). We postulate that there is a possibility to detect a large portion of microseeping areas using the capability of spectral remote sensing to map induced mineralogical signatures. Such maps would 
then facilitate evaluating the contribution of every sedimentary basin or petroleum field in natural methane inventory.

From another perspective, the oxidation of pyrite in a sulfide-rich microseepage system may produce acid rock drainage and affect the ecosystem and the quality of drinking water in nearby areas (Swayze et al., 2000). Screening of microseepage areas by spectral remote sensing can help geologists gain a better understanding of the relative distribution of secondary minerals formed after sulfide oxidation and predict potential acid generation areas.

Unlike the natural seepage of crude oil into the marine environment, onshore seepage is not considered an important source of oil pollution. However, owing to the fact that several aspects of natural seepage are shared by anthropogenic oil spillage, any progress towards remote sensing seepage characterization can be an asset to environmental protection and vice versa.

\section{Conclusion}

Whereas oil production from offshore basins is rapidly growing, in terms of yet-to-find reserves, it has been estimated that around half of the world's total conventional oil would still come from onshore basins, of which about half is expected to be new discoveries (Schenk, 2012; 2000). The macro-, and microseepage systems associated with these unexplored (and explored) onshore accumulations have been proven to contribute substantially towards natural methane emission and global warming. Consequently, any attempt to develop methods for seepage characterization has dual implications: in one way, it can be employed as a reliable indicator for oil and gas exploration; and in the other, it can be used to disaggregate the geological emission factors in environmental assessment.

Spectral remote sensing is offering a unique opportunity to detect the full range of onshore seepage indications; typically, the VNIR-SWIR wavelengths have been used to map the alteration footprints of microseepage systems and the SWIR-LWIR wavelengths to detect the manifestation of oil and gas macroseeps. Despite the encouraging results outlined here, we believe the potentials of this state-of-the-art technology for seepage inspection is not yet fully exploited. In the case of oil seepage, this approach not only can detect the oil-shows but also has a great capacity to quantify and characterize their HC content provided that the sensor has high spatial and spectral resolutions. The emerging capability of this technique in mapping trace gas anomalies is very promising; however, it should be expanded to sense other lightweight HCs. Correspondingly, alongside methane sensing, which is particularly valuable for environmental issues, further experiments should be devised for mixed methane and ethane $\left(C_{2+}\right)$ detection, as the latter is a very useful indicator for oil and gas exploration. Overall, we need to develop additional case studies over petroliferous terrains with different oil and gas seepage properties and flow rates to evaluate the robustness of the techniques in determining the outline, content, and quantity of leakages. Such studies shall benefit from sensors available in the SWIR and LWIR wavelength ranges.

In the case of microseepage systems, the existing ambiguities in anomaly interpretation are believed to arise from several contributory factors including data restrictions, incomplete study cases, a simplistic methodology for data analysis, and above all, an immature conceptual model. In order to increase the efficiency of remote sensing approach, studies should be enriched by advanced spectral products such as the abundance and physicochemistry of minerals and supplemented by novel mineralogical indicator; an objective that is mainly achievable by hyperspectral remote sensing. Moreover, further studies should be 
directed towards a diverse range of host-rocks and geologic settings by considering the basic guidelines provided in this article aiming to give insights into the full range of secondary changes and evaluate their detectability via spectral techniques. Ultimately, a new composite microseepage model capable of accounting for the variety of secondary changes should be devised and employed in the future investigations.

\section{Acknowledgement}

We are thankful to Edward Cloutis (University of Winnipeg) and Celio Pasquini (University of Campinas) for their helpful discussion on petroleum spectroscopy. Rosa E. Pabón is acknowledged for providing the petroleum spectrum used for spectral deconvolution. We also appreciate the USGS and NASA for facilitating our access to the datasets used in the demonstration case studies. The authors would like to thank FAPESP for the research grant No. 2015/06663-7. C. R. Souza Filho thanks CNPq for research grant No. 2008$7 / 303563$.

\section{References}

Abrams, M.A., 2005. Significance of hydrocarbon seepage relative to petroleum generation and entrapment. Marine and Petroleum Geology, 22(4): 457-477.

Abrams, M.J., Conel, J.E., Lang, H.R. and Paley, H.N., 1985. The Joint NASA/Geosat Test Case Project: final report. American Association of Petroleum Geologists.

Agar, B., 1999. Spectral and mineralogical characterisation of alteration associated with hydrocarbon seepage using Geoscan AMSS MKII data over Palm Valley Thirteenth International Conference and Workshops on Applied Geologic Remote Sensing. ERIM international, Vancouver, British Columbia, Canada, pp. 110-117.

Al Shaieb, Z., Cairns, J. and Puckette, J., 1994. Hydrocarbon-induced diagenetic aureoles: Indicators of deeper, leaky reservoirs. Association of Petroleum Geochemical Explorationists Bulletin, 10: 24-48.

Almeida-Filho, R., Miranda, F.P., Galvão, L.S. and Freitas, C.C., 2002. Terrain characteristics of a tonal anomaly remotely detected in an area of hydrocarbon microseepage, Tucano Basin, north-eastern Brazil. International Journal of Remote Sensing, 23(18): 3893-3898.

Almeida-Filho, R., Miranda, F.P. and Yamakawa, T., 1999. Remote detection of a tonal anomaly in an area of hydrocarbon microseepage, Tucano basin, north-eastern Brazil. International Journal of Remote Sensing, 20(13): 2683-2688.

Aminzadeh, F., Berge, T., B., de Groot, P. and Valenti, G., 2001. Using Gas Chimneys as an Exploration Tool. World Oil, 222(5): 50-56.

Asadzadeh, S. and Souza Filho, C.R., 2016a. Investigating the capability of WorldView-3 superspectral data for direct hydrocarbon detection. Remote Sensing of Environment, 173: 162-173.

Asadzadeh, S. and Souza Filho, C.R., 2016b. A review on spectral processing methods for geological remote sensing. International Journal of Applied Earth Observation and Geoinformation, 47: 69-90.

Aske, N., Kallevik, H. and Sjöblom, J., 2001. Determination of Saturate, Aromatic, Resin, and Asphaltenic (SARA) Components in Crude Oils by Means of Infrared and Near-Infrared Spectroscopy. Energy \& Fuels, 15(5): 1304-1312.

Bailey, T.C., 1996. Application of X-band radar to sense hydrocarbon seepage. Oil and Gas Journal, 94(50): 72-75.

Bailey, T.C. and Grubb, J.M., 2006. Drilling confirms radar-mapped atmospheric seepage anomalies. Oil and Gas Journal, 104(39): 32-37.

Bailey, T.C. and Skolnik, M.I., 1996. Science behind sensing hydrocarbon seepage using X-band radar. Oil and Gas Journal, 94(49): 85-88.

Beitler, B., Chan, M.A. and Parry, W.T., 2003. Bleaching of Jurassic Navajo sandstone on Colorado Plateau Laramide highs: Evidence of exhumed hydrocarbon supergiants? Geology, 31(12): 1041-1044. 
Beitler, B., Parry, W.T. and Chan, M.A., 2005. Fingerprints of Fluid Flow: Chemical Diagenetic History of the Jurassic Navajo Sandstone, Southern Utah, U.S.A. Journal of Sedimentary Research, 75(4): 547-561.

Bell, J.H., Bowen, B.B. and Martini, B.A., 2010. Imaging spectroscopy of jarosite cement in the Jurassic Navajo Sandstone. Remote Sensing of Environment, 114(10): 2259-2270.

Boggs, S.J., 2009. Petrology of sedimentary rocks. Cambridge University Press, NY, USA, 600 pp.

Bowen, B.B., Martini, B.A., Chan, M.A. and Parry, W.T., 2007. Reflectance spectroscopic mapping of diagenetic heterogeneities and fluid-flow pathways in the Jurassic Navajo Sandstone. AAPG Bulletin, 91(2): 173-190.

Bradley, E.S., Leifer, I., Roberts, D.A., Dennison, P.E. and Washburn, L., 2011. Detection of marine methane emissions with AVIRIS band ratios. Geophysical Research Letters, 38(10): n/a-n/a.

Brown, A., 2000. Evaluation of Possible Gas Microseepage Mechanisms. AAPG Bulletin, 84(11): 1775-1789.

Calhoun, G.G., 1991. How 12 Geochemical Methods Fared in GERT Project in Permian. Oil and Gas Journal, 89(19): 62-68.

Chakraborty, S., Weindorf, D.C., Li, B., Ali Aldabaa, A.A., Ghosh, R.K., Paul, S. and Nasim Ali, M., 2015. Development of a hybrid proximal sensing method for rapid identification of petroleum contaminated soils. Science of The Total Environment, 514: 399-408.

Chakraborty, S., Weindorf, D.C., Morgan, C.L.S., Ge, Y., Galbraith, J.M., Li, B. and Kahlon, C.S., 2010. Rapid Identification of Oil-Contaminated Soils Using Visible Near-Infrared Diffuse Reflectance Spectroscopy. Journal of Environmental Quality, 39(4): 1378-1387.

Chan, M.A., Parry, W.T. and Bowman, J.R., 2000. Diagenetic Hematite and Manganese Oxides and FaultRelated Fluid Flow in Jurassic Sandstones, Southeastern Utah. AAPG Bulletin, 84(9): 1281-1310.

Chen, S., Zhao, Y., Zhao, L., Liu, Y. and Zhou, C., 2016. Hydrocarbon micro-seepage detection by altered minerals mapping from airborne hyper-spectral data in Xifeng Oilfield, China. Journal of Earth Science: 1-10.

Clark, R.N., Curchin, J.M., Hoefen, T.M. and Swayze, G.A., 2009. Reflectance spectroscopy of organic compounds: 1. Alkanes. Journal of Geophysical Research: Planets, 114(E3): 19.

Clark, R.N., Swayze, G.A., Leifer, I., Livo, K.E., Kokaly, R., Hoefen, T., Lundeen, S., Eastwood, M., Green, R.O., Pearson, N., Sarture, C., McCubbin, I., Roberts, D., Bradley, E., Steele, D., Ryan, T., Dominguez, R. and Team, a.t.A.b.V.I.I.S.A., 2010. A method for quantitative mapping of thick oil spills using imaging spectroscopy, U.S. Geological Survey Open-File Report Number 2010-1167.

Clarke, R.H. and Cleverly, R.W., 1991. Petroleum seepage and post-accumulation migration. Geological Society, London, Special Publications, 59(1): 265-271.

Cloutis, E.A., 1989. Spectral reflectance properties of hydrocarbons: Remote-sensing implications. Science, 245(4914): 165-168.

Cloutis, E.A., 1990. Identification, detection and characterization of individual tar sand phases using diffuse reflectance spectroscopy (0.35-2.6 $\mu \mathrm{m})$. AOSTRA Journal of Research, 6: 17-27.

Cloutis, E.A., Gaffey, M.J. and Moslow, T.F., 1995. Characterization of minerals in oil sands by reflectance spectroscopy. 74(6): 874-879.

Coates, J., 2006. Interpretation of Infrared Spectra, A Practical Approach, Encyclopedia of Analytical Chemistry. John Wiley \& Sons, Ltd, pp. 1-23.

Connolly, D., Aminzadeh, F., Brouwer, F. and Nielsen, S., 2013. Detection of Subsurface Hydrocarbon Seepage in Seismic Data: Implications for Charge, Seal, Overpressure, and Gas-hydrate Assessment. In: F. Aminzadeh, T.B. Berge and D.L. Connolly (Editors), Hydrocarbon Seepage: From source to surface. SEG \& AAPG, Tulsa, USA, pp. 199-220.

Correa Pabón, R.E. and Souza Filho, C.R., 2016. Spectroscopic characterization of red latosols contaminated by petroleum-hydrocarbon and empirical model to estimate pollutant content and type. Remote Sensing of Environment, 175: 323-336.

Crowley, J.K., Williams, D.E., Hammarstrom, J.M., Piatak, N., Chou, I.-M. and Mars, J.C., 2003. Spectral reflectance properties $(0.4-2.5 \mu \mathrm{m})$ of secondary Fe-oxide, Fe-hydroxide, and Fe-sulphate-hydrate minerals associated with sulphide-bearing mine wastes. Geochemistry: Exploration, Environment, Analysis, 3(3): 219-228. 
Curto, J.B., Pires, A.C.B., Moreira, A.S. and Crósta, Á.P., 2011. Strategies for detecting hydrocarbon microseepages using airborne geophysics integrated with remote sensing data, 12th International Congress of the Brazilian Geophysical Society Brazilian Geophysical Society, Rio de Janeiro, Brazil, pp. 1-6.

Davidson, M.J., 2004. Evolution of scientific surface geochemical exploration. Oil and Gas Journal, 102(23): 35-38.

Donovan, T.J., 1974. Petroleum Microseepage at Cement, Oklahoma; Evidence and Mechanism. AAPG Bulletin, 58(3): 429-446.

Donovan, T.J., Friedman, I. and Gleason, J.D., 1974. Recognition of Petroleum-bearing Traps by Unusual Isotopic Compositions of Carbonate-cemented Surface Rocks. Geology, 2(7): 351-354.

Donovan, T.J., Noble, R.L., friedman, I. and Gleason, J.D., 1975. A possible petroleum related geochemical anomaly in surface rocks, USGS, Open-File Report No 75-47

Donovan, T.J., Roberts, A.A. and Dalziel, M.C., 1979-1982. Epigenetic zoning in surface and near-surface rocks resulting from seepage-induced redox gradients, Velma oil field, Oklahoma: A synopsis. The Shale Shaker Digest XX, XXX-XXXII: 175-181.

Duchscherer, J.W., 1980. Geochemical methods of prospecting for hydrocarbons. Oil and Gas Journal, DEC: 194-208.

Duchscherer, J.W., 1982. Geochemical exploration for hydrocarbons, no new tricks - but an old dog. Oil and Gas Journal, July: 163-176.

Eichhubl, P., Taylor, W.L., Pollard, D.D. and Aydin, A., 2004. Paleo-fluid flow and deformation in the Aztec Sandstone at the Valley of Fire, Nevada-Evidence for the coupling of hydrogeologic, diagenetic, and tectonic processes. Geological Society of America Bulletin, 116(9-10): 1120-1136.

Ellis, J.M., Davis, H.H. and Zamudio, J.A., 2001. Exploring for onshore oil seepas with hyperspectral imaging. Oil and Gas Journal, 99(37): 49-58.

Ellwood, B.B. and Burkart, B., 1996. Test of hydrocarbon-induced magnetic patterns in soils: the sanitary landfill as laboratory. In: D. Schumacher and M.A. Abrams (Editors), Hydrocarbon migration and its near-surface expresseion. AAPG Memoir 66, Tulsa, OK, U.S.A., pp. 91-98.

Elwood Madden, M.E., Bodnar, R.J. and Rimstidt, J.D., 2004. Jarosite as an indicator of water-limited chemical weathering on Mars. Nature, 431(7010): 821-823.

Etiope, G., 2009. GLOGOS, A new global onshore gas-oil seeps dataset, Search and discovery, Article \#70071. AAPG online journal.

Etiope, G., 2015. Natural Gas Seepage: The Earth's Hydrocarbon Degassing. Springer International Publishing, Switzerland.

Etiope, G. and Ciccioli, P., 2009. Earth's Degassing: A Missing Ethane and Propane Source. Science, 323(5913): 478-478.

Etiope, G. and Klusman, R.W., 2010. Microseepage in drylands: Flux and implications in the global atmospheric source/sink budget of methane. Global and Planetary Change, 72(4): 265-274.

Etiope, G., Lassey, K.R., Klusman, R.W. and Boschi, E., 2008. Reappraisal of the fossil methane budget and related emission from geologic sources. Geophysical Research Letters, 35(9): L09307.

Etiope, G. and Martinelli, G., 2002. Migration of carrier and trace gases in the geosphere: an overview. Physics of the Earth and Planetary Interiors, 129(3-4): 185-204.

Eventov, L., 2000. The nature and interpretation of geophysical and geochemical anomalies over oil and gas fields. The Leading Edge, 19(5): 488-490.

Everett, J.R., Staskowski, R.J. and Jengo, C., 2002. Remote sensing and GIS enable future exploration success. World Oil, 223(11): 59-63.

Feder, A.M., 1985. Contemporary Remote Sensing for Hydrocarbon Exploration and Development-with Selected Case Histories. Oil and Gas Journal.

Fingas, M. and Brown, C.E., 2014. Oil Spill Remote Sensing, Handbook of Oil Spill Science and Technology. John Wiley \& Sons, Inc, pp. 311-356.

Foote, R.S., 1992. Use of Magnetic Field Aids Oil Search. Oil and Gas Journal, 90(18): 137-142. 
Foote, R.S., 1996. Relationship of near-surface magnetic anomalies to oil- and gas producing areas. In: D. Schumacher and M.A. Abrams (Editors), Hydrocarbon migration and its near-surface expresseion. AAPG Memoir 66, Tulsa, OK, U.S.A., pp. 111-126.

Foote, R.S., 2013. Value of MSRI measurement of Selma exploration drill cuttings. Oil and Gas Journal, 111(10): 66-73.

Frankenberg, C., Thorpe, A.K., Thompson, D.R., Hulley, G., Kort, E.A., Vance, N., Borchardt, J., Krings, T., Gerilowski, K., Sweeney, C., Conley, S., Bue, B.D., Aubrey, A.D., Hook, S. and Green, R.O., 2016. Airborne methane remote measurements reveal heavy-tail flux distribution in Four Corners region. Proceedings of the National Academy of Sciences, 113(35): 9734-9739.

Frassy, F., Maianti, P., Marchesi, A., Nodari, F.R., Dalla Via, G., De Paulis, R., Biffi, P.G. and Gianinetto, M., 2015. Satellite remote sensing for hydrocarbon exploration in new venture areas, 2015 IEEE International Geoscience and Remote Sensing Symposium (IGARSS), pp. 2884-2887.

Freeman, H., 2003. Evaluation of the use of hyperspectral imagery for identification of microseeps near Santa Barbara, California, West Virginia University, $22 \mathrm{pp}$.

Fu, B., Zheng, G., Ninomiya, Y., Wang, C. and Sun, G., 2007. Mapping hydrocarbon-induced mineralogical alteration in the northern Tian Shan using ASTER multispectral data. Terra Nova, 19(4): 225-231.

Gaffey, S.J., 1987. Spectral reflectance of carbonate minerals in the visible and near infrared (0.35-2.55 um): Anhydrous carbonate minerals. Journal of Geophysical Research: Solid Earth, 92(B2): 1429-1440.

Garden, I.R., Guscott, S.C., Burley, S.D., Foxford, K.A., Walsh, J.J. and Marshall, J., 2001. An exhumed palaeohydrocarbon migration fairway in a faulted carrier system, Entrada Sandstone of SE Utah, USA. Geofluids, 1(3): 195-213.

Gorenc, M.A. and Chan, M.A., 2015. Hydrocarbon-induced diagenetic alteration of the Permian White Rim Sandstone, Elaterite Basin, southeast Utah. AAPG Bulletin, 99(5): 807-829.

Gutierrez, C.I., Tan, S.S. and Clahan, K.B., 2008. Geologic map of the east half Santa Barbara $30^{\prime} \times 60^{\prime}$ Quadrangle, California. California Geological Survey.

Holysh, S. and Toth, J., 1996. Flow of formation waters: Likely cause for poor definition of soil gas anomalies over oil fields in east-central Alberta. In: D. Schumacher and M.A. Abrams (Editors), Hydrocarbon migration and its near-surface expression. AAPG Memoir 66, Tulsa, OK, U.S.A., pp. 255-277.

Hörig, B., Kühn, F., Oschütz, F. and Lehmann, F., 2001. HyMap hyperspectral remote sensing to detect hydrocarbons. International Journal of Remote Sensing, 22(8): 1413-1422.

Horvitz, L., 1980. Near-surface evidence of hydrocarbon movement from depth, SG 10: Problems of Petroleum Migration. AAPG, pp. 241-269.

Horvitz, L., 1985. Geochemical Exploration for Petroleum. Science, 229(4716): 821-827.

Hulley, G.C., Duren, R.M., Hopkins, F.M., Hook, S.J., Vance, N., Guillevic, P., Johnson, W.R., Eng, B.T., Mihaly, J.M., Jovanovic, V.M., Chazanoff, S.L., Staniszewski, Z.K., Kuai, L., Worden, J., Frankenberg, C., Rivera, G., Aubrey, A.D., Miller, C.E., Malakar, N.K., Sánchez Tomás, J.M. and Holmes, K.T., 2016. High spatial resolution imaging of methane and other trace gases with the airborne Hyperspectral Thermal Emission Spectrometer (HyTES). Atmos. Meas. Tech., 9(5): 2393-2408.

Hunt, G.R., 1977. Spectral signatures of particulate minerals in the visible and near infrared. Geophysics, 42(3): 501-513.

Hunt, J.M., 1996. Petroleum geochemistry and geology. W. H. Freeman, New York.

Jengo, C.M. and Vincent, R.K., 1999. Hyperspectral imaging of ancient hydrocarbon seeps, Wind River basin, Wyoming, Thirteenth International Conference and Workshops on Applied Geologic Remote Sensing. ERIM international, Vancouver, British Columbia, Canada, pp. 118-125.

Jones, V.T. and Drozd, R.J., 1983. Predictions of oil or gas potential by near-surface geochemistry. AAPG Bulletin, 67(6): 932-952.

Khan, S.D. and Jacobson, S., 2008. Remote sensing and geochemistry for detecting hydrocarbon microseepages. Geological Society of America Bulletin, 120(1-2): 96-105.

Kirkland, D.W., Denison, R.E. and Rooney, M.A., 1995. Diagenetic alteration of Permian strata at oil fields of south central Oklahoma, USA. Marine and Petroleum Geology, 12(6): 629-644. 
Klusman, R.M., 2002. Interpretation and display of surface geochemical data. In: D. Schumacher and L.A. LeSchack (Editors), Surface exploration case histories: Application of geochemistry, magnetics, and remote sensing. AAPG and SEG, Tulsa, OK, U.S.A., pp. 1-24.

Klusman, R.M. and Saeed, M.A., 1996. Comparison of light hydrocarbon microseepage mechanisms. In: D. Schumacher and M.A. Abrams (Editors), Hydrocarbon migration and its near-surface expresseion. AAPG Memoir 66, Tulsa, OK, U.S.A., pp. 157-168.

Kokaly, R.F., Couvillion, B.R., Holloway, J.M., Roberts, D.A., Ustin, S.L., Peterson, S.H., Khanna, S. and Piazza, S.C., 2013. Spectroscopic remote sensing of the distribution and persistence of oil from the Deepwater Horizon spill in Barataria Bay marshes. Remote Sensing of Environment, 129(0): 210-230.

Kühn, F., Oppermann, K. and Hörig, B., 2004. Hydrocarbon Index - an algorithm for hyperspectral detection of hydrocarbons. International Journal of Remote Sensing, 25(12): 2467-2473.

Lammoglia, T. and Souza Filho, C.R., 2011. Spectroscopic characterization of oils yielded from Brazilian offshore basins: Potential applications of remote sensing. Remote Sensing of Environment, 115(10): 2525-2535.

Lammoglia, T. and Souza Filho, C.R., 2012. Mapping and characterization of the API gravity of offshore hydrocarbon seepages using multispectral ASTER data. Remote Sensing of Environment, 123(0): $381-$ 389.

Lammoglia, T., Souza Filho, C.R. and Filho, R.A., 2008. Characterization of hydrocarbon microseepages in the Tucano basin, (Brazil) through hyperspectral classification and neural network Analysis of Advanced Spaceborne Thermal Emission and Reflection Radiometer (ASTER) data, The International Archives of the Photogrammetry, Remote Sensing and Spatial Information Sciences, Beijing, pp. 1195-1200.

Land, J.P., 1996. Nonseismic methods can provide many views of a drillsite. Oil and Gas Journal, 94(9): 69-73.

Leifer, I., Lehr, W.J., Simecek-Beatty, D., Bradley, E., Clark, R., Dennison, P., Hu, Y., Matheson, S., Jones, C.E., Holt, B., Reif, M., Roberts, D.A., Svejkovsky, J., Swayze, G. and Wozencraft, J., 2012a. State of the art satellite and airborne marine oil spill remote sensing: Application to the BP Deepwater Horizon oil spill. Remote Sensing of Environment, 124(0): 185-209.

Leifer, I., Tratt, D.M., Realmuto, V.J., Gerilowski, K. and Burrows, J.P., 2012b. Remote sensing atmospheric trace gases with infrared imaging spectroscopy. Eos, Transactions American Geophysical Union, 93(50): 525-525.

LeSchack, L.A., 1997. Results of magnetic HGI and radiometric surveys in W. Canada. Oil and Gas Journal, 95(20): 84-89.

LeSchack, L.A. and Van Alstine, D.R., 2002. High-resolution ground-magnetic (HRGM) and radiometric surveys for hydrocarbon exploration: Six case histories in Western Canada. In: D. Schumacher and L.A. LeSchack (Editors), Surface exploration case histories: Application of geochemistry, magnetics, and remote sensing. AAPG and SEG, Tulsa, OK, U.S.A., pp. 67-156.

Lilburn, R.A. and Zuhair, A.-S., 1984. Geochemistry and isotopic composition of Hydrocarbon-Induced Diagenetic Aureole (HIDA), Cement Field Oklahoma. The Shale Shaker Digest XI, XXXIII-XXXV: 46-72.

Link, W.K., 1952. Significance of oil and gas seeps in world oil exploration. AAPG Bulletin, 36(8): 1505-1540.

Lyder, D., Feng, J., Rivard, B., Gallie, A. and Cloutis, E., 2010. Remote bitumen content estimation of Athabasca oil sand from hyperspectral infrared reflectance spectra using Gaussian singlets and derivative of Gaussian wavelets. Fuel, 89(3): 760-767.

Macgregor, D.S., 1993. Relationships between seepage, tectonics and subsurface petroleum reserves. Marine and Petroleum Geology, 10(6): 606-619.

Magoon, L.B. and Beaumont, E.A., 1999. Petroleum Systems. In: E.A. Beaumont and N.H. Foster (Editors), Treatise of Petroleum Geology / Handbook of Petroleum Geology: Exploring for Oil and Gas Traps. AAPG, pp. 3-1 - 3-34.

Malhotra, R.V., Birnie, R.W. and Johnson, B.G., 1989. Detection of surficial changes associated with hydrocarbon seepage Sheep Mountain anticline, Bighorn basin, Wyoming, Seventh Thematic Conference on Remote Sensing for Exploration Geology. ERIM international, Calgarly, Alberta, Canada, pp. 1097-1110. 
Marrs, R.W. and Paylor., E.D., 1987. Investigation of a surface spectral anomaly at Table Rock gas field, Wyoming. GEOPHYSICS, 52(7): 841-857.

Matthews, M.D., 1986. Effects of hydrocarbon leakage on earth surface materials. In: M.J. Davidson (Editor), Unconventional methods in prospecting for petroleum and natural gas IV. outhern Methodist University Press, Institute for the Study of Earth and Man, Dallas, pp. 27-44.

McCoy, R.M., Blake, J.G. and Andrews, K.L., 2001. Detecting hydrocarbon microseepage using hydrocarbon absorption bands of reflectance spectra of surface soils. Oil and Gas Journal, 99(22): 40-45.

Nielsen, G.B., Chan, M.A. and Petersen, E.U., 2009. Diagenetic coloration facies and alteration history of the jurassic Navajo sandstone, Zion national park and vicinity, Southwestern Utah, CD-ROM.

Nielson, G.B., Chan, M.A. and Bowen, B., 2014. Iron-rich horizons in the Jurassic Navajo Sandstone, southwestern Utah: progressive cementation and permeability inversion. In: J.S. MacLean, R.F. Biek and J.E. Huntoon (Editors), Geology of Utah's Far South. Utah Geological Association Publication, pp. 215-236.

Okparanma, R.N., Coulon, F. and Mouazen, A.M., 2014. Analysis of petroleum-contaminated soils by diffuse reflectance spectroscopy and sequential ultrasonic solvent extraction-gas chromatography. Environmental Pollution, 184: 298-305.

Parry, W.T., Chan, M.A. and Beitler, B., 2004. Chemical bleaching indicates episodes of fluid flow in deformation bands in sandstone. AAPG Bulletin, 88(2): 175-191.

Parry, W.T., Chan, M.A. and Nash, B.P., 2009. Diagenetic characteristics of the Jurassic Navajo Sandstone in the Covenant oil field, central Utah thrust belt. AAPG Bulletin, 93(8): 1039-1061.

Perry, A.F., 2006. Unconventional exploration technologies: Take another look. World Oil, 227(9): 45-52.

Perry, S.L. and Kruse, F.A., 2010. Evidence of hydrocarbon seepage using multispectral satellite imagery, Kurdistan, Iraq, AAPG International Convention and Exhibition, AAPG Search and Discovery Article \#90108, Calgary, Alberta, Canada, pp. 7.

Petrovic, A., Khan, S.D. and Chafetz, H.S., 2008. Remote detection and geochemical studies for finding hydrocarbon-induced alterations in Lisbon Valley, Utah. Marine and Petroleum Geology, 25(8): 696705.

Petrovic, A., Khan, S.D. and Thurmond, A.K., 2012. Integrated hyperspectral remote sensing, geochemical and isotopic studies for understanding hydrocarbon-induced rock alterations. Marine and Petroleum Geology, 35(1): 292-308.

Pirajno, F., 2009. Hydrothermal Processes and Mineral Systems. Springer Science.

Potter-McIntyre, S., Allen, J., Lee, S.Y., Han, W.S., Chan, M. and McPherson, B., 2013. Iron precipitation in a natural CO2 reservoir: Jurassic Navajo Sandstone in the northern San Rafael Swell, UT, USA. Geofluids, 13(1): 82-92.

Potter II, R.W., Harrington, P.A., Silliman, A.H. and Viellena, J.H., 1996. Significance of Geochemical Anomalies in Hydrocarbon Exploration: One Company's Experience. In: D. Schumacher and M.A. Abrams (Editors), Hydrocarbon migration and its near-surface expression. AAPG Memoir 66, Tulsa, OK, U.S.A., pp. 431-440.

Prelat, A., Gunaratne, S., Huebner, L., Freeman, C., Cook, A. and Soriano, C., 2013. Airborne hyperspectral detection of natural offshore and onshore hydrocarbon seeps. In: F. Aminzadeh, T.B. Berge and D.L. Connolly (Editors), Hydrocarbon Seepage: From Source to Surface. American Association of Petroleum Geologists, Tulsa, OK, U.S.A., pp. 171-182.

Price, L.C., 1986. A critical review and proposed working model of surface geochemical exploration. In: M.J. Davidson (Editor), Unconventional methods in exploration for petroleum and natural gas. Southern Methodist University Press, Dallas, pp. 245-304.

Price, L.C., 1996. Research-derived insights into surface geochemical hydrocarbon exploration. In: D. Schumacher and M.A. Abrams (Editors), Hydrocarbon migration and its near-surface expression. AAPG Memoir 66, Tulsa, OK, U.S.A., pp. 285-307.

Prokopovich, N.P., Cole, R.C. and Nishi, C.K., 1971. Alteration of sediments by natural gases in western Merced County, California. AAPG Bulletin, 55(6): 826-832. 
Qin, Q., Zhang, Z., Chen, L., Wang, N. and Zhang, C., 2016. Oil and gas reservoir exploration based on hyperspectral remote sensing and super-low-frequency electromagnetic detection. Journal of Applied Remote Sensing, 10(1): 016017-016017.

Rainoldi, A.L., Franchini, M., Beaufort, D., Patrier, P., Giusiano, A., Impiccini, A. and Pons, J., 2014. Large-Scale Bleaching of Red Beds Related To Upward Migration of Hydrocarbons: Los Chihuidos High, Neuquén Basin, Argentina. Journal of Sedimentary Research, 84(5): 373-393.

Rice, G.K., Belt Jr, J.Q. and Berg, G.E., 2002. Soil-gas Hydrocarbon Pattern Changes During a West Texas Waterflood. In: D. Schumacher and L.A. LeSchack (Editors), Surface exploration case histories: Application of geochemistry, magnetics, and remote sensing. AAPG and SEG, Tulsa, OK, U.S.A., pp. 157-174.

Rice, G.K., King, G. and Henson, J., 2016. Integration of geology, seismic, and geochemical data - Theory and practice in Cheeseburger Field, Stonewall County, Texas, USA. Interpretation, 4(2): T215-T225.

Richers, D.M., Jones, V.T., Matthews, M.D., Maciolek, J., Pirkle, R.J. and Sidle, W.C., 1986. The 1983 Landsat soil-gas geochemical survey of Patrick Draw area, Sweetwater County, Wyoming. AAPG Bulletin, 70(7): 869-887.

Richers, D.M., Reed, R.J., Horstman, K.C., Michels, G.D., Baker, R.N., Lundell, L. and Marrs, R.W., 1982. Landsat and soil-gas geochemical study of Patrick Draw oil field, Sweetwater County, Wyoming. AAPG Bulletin, 66(7): 903-922.

Roberts, D.A., Bradley, E.S., Cheung, R., Leifer, I., Dennison, P.E. and Margolis, J.S., 2010. Mapping methane emissions from a marine geological seep source using imaging spectrometry. Remote Sensing of Environment, 114(3): 592-606.

Salati, S., van Ruitenbeek, F., van der Meer, F. and Naimi, B., 2014a. Detection of alteration induced by onshore gas seeps from ASTER and WorldView-2 data. Remote Sensing, 6(4): 3188-3209.

Salati, S., van Ruitenbeek, F.J.A., de Smeth, J.B. and van der Meer, F.D., 2014b. Spectral and geochemical characterization of onshore hydrocarbon seep-induced alteration in the Dezful embayment, southwest Iran. AAPG Bulletin, 98(9): 1837-1857.

Saunders, D.F., Burson, K.R., Branch, J.F. and Thompson, C.K., 1993a. New method of aerial and surface radiometric prospecting for oil, gas. Oil and Gas Journal, 91(38): 104-109.

Saunders, D.F., Burson, K.R., Branch, J.F. and Thompson, C.K., 1993b. Relation of thorium-normalized surface and aerial radiometric data to subsurface petroleum accumulations. Geophysics, 58(10): 1417-1427.

Saunders, D.F., Burson, K.R. and Thompson, C.K., 1991. Observed relation of soil magnetic susceptibility and soil gas hydrocarbon analyses to subsurface petroleum accumulations. AAPG Bulletin, 75(3): 389408.

Saunders, D.F., Burson, K.R. and Thompson, C.K., 1999. Model for hydrocarbon microseepage and related near-surface alterations. AAPG Bulletin, 83(1): 170-185.

Saunders, D.F. and Terry, S.A., 1985. Onshore exploration using new geochemistry and geomorphology. Oil and Gas Journal, September, 16(38): 126-130.

Scafutto, R.D.P.M., 2015. Sensoriamento remoto hiperespectral aplicado à identificação remota de solos e rochas impregnados com hidrocarbonetos, University of Campinas, Campinas, Brazil, 258 pp.

Scafutto, R.D.P.M. and Souza Filho, C.R., 2016. Quantitative characterization of crude oils and fuels in mineral substrates using reflectance spectroscopy: Implications for remote sensing. International Journal of Applied Earth Observation and Geoinformation, 50: 221-242.

Scafutto, R.D.P.M., Souza Filho, C.R. and Rivard, B., 2016. Characterization of mineral substrates impregnated with crude oils using proximal infrared hyperspectral imaging. Remote Sensing of Environment, 179: 116-130.

Schenk, C.J., 2012. An estimate of undiscovered conventional oil and gas resources of the world, 2012.

Scholte, K.H., 2005. Hyperspectral remote sensing and mud volcanism in azerbaijan (PhD thesis), Delft University of Technology, Netherlands, $147 \mathrm{pp}$.

Scholte, K.H., Hommels, A., Munoz-Sabater, J., Hanssen, R.F., Van der Meer, F.D., Kroonenberg, S.B., Aliyeva, E., Huseynov, D. and Guliev, I., 2003. Preliminary ASTER and InSAR imagery combination for mud 
volcano dynamics, Azerbaijan, Geoscience and Remote Sensing Symposium, 2003. IGARSS '03. Proceedings. 2003 IEEE International, pp. 1573-1575.

Schumacher, D., 1996. Hydrocarbon-induced alteration of soils and sediments. In: D. Schumacher and M.A. Abrams (Editors), Hydrocarbon migration and its near-surface expression. AAPG Memoir 66, Tulsa, OK, U.S.A., pp. 71-89.

Schumacher, D., 1999. Surface Geochemical Exploration for Petroleum. In: E.A. Beaumont and N.H. Foster (Editors), Treatise of Petroleum Geology / Handbook of Petroleum Geology: Exploring for Oil and Gas Traps. AAPG, pp. 18-1 - 18-27.

Schumacher, D., 2010. Integrating hydrocarbon microseepage data with seismic data doubles exploration success, 34th annual conference and exhibition. Proceedings, Indonesian petroleum association, Indonesia, pp. 11.

Schumacher, D.D., 2000. Surface geochemical exploration for oil and gas: New life for an old technology. The Leading Edge, 19(3): 258-261.

Schwartz, G., Ben-Dor, E. and Eshel, G., 2013. Quantitative Assessment of Hydrocarbon Contamination in Soil Using Reflectance Spectroscopy: A “Multipath" Approach. Applied Spectroscopy, 67(11): 1323-1331.

Schwietzke, S., Sherwood, O.A., Bruhwiler, L.M.P., Miller, J.B., Etiope, G., Dlugokencky, E.J., Michel, S.E., Arling, V.A., Vaughn, B.H., White, J.W.C. and Tans, P.P., 2016. Upward revision of global fossil fuel methane emissions based on isotope database. Nature, 538(7623): 88-91.

Segal, D.B. and Merin, I.S., 1989. Successful use of Landsat Thematic Mapper data for mapping hydrocarbon microseepage-induced mineralogic alteration, Lisbon Valley, Utah. Photogrammetric Engineering and Remote Sensing, 55(8): 1137-1145.

Segal, D.B., Ruth, M.D. and Merin, I.S., 1986. Remote detection of anomalous mineralogy associated with hydrocarbon production, Lisbon Valley, Utah. The Moutain Geologist, 23(2): 51-62.

Shaw, R.C. and Kratochvil, B., 1990. Near-infrared diffuse reflectance analysis of Athabasca oil sand. Analytical Chemistry, 62(2): 167-174.

Shi, P., Fu, B., Ninomiya, Y., Sun, J. and Li, Y., 2012. Multispectral remote sensing mapping for hydrocarbon seepage-induced lithologic anomalies in the Kuqa foreland basin, south Tian Shan. Journal of Asian Earth Sciences, 46(0): 70-77.

Short, N.M., 1977. Exploration for fossil and nuclear fuels from orbital altitudes. In: W.L. Smith (Editor), Remote-sensing applications for mineral exploration. Dowden, Hutchinson, and Ross, Pennsylvania.

Simpson, C.J., 1978. LANDSAT: developing techniques and applications in mineral and petroleum exploration BMR Journal of Australian Geology \& Geophysics, 3: 181-191.

Simpson, C.J., Wilford, J.R., Macias, L.F. and Korsch, R.J., 1991. Remote sensing of petroleum gas seepage alteration Palm Valley Gas Field, Amadeus Basin, central Australia., Proceedings of the Eighth Thematic Conference on Geologic Remote Sensing. ERIM, Denver, Colorado, USA, pp. 1463-1472.

Smailbegovic, A., Gray, K., Johnson, K., Murphy, V. and Holbrook, R., 2009. Spectroscopic and hyperspectral evaluation of possible hydrocarbon occurrences in estuarine sediments, North Charleston, South Carolina, USA, 2009 First Workshop on Hyperspectral Image and Signal Processing: Evolution in Remote Sensing, pp. 1-5.

Souza Filho, C.R., Augusto, V., Oliveira, W.J. and Lammoglia, T., 2008. Detecção de exsudações de hidrocarbonetos por geobotânica e sensoriamento remoto multi-temporal: estudo de caso no Remanso do Fogo (MG). Revista Brasileira de Geociências, 38(2): 228-243.

Stallard, B.R., Garcia, M.J. and Kaushik, S., 1996. Near-IR Reflectance Spectroscopy for the Determination of Motor Oil Contamination in Sandy Loam. Applied Spectroscopy, 50(3): 334-338.

Staskowski, R.J., Jengo, C. and Everett, J.R., 2004. Utility of ASTER for detecting hydrocarbon [Abstract], American Association of Petroleum Geologists Annual Convention, Dallas, Texas, pp. 6.

Sunshine, J.M., Pieters, C.M. and Pratt, S.F., 1990. Deconvolution of mineral absorption bands: an improved approach. Journal of Geophysical Research, 93: 6955-6966.

Swayze, G.A., Smith, K.S., Clark, R.N., Sutley, S.J., Pearson, R.M., Vance, J.S., Hageman, P.L., Briggs, P.H., Meier, A.L., Singleton, M.J. and Roth, S., 2000. Using Imaging Spectroscopy To Map Acidic Mine Waste. Environmental Science \& Technology, 34(1): 47-54. 
Tangestani, M.H. and Validabadi, K., 2014. Mineralogy and geochemistry of alteration induced by hydrocarbon seepage in an evaporite formation; a case study from the Zagros Fold Belt, SW Iran. Applied Geochemistry, 41: 189-195.

Tedesco, S.A., 1995. Surface geochemistry in petroleum exploration. Chapman and Hall, Inc, New York. Tedesco, S.A., 1999. Anomaly shifts indicate rapid surface seep rates. Oil and Gas Journal, 97(13): 69-72.

Thompson, C.K., Saunders, D.F. and Burson, K.R., 1994. Model advanced for hydrocarbon microseepage, related alterations. Oil and Gas Journal, 92(46): 95-99.

Thorpe, A.K., Frankenberg, C., Aubrey, A.D., Roberts, D.A., Nottrott, A.A., Rahn, T.A., Sauer, J.A., Dubey, M.K., Costigan, K.R., Arata, C., Steffke, A.M., Hills, S., Haselwimmer, C., Charlesworth, D., Funk, C.C., Green, R.O., Lundeen, S.R., Boardman, J.W., Eastwood, M.L., Sarture, C.M., Nolte, S.H., McCubbin, I.B., Thompson, D.R. and McFadden, J.P., 2016. Mapping methane concentrations from a controlled release experiment using the next generation airborne visible/infrared imaging spectrometer (AVIRIS-NG). Remote Sensing of Environment, 179: 104-115.

Thorpe, A.K., Frankenberg, C. and Roberts, D.A., 2014. Retrieval techniques for airborne imaging of methane concentrations using high spatial and moderate spectral resolution: application to AVIRIS. Atmos. Meas. Tech., 7(2): 491-506.

Thorpe, A.K., Roberts, D.A., Bradley, E.S., Funk, C.C., Dennison, P.E. and Leifer, I., 2013. High resolution mapping of methane emissions from marine and terrestrial sources using a Cluster-Tuned Matched Filter technique and imaging spectrometry. Remote Sensing of Environment, 134: 305-318.

Thrasher, J., Fleet, A.J., Hay, S.J., Hovland, M. and Düppenbecker, S., 1996. Understanding Geology as the key to using seepage in exploration: The spectrum of seepage styles. In: D. Schumacher and M.A. Abrams (Editors), Hydrocarbon migration and its near-surface expression. AAPG Memoir 66, Tulsa, OK, U.S.A., pp. 223-241.

Tratt, D.M., Buckland, K.N., Hall, J.L., Johnson, P.D., Keim, E.R., Leifer, I., Westberg, K. and Young, S.J., 2014. Airborne visualization and quantification of discrete methane sources in the environment. Remote Sensing of Environment, 154: 74-88.

USGS, 2000. U.S. Geological Survey World Petroleum Assessment 2000: Description and Results. 60.

van Der Meer, F., van Dijk, P., van Der Werff, H. and Yang, H., 2002. Remote sensing and petroleum seepage: a review and case study. Terra Nova, 14(1): 1-17.

van der Werff, H., 2006. Knowledge based remote sensing of complex objects recognition of spectral and spatial patterns resulting from natural hydrocarbon, Enschede, The Netherlands, $138 \mathrm{pp}$.

van der Werff, H.M.A., Bakker, W.H., van der Meer, F.D. and Siderius, W., 2006. Combining spectral signals and spatial patterns using multiple Hough transforms: An application for detection of natural gas seepages. Computers \& Geosciences, 32(9): 1334-1343.

Wang, Y. and Ding, X., 2000. Hydrocarbon Alteration Characteristics of Soils and Mechanism for Detection by Remote Sensing in East Sichuan Area, China. Natural Resources Research, 9(4): 295-305.

Warren, R.K., 2012. Near-surface resistivity for hydrocarbon detection. The Leading Edge, 31(10): 1182-1191.

Watanabe, H., Hayashi, K., Saeki, T., Maksyutov, S., Nasuno, I., Shimono, Y., Hirose, Y., Takaichi, K., Kanekon, S., Ajiro, M., Matsumoto, Y. and Yokota, T., 2015. Global mapping of greenhouse gases retrieved from GOSAT Level 2 products by using a kriging method. International Journal of Remote Sensing, 36(6): 1509-1528.

Weibel, R., 1998. Diagenesis in oxidising and locally reducing conditions - an example from the Triassic Skagerrak Formation, Denmark. Sedimentary Geology, 121(3-4): 259-276.

Wigley, M., Kampman, N., Dubacq, B. and Bickle, M., 2012. Fluid-mineral reactions and trace metal mobilization in an exhumed natural CO2 reservoir, Green River, Utah. Geology, 40(6): 555-558.

Winkelmann, K.H., 2005. On the applicability of imaging spectrometry for the detection and investigation of contaminated sites with particular consideration given to the detection of fuel hydrocarbon contaminants in soil, Brandenburg University of Technology, Brandenburg, Germany, $236 \mathrm{pp}$.

Wolleben, J.A. and Greenlee, D.W., 2002. Successful application of micromagnetic data to focus hydrocarbon exploration. In: D. Schumacher and L.A. LeSchack (Editors), Surface exploration case histories: 
Application of geochemistry, magnetics, and remote sensing. AAPG and SEG, Tulsa, OK, U.S.A., pp. 175-191.

Worden, R.H., Smalley, P.C. and Fallick, A.E., 1997. Sulfur cycle in buried evaporites. Geology, 25(7): 643-646.

Xuejing, X., 1992. Local and regional surface geochemical exploration for oil and gas. Journal of Geochemical Exploration, 43(1): 25-42.

Yang, H., Meer, F.V.D., Zhang, J. and Kroonenberg, S.B., 2000. Direct detection of onshore hydrocarbon microseepages by remote sensing techniques. Remote Sensing Reviews, 18(1): 1-18.

Yergin, D., 1992. The Prize: The Epic Quest for Oil, Money, and Power. Simon \& Schuster.

Zhang, G., Zou, L., Shen, X., Lu, S., Li, C. and Chen, H., 2009. Remote sensing detection of heavy oil through spectral enhancement techniques in the western slope zone of Songliao Basin, China. AAPG Bulletin, 93(1): 31-49.

Zheng, G., Fu, B., Takahashi, Y., Kuno, A., Matsuo, M. and Zhang, J., 2010. Chemical speciation of redox sensitive elements during hydrocarbon leaching in the Junggar Basin, Northwest China. Journal of Asian Earth Sciences, 39(6): 713-723. 